\title{
Influence of the Linker Length on the Cytotoxicity of Homobinuclear Ruthenium(II) and Gold(I) Complexes
}

\author{
Lucinda K. Batchelor, Emilia Păunescu, Mylène Soudani, Rosario Scopelliti, and Paul J. Dyson*(i) \\ Institut des Sciences et Ingénierie Chimiques, Ecole Polytechnique Fédérale de Lausanne (EPFL), CH-1015 Lausanne, Switzerland
}

Supporting Information

ABSTRACT: Dinuclear metal complexes have emerged as a promising class of anticancer compounds with the ability to cross-link biomolecular targets. Here, we describe two novel series of phosphine-linked dinuclear ruthenium(II) $p$-cymene and gold(I) complexes, in which the length of the connecting poly(ethylene glycol) chain has been systematically modified. The impact of the multinuclearity, lipophilicity, and linker length on the antiproliferative activity of the compounds on tumorigenic (A2780 and A2780cisR) and nontumorigenic (HEK-293) cell lines was assessed. The dinuclear ruthenium(II) complexes were considerably more cytotoxic than their mononuclear counterparts, and a correlation between the lipophilicity of the linker and the cytotoxicity was observed, whereas the cytotoxicity of the gold(I) series is independent of these factors.

\section{INTRODUCTION}

The clinical success of cisplatin resulted in considerable efforts being directed toward the development of other platinumbased therapeutics. ${ }^{1}$ However, the need to overcome the adverse side effects and intrinsic or acquired resistance to these compounds led to the investigation of alternative metals for their therapeutic potential. ${ }^{2}$ Ruthenium(III) complexes imidazolium [trans-tetrachloro( $1 \mathrm{H}$-imidazole $)(\mathrm{S}$-dimethyl sulfoxide)ruthenate(III) $]^{3}$ and indazolium trans-[tetrachlorobis $(1 \mathrm{H}$ indazole)ruthanate(III)] (KP1019) ${ }^{4-6}$ and its sodium analogue $(\mathrm{NKP} 1339)^{7}$ have completed phase I and I/II clinical trials. Ruthenium(II) organometallic compounds have also attracted attention because they exhibit a number of promising pharmacological properties. ${ }^{8-10}$ For example, the so-called RAPTA complexes (Figure 1), ${ }^{11}$ of the general formula $\left[\mathrm{Ru}\left(\eta^{6}\right.\right.$ arene $\left.)(\mathrm{PTA}) \mathrm{X}_{2}\right]$ (PTA = 1,3,5-triaza-7-phosphaadamantane), and the $\mathrm{RAED}$ complexes, $\left[\mathrm{Ru}\left(\eta^{6} \text {-arene }\right)(\mathrm{en}) \mathrm{Cl}\right]^{+}(\mathrm{en}=$ ethylenediamine), ${ }^{12}$ have been particularly well studied for their anticancer properties. RAPTA-C ${ }^{13}$ and RAED-C, ${ }^{14}$ (where $\mathrm{C}=p$-cymene) along with their derivatives exhibit an array of promising in vitro and in vivo properties. ${ }^{9,12,13,15-19}$ Interestingly, crystallographic studies on the nucleosome core particle have shown that the choice of ligand strongly influences the biomolecular target of ruthenium(II) arene complexes with RAED-C preferentially binding to DNA and RAPTA-C binding to the histone proteins. ${ }^{20,21}$ Mononuclear gold(I) phosphine complexes have been evaluated for anticancer properties and exhibit promising activity. ${ }^{22-25}$ Auranofin (1-thio- $\beta$-D-glucopyranose-2,3,4,6-tetraacetato- $S$ )(triethylphosphine)gold(I) (Figure 1 ), which is used clinically for the treatment of rheumatoid arthritis, $^{25,26}$ is currently being repositioned as an anticancer drug. $^{27-32}$ Similar to RAPTA complexes, ${ }^{33,34}$ auranofin preferentially binds to cysteine-rich proteins such as thioredoxin reductase $(\operatorname{TrX}) .^{35-37}$

Multinuclearity, i.e., covalently connecting two or more metal centers via an appropriate linker, emerged as an approach to introducing new modes of action to overcome resistance in chemoresistant cancers. ${ }^{38}$ The trinuclear platinum compound $\left[\left\{\text { trans }-\mathrm{PtCl}\left(\mathrm{NH}_{3}\right)_{2}\right\}_{2}-\mu-\left(\right.\right.$ trans $-\mathrm{Pt}\left(\mathrm{NH}_{3}\right)_{2}\left\{\mathrm{H}_{2} \mathrm{~N}\left(\mathrm{CH}_{2}\right)_{6}\right.$ $\left.\left.\mathrm{NH}_{2}\right\}_{2}\right]^{4+}$ (BBR3464; Figure 1) can overcome cisplatin resistance, and it exhibits a profile of antitumor efficacy distinct from that of cisplatin in a number of preclinical models. ${ }^{39}$ However, despite successfully passing phase I clinical trials, BBR3464 failed a phase II evaluation, with only a minor response observed in small lung cancer and gastric/gastroesophageal adenocarcinoma. ${ }^{40,41}$

A growing number of multinuclear ruthenium(II) and gold(I) complexes have also been reported. ${ }^{38}$ Interest in homobimetallic ruthenium(II) complexes has focused on the structure-reactivity investigations and the use of bioactive bridging ligands such as thiosemicarbazones. ${ }^{42,43}$ The influence of the spacer length on in vitro anticancer activity has previously been explored using bis(pyridinone)alkane linkers $\left(\eta^{6}\right.$-p-cymene $) \mathrm{Ru}\left(\mathrm{O}, \mathrm{O}-\mathrm{C}_{6} \mathrm{H}_{5} \mathrm{O}_{2} \mathrm{~N}\left(\mathrm{CH}_{2}\right)_{n} \mathrm{NC}_{6} \mathrm{H}_{5} \mathrm{O}_{2}-\mathrm{O}, \mathrm{O}\right) \mathrm{Ru}$ ( $\eta^{6}$-p-cymene) (RU1, with $n=3,6,12$; Figure 1$)$, where the cytotoxicity correlates to the lipophilicity, which increases with increasing linker length. ${ }^{44}$ Both proteins and DNA were identified as possible targets for the dinuclear ruthenium(II) complexes, which hydrolyze rapidly to form active diaqua

Received: May 3, 2017

Published: July 31, 2017 


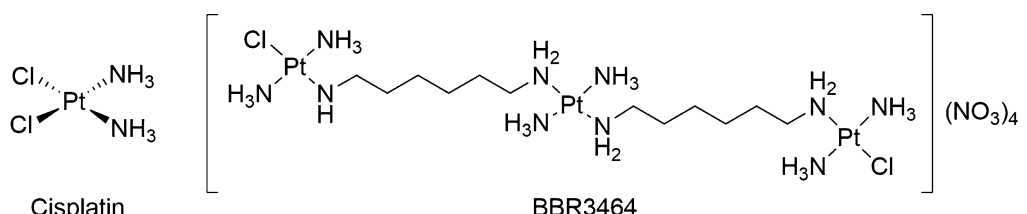

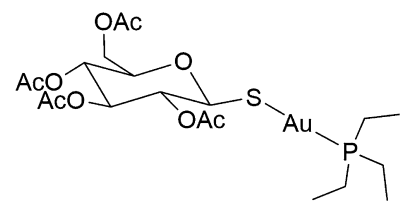

Auranofin

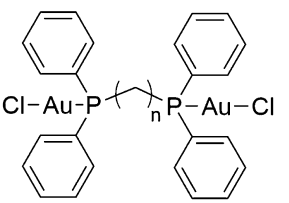

$\mathrm{AU} 1$

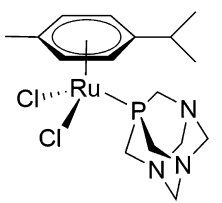

RAPTA-C

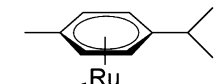<smiles>CO[Ga]O[AlH2]</smiles><smiles>Cc1[nH]ccc(=O)c1OCCl</smiles><smiles>[AlH2]NC1CCCC1</smiles><smiles></smiles>

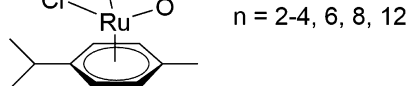

Figure 1. Selected examples of dinuclear complexes inspired by well-known monometallic drugs.

Scheme 1. Synthesis of the Diphosphine Ligands (1a-7a), Diruthenium(II) p-Cymene Complexes (1b-7b), Digold(I) Chloride Intermediates $(1 \mathrm{c}-7 \mathrm{c})$, and Digold(I) $\beta$-D-Thioglucosetetraacetate Complexes $(1 \mathrm{~d}-7 \mathrm{~d})$, Where $n=1-6$ and 8

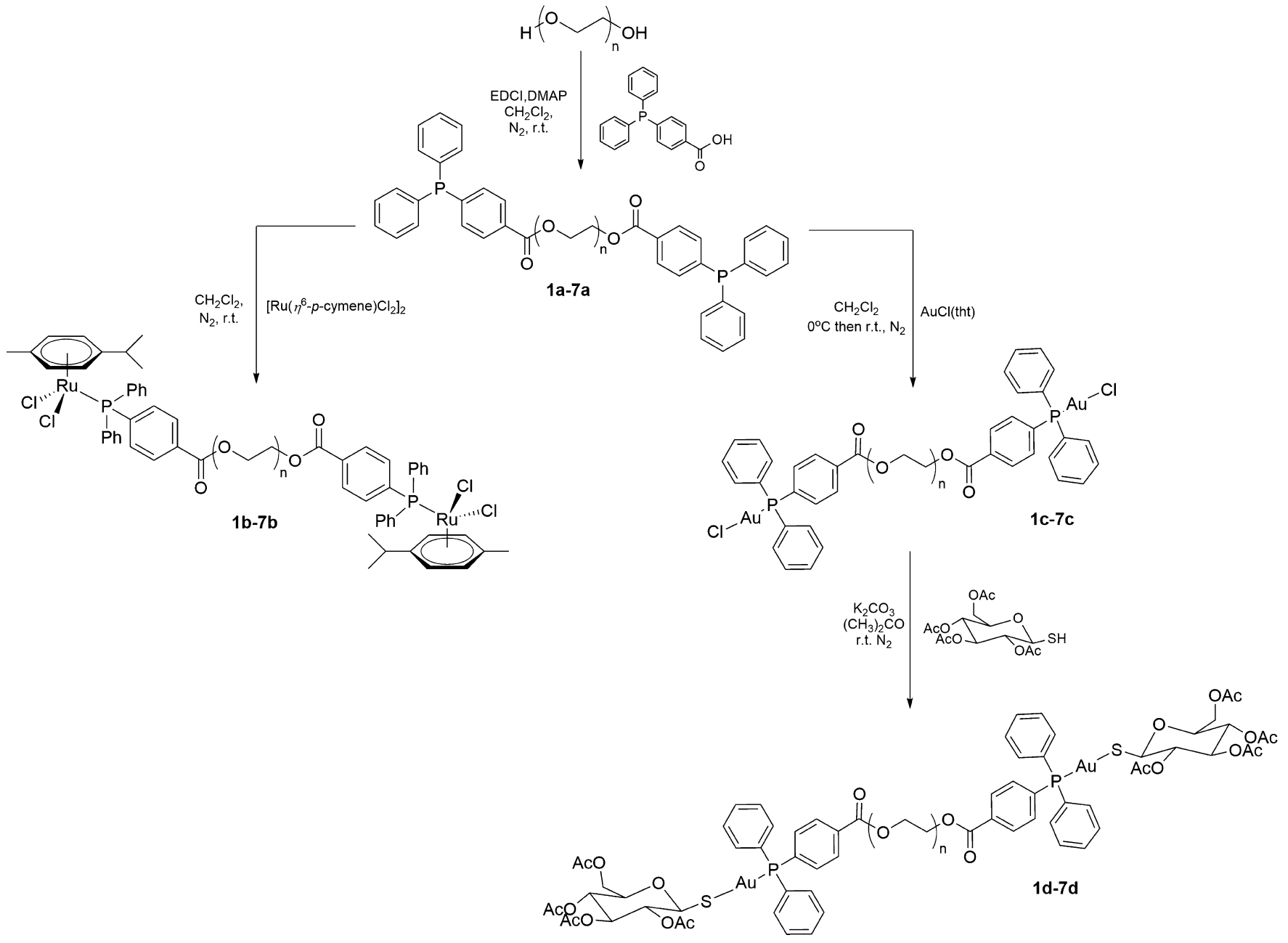

species. ${ }^{45}$ Interestingly, the cytotoxicity of the complex with the longest bridging ligand was attributed to the ability of the complex to form both DNA-DNA and DNA-protein crosslinks. ${ }^{46}$ A RAED-type binuclear complex, [( $\mathrm{Ru}\left(\eta^{6}\right.$-biphenyl)$\left.\mathrm{Cl}(\mathrm{en}))_{2}\left(\mathrm{CH}_{2}\right)_{6}\right]^{2+}$, similarly bearing an alkyl linker, has been shown to form interstrand DNA cross-links. ${ }^{47}$ Flexible alkyl spacers used in acylpyrazolonato-bridged ruthenium(II) com- plexes led to complexes with a higher cytotoxicity than those of related compounds with rigid phenyl spacers. ${ }^{48}$ Attempts were made to investigate poly(ethylene glycol) (PEG) linkers; however, the bis(nicotinate)/bis(isonicotinate) ligands were unstable in solution. ${ }^{49}$ Homobinuclear ruthenium(II) complexes linked by different stereochemically configured 1,2diphenylethylenediamine spacers exhibit open and closed 
Scheme 2. Synthesis of Mononuclear Ruthenium(II) p-Cymene ( 8 b and 9b) and Gold(I) (8d and 9d) Complexes

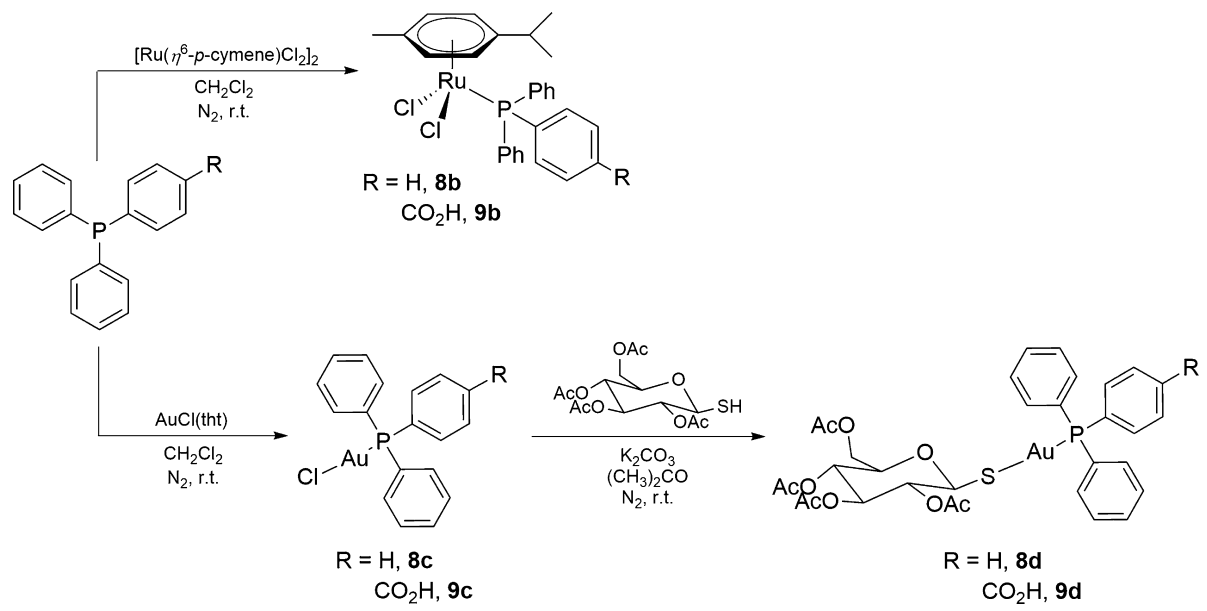

conformations. The dinuclear complexes are considerably more cytotoxic than the monomers but did not display cancer cell selectivity. ${ }^{50}$ Consequently, a strategy was subsequently developed to generate dinuclear ruthenium(II) complexes directly in cancer cells. ${ }^{51}$

Significant efforts have also been devoted to the development of diphosphinegold(I) complexes. ${ }^{52-58}$ However, little attention has been paid to the nature of the linker in homobinuclear gold(I) complexes. A series of $\left[(\mathrm{AuCl})_{2}\left(\mathrm{Ph}_{2} \mathrm{P}-\left(\mathrm{CH}_{2}\right)_{n} \mathrm{PPh}_{2}\right)\right]$ (where $n=1-6)$ complexes were prepared and evaluated in vitro against murine B16 melanoma cells (AU1; Figure 1). The cytotoxicity initially decreases with the linker length, $n=1(6$ $\mu \mathrm{M})$ and $2(8 \mu \mathrm{M})$, up to $n=3$, where a plateau is reached $(n=$ $\left.3-6, \mathrm{IC}_{50}=2-3 \mu \mathrm{M}\right) .{ }^{59}$ Alkyl linkers were also assessed in phosphine-bridged dinuclear gold(I) alkynyl complexes linked via $\left(\mathrm{CH}_{2}\right)_{n}$ (where $n=1$ and 4 ) groups. ${ }^{60}$ More recently, investigations into the lipophilicity have included the exchange of hydrophobic $\mathrm{PPh}_{3}$ ligands with more lipophilic $\mathrm{PEt}_{3}$ ligands in dinuclear phosphinegold(I) sulfanylcarboxylate complexes resulting in lower $\mathrm{IC}_{50}$ values against selected cell lines. ${ }^{61}$ Other investigations include the optimization of $\operatorname{TrX}$ inhibition bridging bis(N-heterocyclic carbene) ligands, ${ }^{62-64}$ alkylnyl ligands, ${ }^{60,65-67}$ and thiocarbonates. ${ }^{68,69}$

Because the use of alkyl chains produced a general dependence between the linker length and cytotoxicity, correlating with increasing lipophilicity, we decided to evaluate the effect of PEG chains. Herein, we report the synthesis, structural characterization, and antiproliferative activity of two series of dinuclear complexes based on ruthenium(II) and gold(I) systems. The diruthenium(II) and digold(I) centers are linked via PEG chains of varying length, while the basic structures of the parent drugs RAPTA-C and auranofin are maintained. This strategy allows the influence of the linker length on the cytotoxicity to be studied and compared to their monometallic precursors.

\section{RESULTS AND DISCUSSION}

Two series of homobinuclear ruthenium(II) $p$-cymene, $\mathbf{1} \mathbf{b}-\mathbf{7 b}$, and gold(I), 1d-7d, derivatives were prepared using the routes shown in Scheme 1. Universal diphosphine ligands 1a-7a were synthesized via an esterification reaction between 4(diphenylphosphanyl)benzoic acid and the appropriate ethylene glycol in the presence of $\mathrm{N}$-ethyl- $\mathrm{N}^{\prime}$-[3-(dimethylamino)propyl]carbodiimide hydrochloride (EDCI), a coupling agent, and 4-(dimethylamino)pyridine (DMAP), a basic catalyst. Ligands $1 \mathrm{a}-7 \mathbf{a}$ were isolated by chromatographic purification in moderate yields $(36-64 \%)$.

The diruthenium(II) $p$-cymene complexes $\mathbf{1} \mathbf{b}-\mathbf{7 b}$ were obtained in high yield (96-98\%) in a single step from the reaction of the appropriate ligands $1 \mathrm{a}-7 \mathbf{a}$ with the $\left[\mathrm{Ru}\left(\eta^{6}-p\right.\right.$ cymene $\left.\mathrm{Cl}_{2}\right]_{2}$ dimer, under inert conditions in dry dichloromethane $\left(\mathrm{CH}_{2} \mathrm{Cl}_{2} ;\right.$ Scheme 1$)$. The binuclear gold(I) $\beta$-Dthioglucosetetraacetate complexes $\mathbf{1 d}-\mathbf{7 d}$ were prepared by following a two-step strategy (Scheme 1). The intermediate binuclear gold(I) chloride complexes, $1 \mathrm{c}-7 \mathrm{c}$, were obtained in good yield (95-98\%) from direct reaction of the appropriate diphosphine ligands $\mathbf{1 a}-7 \mathbf{a}$ with $\mathrm{Au}^{\mathrm{I}} \mathrm{Cl}$ (tht) (tht = tetrahydrothiophene), freshly prepared following an adapted literature procedure. ${ }^{70,71}$ The subsequent reaction of $1 \mathbf{c}-7 \mathbf{c}$ with the $\beta$-Dthioglucosetetraacetate ligand under basic conditions $\left(\mathrm{K}_{2} \mathrm{CO}_{3}\right)$ in acetone or ethanol $(\mathrm{EtOH}) /$ water $\left(\mathrm{H}_{2} \mathrm{O}\right)$ affords the desired gold(I) complexes $\mathbf{1 d - 7 d}$ in good yield (84-96\%). ${ }^{71,72}$

Mononuclear complexes were also prepared to provide a structure-activity comparison between the binuclear complexes and the parent drugs, RAPTA-C and auranofin. The mononuclear ruthenium(II) $p$-cymene ( $\mathbf{8 b}$ and $\mathbf{9 b}$ ) and gold(I) (8d and 9d) complexes, containing 4-(diphenylphosphanyl)benzoic acid and triphenylphosphine ligands, were prepared from the direct reaction of phosphines with the $\left[\mathrm{Ru}\left(\eta^{6}-p\right.\right.$ cymene $\left.\mathrm{Cl}_{2}\right]_{2}$ dimer and $\mathrm{Au}{ }^{\mathrm{I}} \mathrm{Cl}(\mathrm{tht})$ precursors, respectively (Scheme 2).

All compounds were characterized by ${ }^{1} \mathrm{H},{ }^{31} \mathrm{P}$, and ${ }^{13} \mathrm{C}$ NMR spectroscopy, mass spectrometry (MS), and elemental analysis. In the ${ }^{31} \mathrm{P}$ NMR spectra, the phosphine ligands $1 \mathrm{a}-7$ a produce a singlet between -4.99 and $-5.08 \mathrm{ppm}$. In the binuclear complexes, the peaks shift to higher frequencies, with singlet resonances observed at $24.94-25.00 \mathrm{ppm}$ for $\mathbf{1 b}-7 \mathbf{b}, 33.01-$ $33.14 \mathrm{ppm}$ for $1 \mathrm{c}-7 \mathrm{c}$, and $38.71-38.79 \mathrm{ppm}$ for $1 \mathrm{~d}-7 \mathrm{~d}$, confirming coordination of the phosphorus centers to the metal ions. The mononuclear complexes also give rise to singlets observed in the same range as the binuclear species, i.e., at $24.18 \mathrm{ppm}$ for $\mathbf{8 b}, 25.28 \mathrm{ppm}$ for $\mathbf{9 b}, 33.19 \mathrm{ppm}$ for $\mathbf{8 c}, 33.21$ ppm for 9c, $38.83 \mathrm{ppm}$ for $\mathbf{8 d}$, and $38.82 \mathrm{ppm}$ for $\mathbf{9 d}$.

The ${ }^{1} \mathrm{H}$ NMR spectra of the ligands $1 \mathrm{a}-7 \mathbf{a}$ and complexes also show distinct differences. The multiplet corresponding to eight protons ortho to the $\mathrm{C}-\mathrm{P}$ bond on the phenyl rings shifts from 7.29-7.39 to 7.76-7.83 ppm $\left(\Delta \delta_{\mathrm{H}} \approx 0.4 \mathrm{ppm}\right)$, with a larger shift to $7.85-7.98 \mathrm{ppm}\left(\Delta \delta_{\mathrm{H}} \approx 0.6 \mathrm{ppm}\right)$ observed for 
the four ortho protons on the functionalized ring. Similarly, a shift of $\Delta \delta_{\mathrm{H}} \approx 0.2 \mathrm{ppm}$ to higher frequencies is observed, from $7.29-7.39 \mathrm{ppm}(1 \mathrm{a}-7 \mathrm{a})$ to $7.45-7.60 \mathrm{ppm}(\mathbf{1}-7 \mathrm{c}$ and $1 \mathrm{~d}-$ $7 \mathrm{~d}$ ), corresponding to the 12 protons ortho to the $\mathrm{C}-\mathrm{P}$ bond in the gold(I) complexes. Complex $8 \mathbf{b}$ possesses two multiplets in the aromatic region: $7.77-7.85 \mathrm{ppm}$, corresponding to the six phenyl protons ortho to the C-P bond, and 7.46-7.32 ppm, corresponding to the nine meta and para protons. In contrast, the ${ }^{1} \mathrm{H}$ NMR spectra of complexes $\mathbf{8 c}$ and $\mathbf{8 d}$ contain only one aromatic multiplet that corresponds to all 15 protons of the triphenylphosphine ligand (8c, 7.42-7.58 ppm; 8d, $7.42-7.59 \mathrm{ppm}$ ). Complex $9 \mathrm{~b}$ possesses a multiplet at $7.97-$ $8.00 \mathrm{ppm}$ corresponding to the four protons of the functionalized ring, whereas in $9 \mathrm{c}$ and $\mathbf{9 d}$, the multiplets corresponding to the two protons ortho to the $\mathrm{C}-\mathrm{P}$ bond are observed at 8.05-8.19 ppm in 9c and 7.81-7.90 ppm in 9d.

Coordination of the ligands to the metal ions is denoted by the peaks corresponding to the carbon atoms directly connected to the phosphorus center shifting to lower frequencies (with increased ${ }^{1} J_{\mathrm{C}, \mathrm{P}}$ coupling constants) in the ${ }^{13} \mathrm{C}$ NMR spectra. In the diruthenium(II) $p$-cymene complexes $\mathbf{1 b}-7 \mathbf{b}$, a shift of $\Delta \delta_{\mathrm{C}} \approx 4.9 \mathrm{ppm}$ and coupling of $\Delta^{1} J_{\mathrm{C}, \mathrm{P}} \approx 28$ $\mathrm{Hz}$ were observed for the two $\mathrm{C}-\mathrm{P}$ carbon atoms on the functionalized ring and $\Delta \delta_{\mathrm{C}} \approx 3 \mathrm{ppm}$ and $\Delta^{1} J_{\mathrm{C}, \mathrm{P}} \approx 34 \mathrm{~Hz}$ for the peaks corresponding to the four $\mathrm{C}-\mathrm{P}$ carbon atoms on the phenyl rings. An analogous effect was observed for the digold(I) complexes, for $1 \mathrm{c}-7 \mathrm{c}$, there is a shift of $\Delta \delta_{\mathrm{C}} \approx 9.6$ ppm and a coupling of $\Delta^{1} J_{\mathrm{C}, \mathrm{P}} \approx 45 \mathrm{~Hz}$, corresponding to the two $\mathrm{C}-\mathrm{P}$ carbon atoms on the functionalized ring and a shift of $\Delta \delta_{\mathrm{C}} \approx 8.5 \mathrm{ppm}$ and $\Delta^{1} J_{\mathrm{C}, \mathrm{P}} \approx 52 \mathrm{~Hz}$ representing the four C-P carbon atoms on the phenyl rings. A comparable, but slightly less pronounced, effect is observed in the ${ }^{13} \mathrm{C}$ NMR spectra of $\mathbf{1 d}-7 \mathbf{d}$, and in the mononuclear complexes, similar changes are also observed.

The impact of increasing PEG chain length on the electronic environment of the metal centers is negligible, with all coupling constants and ${ }^{31} \mathrm{P}$ peaks remaining consistently similar throughout the series.

The most abundant peaks observed in the electrospray ionization MS (ESI-MS) spectra may be assigned to $[\mathrm{M}+\mathrm{H}]^{+}$ and $[\mathrm{M}+\mathrm{Na}]^{+}$ions for ligands $\mathbf{1 a}-\mathbf{9 a},[\mathrm{M}-\mathrm{Cl}]^{+}$ions for $\mathbf{1 b}-$ $9 b,[\mathrm{M}+\mathrm{Na}]^{+}$ions for $1 \mathrm{c}-8 \mathrm{c},[\mathrm{M}-\mathrm{H}]^{-}$ions for $9 \mathrm{c},[\mathrm{M}-$ $\mathrm{Cl}]^{+}$ions for $\mathbf{1 d - 7 d}$, and $[\mathrm{M}+\mathrm{H}]^{+}$ions for $\mathbf{8 d - 9 d}$.

The solid-state structures of $\mathbf{9 b}$ and $\mathbf{9 c}$ were established by single-crystal X-ray diffraction analysis, confirming the expected molecular structures. Single crystals of $\mathbf{9 b}$ were grown via the slow evaporation of chloroform from a concentrated solution (Figure 2). 9b contains four independent molecules in each asymmetric unit (Figure S1) compared to the two found for RAPTA-C. ${ }^{73}$ Key bond parameters are compared with those of RAPTA-C (Table 1) and, overall, the arrangement around the ruthenium(II) center is remarkably similar to that of $\mathbf{9 b}$, adopting the familiar half-sandwich three-legged piano-stool geometry. The mean $\mathrm{Ru}-\eta^{6}$ distance is longer in $9 \mathrm{~b}$ (1.706$1.723 \AA)$ than in RAPTA-C (1.692-1.701 $\AA)$, and the same trend is observed for the $\mathrm{Ru}-\mathrm{P}$ bond lengths [9b, 2.363(2)-

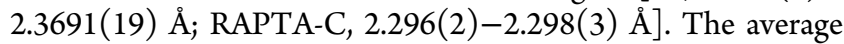
$\mathrm{Ru}-\mathrm{Cl}$ bond lengths and $\mathrm{Cl}-\mathrm{Ru}-\mathrm{Cl}$ angles are similar for both complexes; however, a difference is observed in the average $\mathrm{P}-$ $\mathrm{Ru}-\mathrm{Cl}$ angles, with those in $9 \mathrm{~b}\left(88.78-89.91^{\circ}\right)$ being consistently larger than those in RAPTA-C (84.04-87.25 ). In the crystal of $\mathbf{9 b}$, intermolecular hydrogen bonds between

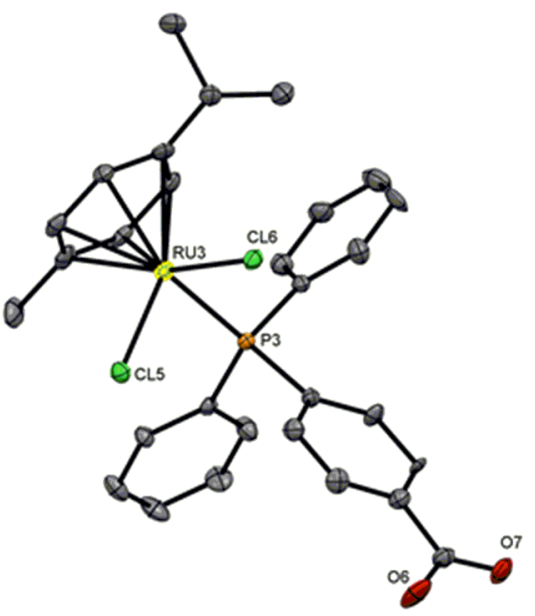

Figure 2. Solid-state structure of one of the four independent molecules in $\mathbf{9 b}$. Thermal ellipsoids are $50 \%$ equiprobability envelopes. Hydrogen atoms and solvent molecules $\left(\mathrm{CHCl}_{3}\right)$ are omitted for clarity.

Table 1. Comparison of Selected Bond Lengths $(\AA)$ and Angles (deg) in RAPTA-C ${ }^{73}$ and $9 b$

\begin{tabular}{lll}
\multicolumn{3}{c}{ RAPTA-C $^{a}$} \\
$\mathrm{Ru}-\eta^{6}$ & $1.692,1.701$ & \multicolumn{1}{c}{$9 \mathbf{b}^{b}$} \\
$\mathrm{Ru}-\mathrm{P}$ & $2.296(2)$, & $2.709,1.723,1.706,1.714$ \\
& $2.298(3)$ & $2.3651(19)$ \\
$\mathrm{Ru}-\mathrm{Cl}_{\text {ave }}$ & $2.421,2.426$ & $2.420,2.420,2.425,2.420$ \\
$\mathrm{Cl}-\mathrm{Ru}-\mathrm{Cl}$ & $87.25(8)$, & $89.85(7), 88.52(7), 89.50(7), 87.51(6)$ \\
& $88.97(9)$ & \\
$\mathrm{P}-\mathrm{Ru}-\mathrm{Cl}_{\text {ave }}$ & $85.26,84.04$ & $89.16,88.78,89.91,89.35$
\end{tabular}

${ }^{a}$ In the crystal of RAPTA-C, there are two independent molecules in the asymmetric unit. ${ }^{b}$ In $9 \mathbf{b}$, there are four independent complexes in the asymmetric unit.

the carboxylic acid groups are observed, leading to the formation of homodimeric assemblies (Figure S2).

The slow diffusion of tetrahydrofuran (THF) into a saturated solution of $9 \mathrm{c}$ in $\mathrm{CDCl}_{3}$ afforded crystals suitable for $\mathrm{X}$-ray diffraction analysis (Figure 3). The bond parameters around the gold(I) center in 9c are presented in Table 2 and compared with those of auranofin. ${ }^{14}$ The $\mathrm{Au}-\mathrm{P}$ bond distance in 9c $[2.233(9) \AA]$ is comparable to the value observed in auranofin

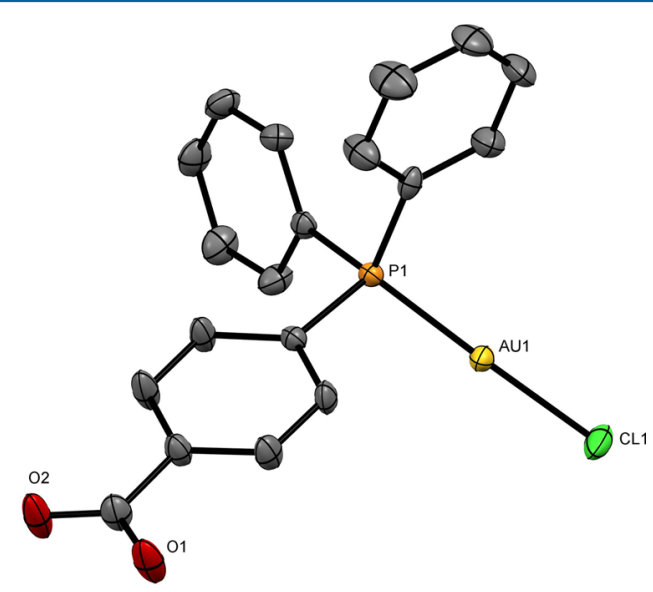

Figure 3. Solid-state structure of 9c. Thermal ellipsoids are 50\% equiprobability envelopes. Hydrogen atoms are omitted for clarity. 
Table 2. Comparison of Selected Bond Lengths (Å) and Angles (deg) in Auranofin ${ }^{74}$ and 9c

\begin{tabular}{llll}
\multicolumn{2}{r}{ auranofin } & \multicolumn{2}{c}{$9 c$} \\
$\mathrm{Au}-\mathrm{P}$ & 2.259 & $\mathrm{Au}-\mathrm{P}$ & $2.233(9)$ \\
$\mathrm{Au}-\mathrm{S}$ & 2.293 & $\mathrm{Au}-\mathrm{Cl}$ & $2.286(10)$ \\
$\mathrm{P}-\mathrm{Au}-\mathrm{S}$ & 173.6 & $\mathrm{P}-\mathrm{Au}-\mathrm{Cl}$ & $178.07(3)$ \\
\hline
\end{tabular}

(2.259 ̊)). Despite the different labile ligands, i.e., thiol versus chloride, the $\mathrm{Au}-\mathrm{Cl}$ bond in 9c [2.286(10) $\AA]$ is similar in length to the $\mathrm{Au}-\mathrm{S}$ bond in auranofin $(2.293 \AA)$. However, the nature of the labile ligand influences the angle around the gold(I) center; the $\mathrm{P}-\mathrm{Au}-\mathrm{Cl}\left[178.07(3)^{\circ}\right]$ angle in $9 \mathrm{c}$ is larger than the auranofin $\mathrm{P}-\mathrm{Au}-\mathrm{S}$ angle $\left(173.6^{\circ}\right)$. The crystal network in 9c reveals dimeric arrangements due to intermolecular hydrogen-bonding interactions of the carboxylic acid group (Figure S3).

The lipophilicity has previously been correlated to increasing cytotoxicity in dinuclear ruthenium(II) complexes (RU1; Figure 1). ${ }^{45}$ Consequently, the partition coefficients $(\log P)$ of PEG chains and alkyl chains were calculated. ${ }^{75,76}$ As expected, the lipophilicity of the alkanes increases with increasing chain length (Chart 1), a trend that is transferred

Chart 1. Calculated Partition Coefficients for Alkyl Chains and PEG Chains as a Function of the Length ${ }^{a}$

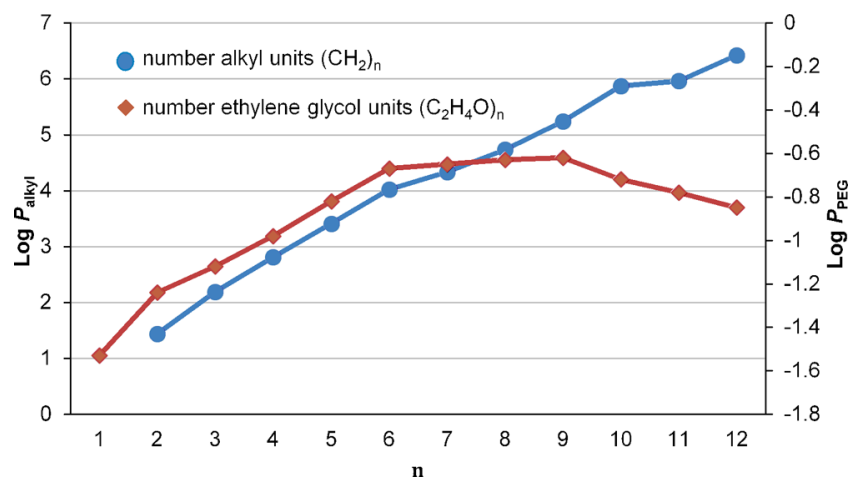

${ }^{a}$ The calculated $\log P_{\text {alkyl }}$ value of methane $(n=1)$ was omitted for clarity.

to diruthenium complexes bearing alkyl linkers of the structure $\left(\eta^{6}\right.$-p-cymene $) \mathrm{Ru}\left(\mathrm{O}, \mathrm{O}-\mathrm{C}_{6} \mathrm{H}_{5} \mathrm{O}_{2} \mathrm{~N}\left(\mathrm{CH}_{2}\right)_{\mathrm{n}} \mathrm{NC}_{6} \mathrm{H}_{5} \mathrm{O}_{2}-\mathrm{O}, \mathrm{O}\right) \mathrm{Ru}$ ( $\eta^{6}$-p-cymene) $(n=3,6$, and 12$) .{ }^{45}$ In contrast, PEG chains have limited lipophilicity, with the hydrophobicity increasing with the chain length up to hexakis(ethylene glycol), where a plateau is reached. According to calculations, octakis(ethylene glycol) is the most lipophilic with a $\log P$ value of -0.63 , and longer PEG chains become increasingly hydrophilic. The plateau, consisting of PEG chains 6-9, have similar log $P$ values in the range -0.62 to -0.67 . $\log P_{\text {octanol } / \mathrm{H}_{2} \mathrm{O}}$ values were determined experimentally for $\mathbf{1 b}-\mathbf{7 b}$ and $\mathbf{1 d}-\mathbf{7 d}$ using the shake-flask method (Table 1). ${ }^{77}$ The $\log P$ values reside in the lipophilic range for both series. Digold(I) $\beta$-D-thioglucosetetraacetate complexes $\mathbf{1 d}-\mathbf{7 d}$ are more hydrophilic than their ruthenium counterparts $\mathbf{1 b}-\mathbf{7 b}$ because of the presence of two $\beta$-D-thioglucosetetraacetate ligands. For the shorter chain lengths, $\mathbf{1} \mathbf{b}-\mathbf{4 b}$ and $\mathbf{1 d}-\mathbf{3 d}$, the lipophilicity was shown to increase with increasing chain length for both series. However, for complexes bearing longer chain lengths, $\mathbf{5 b}-\mathbf{7 b}$ and $\mathbf{5 d}-\mathbf{7 b}$, the $\log P$ values remain essentially constant despite increasing chain length, values of which are $1.4-1.5$ and $0.3-0.4$, respectively.

The cytotoxicity of the $1 \mathbf{a}-7 \mathbf{a}$ ligands and ruthenium(II) $p$ cymene $\mathbf{1 b}-\mathbf{9 b}$ and gold(I) $\beta$-D-thioglucosetetraacetate $\mathbf{1 d - 9 d}$ complexes was assessed against human ovarian carcinoma cell lines, A2780 and A2780cisR, with the latter having acquired resistance to cisplatin and nontumoral human embryonic kidney (HEK-293) cells using the 3-(4,5-dimethyl-2-thiazolyl)2,5-diphenyl-2H-tetrazolium bromide (MTT) assay (Table 3 ). The cytotoxicities of 4-(diphenylphosphanyl)benzoic acid, triphenylphosphine, auranofin, cisplatin, and RAPTA-C were evaluated for comparison purposes. Elemental analysis indicates that $\mathrm{CH}_{2} \mathrm{Cl}_{2}$ and $\mathrm{CDCl}_{3}$ are present in some compounds; therefore, their cytotoxicities were evaluated at $\mathrm{IC}_{50}$ concentration, and they were found to be inactive.

All compounds were predissolved in dimethyl sulfoxide (DMSO), and their stability in this solvent was confirmed via ${ }^{1} \mathrm{H}$ and ${ }^{31} \mathrm{P}$ NMR spectroscopy (Figures S4-S7), before being immediately diluted into the appropriate cell culture medium. Further stability studies under pseudocell culture conditions of $100 \mathrm{mM} \mathrm{NaCl}$ in $\mathrm{H}_{2} \mathrm{O}$ and 5\% DMSO were conducted on $2 \mathrm{~d}$, 4d, and $6 \mathrm{~d}$ complexes over $72 \mathrm{~h}$. The stability was monitored via ESI-MS(+) (Figures S8-S10), and all complexes showed good stability under these conditions.

The original RAPTA series possess low cytotoxicities with IC $_{50}$ values of $>200 \mu \mathrm{M}$ against a range of cell lines. ${ }^{11}$ As discussed above, the structures of $\mathbf{9 b}$ and RAPTA-C are comparable, and the similarities are reflected in their cytotoxicities, with $\mathrm{IC}_{50}$ values $>200 \mu \mathrm{M}$ determined against all tested cell lines. However, the mononuclear ruthenium(II) complex $\mathbf{8 b}$, bearing a hydrophobic triphenylphosphine ligand, is considerably more cytotoxic than $9 \mathbf{b}$, with an $\mathrm{IC}_{50}$ of $42 \pm 1$ $\mu \mathrm{M}$. A similar trend is present with $\mathbf{8 d}$ and $\mathbf{9 d}$, with $\mathbf{9 d}$ being ca. 14-fold more cytotoxic than $\mathbf{8 d}$. The free ligands triphenylphosphine and 4-(diphenylphosphanyl)benzoic acid also present respective $\mathrm{IC}_{50}$ values of $85 \pm 7$ and $>200 \mu \mathrm{M}$ against the A2780 cell line; the differences may be attributed to the differences in the lipophilicity. However, the impact of global charge may be of influence.

The diphosphine ligands $1 \mathbf{a}-7 \mathbf{a}$ are inactive at concentrations of up to $500 \mu \mathrm{M}$ on the three cell lines. The dinuclear complexes $\mathbf{1 b}-7 \mathbf{b}$ and $\mathbf{1 d}-\mathbf{7 d}$ are considerably more cytotoxic than the mononuclear complexes $\mathbf{9 b}$ and $\mathbf{9 d}$ against all cell lines. The complexes containing the mono(ethylene glycol) linker (1b and $\mathbf{1 d}$ ) are considerably less cytotoxic than the complexes with longer linkers $(\mathbf{2} \mathbf{b}-\mathbf{7 b}$ and $\mathbf{2} \mathbf{d}-\mathbf{7 d})$. In contrast, in a series of diruthenium(II) $p$-cymene complexes with bridging bis(nicotinate)/bis(isonicotinate) ligands, only the complex with the shortest mono(ethylene glycol) linker possessed moderate activity against a range of cell lines including the human melanoma (518A2) cell line $\left(\mathrm{IC}_{50}=53 \pm\right.$ $1 \mu \mathrm{M}){ }^{49}$

The diruthenium(II) $p$-cymene complexes $\mathbf{2 b}-\mathbf{7 b}$ exhibit $\mathrm{IC}_{50}$ values in the low micromolar range against the $\mathrm{A} 2780$ cell line. The cytotoxicity toward the cisplatin-resistant cell line (A2780cisR) remains in the low micromolar range, with up to 2 -fold loss in cytotoxicity compared to the A2780 cisplatinsensitive cell line for $\mathbf{3} \mathbf{b}$ and $\mathbf{5 b}$. No selectivity was observed toward the cancer cell lines, with the values obtained for the nontumorigenic HEK-293 cell line being similar in magnitude. The cytotoxicities of these dinuclear complexes are comparable to those of previously investigated series including the series of rigid RAPTA-type dinuclear complexes, linked via the 
Table 3. Calculated $\log P$ Values and in Vitro Antiproliferative Activities of Compounds $1 \mathrm{~b}-9 \mathrm{~b}$ and $1 \mathrm{~d}-9 \mathrm{~d}$ against Human Ovarian Carcinoma (A2780), Human Ovarian Carcinoma Cisplatin Resistant (A2780cisR), and Human Embryonic Kidney 293 (HEK-293) Cell Lines after $72 \mathrm{~h}$ of Exposure ${ }^{a}$

\begin{tabular}{|c|c|c|c|c|}
\hline compound & $\log P_{\text {octanol } / \mathrm{H}_{2} \mathrm{O}}$ & A2780 & A2780cisR & HEK-293 \\
\hline $1 b$ & $1.3 \pm 0.1$ & $60 \pm 2$ & $110 \pm 3$ & $66 \pm 1$ \\
\hline $2 b$ & $1.3 \pm 0.1$ & $10 \pm 0.1$ & $11.0 \pm 0.7$ & $12.2 \pm 0.5$ \\
\hline $3 b$ & $1.6 \pm 0.1$ & $19.4 \pm 0.3$ & $37.6 \pm 1.6$ & $19.6 \pm 1.1$ \\
\hline $4 b$ & $1.8 \pm 0.3$ & $11.3 \pm 0.1$ & $14.6 \pm 0.3$ & $14.7 \pm 0.9$ \\
\hline $5 \mathbf{b}$ & $1.4 \pm 0.05$ & $6.4 \pm 0.7$ & $12.9 \pm 3.7$ & $6.8 \pm 0.2$ \\
\hline $6 b$ & $1.4 \pm 0.02$ & $7.3 \pm 0.3$ & $10.5 \pm 0.1$ & $9.1 \pm 0.1$ \\
\hline $7 \mathbf{b}$ & $1.5 \pm 0.02$ & $11.6 \pm 0.9$ & $14.1 \pm 7.7$ & $14.2 \pm 0.3$ \\
\hline $8 b$ & & $42 \pm 1$ & $35 \pm 7$ & $47 \pm 1$ \\
\hline $9 b$ & & $>200$ & $>200$ & $>200$ \\
\hline 1d & $0.4 \pm 0.3$ & $1.5 \pm 0.1$ & $4.7 \pm 0.1$ & $3.3 \pm 0.3$ \\
\hline $2 \mathrm{~d}$ & $0.9 \pm 0.1$ & $0.22 \pm 0.03$ & $0.67 \pm 0.02$ & $1.2 \pm 0.1$ \\
\hline $3 d$ & $0.7 \pm 0.2$ & $0.19 \pm 0.02$ & $0.91 \pm 0.01$ & $1.2 \pm 0.1$ \\
\hline $4 d$ & $0.6 \pm 0.1$ & $0.19 \pm 0.02$ & $1.1 \pm 0.1$ & $1.2 \pm 0.1$ \\
\hline $5 d$ & $0.3 \pm 0.2$ & $0.22 \pm 0.02$ & $1.4 \pm 0.1$ & $1.4 \pm 0.1$ \\
\hline $6 d$ & $0.4 \pm 0.3$ & $0.17 \pm 0.01$ & $1.4 \pm 0.04$ & $1.4 \pm 0.1$ \\
\hline $7 \mathrm{~d}$ & $0.3 \pm 0.2$ & $0.25 \pm 0.02$ & $1.4 \pm 0.4$ & $1.4 \pm 0.1$ \\
\hline $8 d$ & & $0.54 \pm 0.07$ & $1 \pm 0.1$ & $1.8 \pm 0.2$ \\
\hline 9d & & $6.9 \pm 0.8$ & $12.0 \pm 2$ & $11.7 \pm 0.4$ \\
\hline$\left(\mathrm{C}_{6} \mathrm{H}_{5}\right)_{2} \mathrm{PC}_{6} \mathrm{H}_{4} \mathrm{CO}_{2} \mathrm{H}$ & & $>200$ & $>200$ & $>200$ \\
\hline $\mathrm{P}\left(\mathrm{C}_{6} \mathrm{H}_{5}\right)_{3}$ & & $85 \pm 7$ & & \\
\hline cisplatin & & $1.3 \pm 0.2$ & $11 \pm 1$ & $9 \pm 1$ \\
\hline RAPTA-C & & $>200$ & $>200$ & $>200$ \\
\hline auranofin & & $1.3 \pm 0.5$ & $1.5 \pm 0.5$ & $1.9 \pm 0.6$ \\
\hline
\end{tabular}

functionalization of the $\eta^{6}$-arene, where the most active complex has an $\mathrm{IC}_{50}$ of $3.7 \pm 0.6 \mu \mathrm{M}$ against the $\mathrm{A} 2780$ cell line. ${ }^{50}$

The relationship between the linker length and cytotoxicity on the A2780 cell line shows increasing cytotoxicity with increasing linker length between $\mathbf{3 b}$ and $\mathbf{5 b}$, which correlates with the increasing lipophilicity of the linkers. However, $\mathbf{2} \mathbf{b}$ with the bis(ethylene glycol) linker is ca. 2-fold more cytotoxic than $\mathbf{3 b}$. The lipophilicity is essentially constant for compounds $\mathbf{6 b}$ and $7 \mathbf{b}$, with the $\mathrm{IC}_{50}$ values being $7.3 \pm 0.3$ and $11.6 \pm 0.9$ $\mu \mathrm{M}$, respectively.

The digold(I) $\beta$-D-thioglucosetetraacetate complexes $\mathbf{1 d - 7 d}$ are highly cytotoxic on all three tested cell lines, with $\mathrm{IC}_{50}$ values in the low micromolar range. Compounds $\mathbf{2 d - 7} \mathbf{d}$ are highly cytotoxic against A2780 cells (with $\mathrm{IC}_{50}$ values between 0.17 and $0.25 \mu \mathrm{M}$ ), while being significantly less active, up to 8fold, on the cisplatin-resistant A2780cisR cell line. The $\mathrm{IC}_{50}$ values of $\mathbf{1 d}-\mathbf{7 d}$ on nontumorigenic HEK-293 cells are very similar to those on A2780cisR cells. Interestingly, no major differences are observed between the activities of the digold(I) complexes $\mathbf{2 d - 7 d}$ and the mononuclear complex $\mathbf{8 d}$, containing the hydrophobic $\mathrm{PPh}_{3}$ ligand, whereas 9d, with the more hydrophilic phosphine ligand, i.e., $\left(\mathrm{C}_{6} \mathrm{H}_{5}\right)_{2} \mathrm{PC}_{6} \mathrm{H}_{4} \mathrm{CO}_{2} \mathrm{H}$, is ca. 35 -fold less cytotoxic in $\mathrm{A} 2780$ cells and ca. 10-fold less cytotoxic in A2780cisR and HEK-293 cells. With the exception of complexes $1 \mathbf{d}$ and $9 \mathbf{d}$, the activities are in the same order as those of auranofin.

Complexes $\mathbf{2 d - 7 d}$ display a narrow range of $\mathrm{IC}_{50}$ values between $0.17 \pm 0.01$ and $0.25 \pm 0.02 \mu \mathrm{M}$ and, thus, there is no discernible correlation with the linker length and associated lipophilicity. However, the series is significantly more cytotoxic than $\left[(\mathrm{AuCl})_{2}\left(\mathrm{Ph}_{2} \mathrm{P}-\left(\mathrm{CH}_{2}\right)_{n} \mathrm{PPh}_{2}\right)\right]$ (where $\left.n=1-6\right)$, in which the most active complexes $(n=3,5$, and 6$)$ have $\mathrm{IC}_{50}$ values of ca. $2 \mu \mathrm{M}$, again murine B16 melanoma cells. ${ }^{59} \mathrm{~A}$ trend was observed in this alkyl-linked series with 3-fold $(n=1)$ and 4fold $(n=2)$ higher cytotoxicities for the more lipophilic complexes. ${ }^{59}$ The series is also significantly more cytotoxic than the phosphine-bridged dinuclear gold(I) alkynyl complexes bearing alkyl linkers, where no correlation was observed between the cytotoxicity and linker length. ${ }^{60}$

\section{CONCLUDING REMARKS}

The synthesis of two series of homobinuclear RAPTA-like ruthenium(II) $p$-cymene complexes $\mathbf{1 b} \mathbf{b} \mathbf{7 b}$ and auranofin-like gold(I) complexes $\mathbf{1 d - 7 d}$, linked via diphosphine-modified PEG chains of varying length, was successfully achieved. The antiproliferative activity of these compounds was determined against tumorigenic and nontumorigenic cell lines, and a distinct increase the cytotoxicity was observed for both series compared to the mononuclear precursors $9 \mathrm{~b}$ and $\mathbf{9 d}$. There is a correlation between the lipophilicity and cytotoxicity of the diruthenium(II) complexes, which reaches a plateau where the lipophilicity no longer increases with the length of the PEG chain, i.e., when $n=6$. In contrast, the cytotoxicities of all of the digold(I) complexes lie within a narrow range and are not readily correlated to the linker length and associated lipophilicity.

\section{EXPERIMENTAL SECTION}

Materials. $\mathrm{RuCl}_{3} \cdot 3 \mathrm{H}_{2} \mathrm{O}$ was purchased from Precious Metals Online. All other chemical reagents were purchased from Aldrich, Alfa Aesar, Acros, and TCI Chemicals and used without further purification. $\left[\mathrm{Ru}\left(\eta^{6}-p \text {-cymene }\right) \mathrm{Cl}_{2}\right]_{2}{ }^{78}$ and $\mathrm{AuCl}(\mathrm{tht})^{70,71}$ were prepared following literature procedures. $\mathrm{CH}_{2} \mathrm{Cl}_{2}$ was dried and degassed using a PureSolv solvent purification system (Innovative Technology Inc.). Thin-layer chromatography was conducted on Merck 60 F254 
TLC silica-gel-coated aluminum sheets and verified by a UV lamp at $254 \mathrm{~nm}$ and $\mathrm{KMnO}_{4}$ staining. Purification of the ligands was achieved via a manual chromatograph using silica gel (Silicycle R12030B) or a Varian 971-FP flash chromatography system using prepackaged silica gel columns (Luknova).

Instrumentation and Methods. ${ }^{1} \mathrm{H}(400 \mathrm{MHz}),{ }^{13} \mathrm{C}(101 \mathrm{MHz})$, and ${ }^{31} \mathrm{P}(162 \mathrm{MHz}) \mathrm{NMR}$ spectra were recorded on a Bruker Avance II 400 spectrometer at $298 \mathrm{~K}$. Chemical shifts are reported in parts per million and referenced to deuterated solvent residual peaks $\left(\mathrm{CDCl}_{3}\right.$ : $\left.{ }^{1} \mathrm{H}, \delta 7.26 \mathrm{ppm} ;{ }^{13} \mathrm{C}\left\{{ }^{1} \mathrm{H}\right\}, \delta 77.16 \mathrm{ppm}\right)$. Coupling constants $(J)$ are reported in hertz. High-resolution ESI-MS spectra were obtained on a Thermo-Finnigan LCQ Deca XP Plus quadropole ion-trap instrument operated in positive-ion or negative-ion mode. Elemental analyses were carried out by the microanalytical laboratory at EPFL using a Thermo Scientific Flash 2000 organic elemental analyzer. UV-vis spectra were recorded using a SpectroMax M5e multimode microplate reader (using SoftMax Pro software, version 6.2.2). The diffraction data of compounds $\mathbf{9 b}$ and $9 \mathrm{c}$ were measured at low temperature [100(2) K] using Mo K $\alpha$ radiation on a Bruker APEX II CCD diffractometer equipped with a Kappa geometry goniometer. The data sets were reduced by EvalCCD ${ }^{79}$ and then corrected for absorption. ${ }^{80}$ The solutions and refinements were performed by SHELX.81,82 The crystal structures were refined using full-matrix least squares based on $F^{2}$, with all non-hydrogen atoms anisotropically defined. Hydrogen atoms were placed in calculated positions by means of the "riding" model. The $\log P$ values of PEG and alkyl linker compounds were predicted using the Virtual Computational Chemistry Lab (VCCLAB). ${ }^{75,76}$ The experimental $\log P_{\text {octanol } / \mathrm{H}_{2} \mathrm{O}}$ values were determined using the shake-flask method, ${ }^{77}$ and the absorbance of each fraction was recorded using a SpectroMax M5e multimode microplate reader (using SoftMax Pro software, version 6.2.2). The absorbance of the MTT assay 96-well plates was recorded using a SpectroMax M5e multimode microplate reader (using SoftMax Pro software, version 6.2.2)

Synthesis. General Procedure for the Synthesis of the Diphosphine Ligands 1a-7a. 4-(Diphenylphosphanyl)benzoic acid (2.2 equiv) and $\mathrm{EDCI}$ (2.4 equiv) were dissolved in $\mathrm{CH}_{2} \mathrm{Cl}_{2}(50 \mathrm{~mL})$ and stirred under $\mathrm{N}_{2}$ at room temperature (RT) for $15 \mathrm{~min}$. The appropriate ethylene glycol (1.0 equiv) and DMAP ( 0.4 equiv) were added, and the mixture was further stirred for $24 \mathrm{~h}$ at $\mathrm{RT}$. The reaction mixture was washed with $\mathrm{H}_{2} \mathrm{O}(150 \mathrm{~mL})$, and the aqueous phase was reextracted with $\mathrm{CH}_{2} \mathrm{Cl}_{2}(2 \times 100 \mathrm{~mL})$. The organic phase was washed with brine $(150 \mathrm{~mL})$, dried over anhydrous sodium sulfate, filtered, and concentrated under reduced pressure. The treated product was purified by flash column chromatography using an adapted elution system of hexanes/ethyl acetate or $\mathrm{CH}_{2} \mathrm{Cl}_{2} /$ methanol. The product was recovered as a colorless viscous solid.

Compound 1a. According to the general procedure, 4(diphenylphosphanyl)benzoic acid (0.900 g, $2.938 \mathrm{mmol}, 2.2$ equiv), mono(ethylene glycol) $(0.075 \mathrm{~mL}, 1.345 \mathrm{mmol}, 1$ equiv), EDCI (0.614 g, $3.203 \mathrm{mmol}, 2.4$ equiv), and DMAP $(0.065 \mathrm{~g}, 0.532 \mathrm{mmol}$, 0.4 equiv) were stirred for $24 \mathrm{~h}$ in $\mathrm{CH}_{2} \mathrm{Cl}_{2}(50 \mathrm{~mL})$. Yield: $0.216 \mathrm{~g}$, $0.338 \mathrm{mmol}, 25 \%$. Elem anal. Calcd for $\mathrm{C}_{40} \mathrm{H}_{32} \mathrm{O}_{4} \mathrm{P}_{2}$ : C, 75.23; $\mathrm{H}, 5.05$. Found: C, 75.55; H, 5.23. ${ }^{1} \mathrm{H}$ NMR $\left(\mathrm{CDCl}_{3}\right): \delta_{\mathrm{H}} 7.97(4 \mathrm{H}, \mathrm{m}$, $\left.4 \mathrm{O}(\mathrm{C}=\mathrm{O})(A r) \mathrm{CCHCHCP},{ }^{3} J_{\mathrm{H}, \mathrm{H}}=8.6 \mathrm{~Hz},{ }^{4} J_{\mathrm{H}, \mathrm{H}}=1.4 \mathrm{~Hz}\right), 7.29-$ $7.40(24 \mathrm{H}, \mathrm{m}, 4 \mathrm{O}(\mathrm{C}=\mathrm{O})(A r) \mathrm{CCHCHCP}, 8 \mathrm{P}(A r) \mathrm{CCHCHCH}$, $8 \mathrm{P}(\mathrm{Ar}) \mathrm{CCHCHCH}, 4 \mathrm{P}(\mathrm{Ar}) \mathrm{CCHCHCH}), 4.64(4 \mathrm{H}, \mathrm{s}, \operatorname{Ar}(\mathrm{C}=$ O) $\left.\mathrm{O}\left(\mathrm{CH}_{2}\right)_{2} \mathrm{O}\right) .{ }^{31} \mathrm{P}$ NMR $\left(\mathrm{CDCl}_{3}\right): \delta_{\mathrm{p}}-4.99(2 \mathrm{P}) .{ }^{13} \mathrm{C}$ NMR $\left(\mathrm{CDCl}_{3}\right): \delta_{\mathrm{C}} 166.3(2 \mathrm{C}, 2 \mathrm{O}(\mathrm{C}=\mathrm{O})(\mathrm{Ar}) \mathrm{CCHCHCP}), 144.6(2 \mathrm{C}, \mathrm{d}$, $\left.2 \mathrm{O}(\mathrm{C}=\mathrm{O})(A r) \mathrm{CCHCHCP},{ }^{1} J_{\mathrm{C}, \mathrm{P}}=15 \mathrm{~Hz}\right), 136.3(4 \mathrm{C}, \mathrm{d}, 4 \mathrm{P}(A r)$ $\left.\mathrm{CCHCHCH},{ }^{1} J_{\mathrm{C}, \mathrm{P}}=11 \mathrm{~Hz}\right), 134.2\left(8 \mathrm{C}, \mathrm{d}, 8 \mathrm{P}(\mathrm{Ar}) \mathrm{CCHCHCH},{ }^{2} J_{\mathrm{C}, \mathrm{P}}=\right.$ $20 \mathrm{~Hz}), 133.3\left(4 \mathrm{C}, \mathrm{d}, 4 \mathrm{O}(\mathrm{C}=\mathrm{O})(\mathrm{Ar}) \mathrm{CCHCHCP},{ }^{2} \mathrm{~J}_{\mathrm{C}, \mathrm{P}}=19 \mathrm{~Hz}\right)$, $129.8(2 \mathrm{C}, 2 \mathrm{O}(\mathrm{C}=\mathrm{O})(\mathrm{Ar}) \mathrm{CCHCHCP}), 129.5(4 \mathrm{C}, \mathrm{d}, 4 \mathrm{O}(\mathrm{C}=$ $\left.\mathrm{O})(\mathrm{Ar}) \mathrm{CCHCHCP},{ }^{3} J_{\mathrm{C}, \mathrm{P}}=6 \mathrm{~Hz}\right), 129.3(4 \mathrm{C}, 4 \mathrm{P}(\mathrm{Ar}) \mathrm{CCHCHCH})$, $128.8\left(8 \mathrm{C}, \mathrm{d}, 8 \mathrm{P}(\mathrm{Ar}) \mathrm{CCHCHCH},{ }^{3} J_{\mathrm{C}, \mathrm{P}}=7 \mathrm{~Hz}\right), 63.0(2 \mathrm{C}, 2(\mathrm{Ar})(\mathrm{C}=$ $\left.\mathrm{O}) \mathrm{O}\left(\mathrm{CH}_{2}\right)_{2} \mathrm{O}\right)$. ESI-MS(+). Calcd: $m / z$ 639.1854 $\left([\mathrm{M}+\mathrm{H}]^{+}\right.$ $\left.\mathrm{C}_{40} \mathrm{H}_{33} \mathrm{O}_{4} \mathrm{P}_{2}^{+}\right)$. Found: $m / z 639.1853$.

Compound $2 a$. According to the general procedure, 4(diphenylphosphanyl)benzoic acid (0.900 g, $2.938 \mathrm{mmol}, 2.2$ equiv), bis(ethylene glycol) $(0.128 \mathrm{~mL}, 1.349 \mathrm{mmol}, 1$ equiv), EDCI ( $0.614 \mathrm{~g}$, $3.203 \mathrm{mmol}, 2.4$ equiv), and DMAP ( $0.065 \mathrm{~g}, 0.532 \mathrm{mmol}, 0.4$ equiv) were stirred for $24 \mathrm{~h}$ in $\mathrm{CH}_{2} \mathrm{Cl}_{2}(50 \mathrm{~mL})$. Yield: $0.328 \mathrm{~g}, 0.480 \mathrm{mmol}$, $36 \%$. Elem anal. Calcd for $\mathrm{C}_{42} \mathrm{H}_{36} \mathrm{O}_{5} \mathrm{P}_{2}: \mathrm{C}, 73.89 ; \mathrm{H}, 5.32$. Found: $\mathrm{C}$, 73.56; $\mathrm{H}, 5.51 .{ }^{1} \mathrm{H}$ NMR $\left(\mathrm{CDCl}_{3}\right): \delta_{\mathrm{H}} 7.94-7.97(4 \mathrm{H}, \mathrm{m}, 4 \mathrm{O}(\mathrm{C}=$ O) $\left.(\mathrm{Ar}) \mathrm{CCHCHCP},{ }^{3} J_{\mathrm{H}, \mathrm{H}}=8.5 \mathrm{~Hz},{ }^{4} J_{\mathrm{H}, \mathrm{H}}=1.5 \mathrm{~Hz}\right), 7.29-7.39(24 \mathrm{H}$, $\mathrm{m}, \quad 4 \mathrm{O}(\mathrm{C}=\mathrm{O})(A r) \mathrm{CCHCHCP}, 8 \mathrm{P}(A r) \mathrm{CCHCHCH}, 8 \mathrm{P}(A r)-$ $\mathrm{CCHCHCH}, 4 \mathrm{P}(\mathrm{Ar}) \mathrm{CCHCHCH}), 4.46-4.49(4 \mathrm{H}, \mathrm{m}, 2 \mathrm{Ar}(\mathrm{C}=$ O) $\left.\mathrm{OCH}_{2} \mathrm{CH}_{2} \mathrm{O}\right), 3.82-3.87\left(4 \mathrm{H}, \mathrm{m}, 2 \mathrm{Ar}(\mathrm{C}=\mathrm{O}) \mathrm{OCH}_{2} \mathrm{CH}_{2} \mathrm{O}\right) .{ }^{31} \mathrm{P}$ $\mathrm{NMR}\left(\mathrm{CDCl}_{3}\right): \delta_{\mathrm{P}}-5.08(2 \mathrm{P}) .{ }^{13} \mathrm{C}$ NMR $\left(\mathrm{CDCl}_{3}\right): \delta_{\mathrm{C}} 166.4(2 \mathrm{C}$, $2 \mathrm{O}(\mathrm{C}=\mathrm{O})(A r) \mathrm{CCHCHCP}), 144.3$ (2C, d, $2 \mathrm{O}(\mathrm{C}=\mathrm{O})(A r)$ $\left.\mathrm{CCHCHCP},{ }^{1} J_{\mathrm{C}, \mathrm{P}}=14 \mathrm{~Hz}\right), 136.3\left(4 \mathrm{C}, \mathrm{d}, 4 \mathrm{P}(\mathrm{Ar}) \mathrm{CCHCHCH},{ }^{1} J_{\mathrm{C}, \mathrm{P}}\right.$ $=11 \mathrm{~Hz}), 134.1\left(8 \mathrm{C}, \mathrm{d}, 8 \mathrm{P}(\mathrm{Ar}) \mathrm{CCHCHCH},{ }^{2} \mathrm{~J}_{\mathrm{C}, \mathrm{P}}=20 \mathrm{~Hz}\right), 133.2$ $\left(4 \mathrm{C}, \mathrm{d}, 4 \mathrm{O}(\mathrm{C}=\mathrm{O})(\mathrm{Ar}) \mathrm{CCHCHCP},{ }^{2} J_{\mathrm{C}, \mathrm{P}}=19 \mathrm{~Hz}\right), 130.0(2 \mathrm{C}$, $2 \mathrm{O}(\mathrm{C}=\mathrm{O})(A r) C \mathrm{CHCHCP}), \quad 129.5(4 \mathrm{C}, \mathrm{d}, 4 \mathrm{O}(\mathrm{C}=\mathrm{O})(\mathrm{Ar})-$ $\left.\mathrm{CCHCHCP},{ }^{3} J_{\mathrm{C}, \mathrm{P}}=6 \mathrm{~Hz}\right), 129.3(4 \mathrm{C}, 4 \mathrm{P}(\mathrm{Ar}) \mathrm{CCHCHCH}), 128.8$ $\left(8 \mathrm{C}, \mathrm{d}, 8 \mathrm{P}(A r) \mathrm{CCHCHCH},{ }^{3} J_{\mathrm{C}, \mathrm{P}}=7 \mathrm{~Hz}\right), 69.3(2 \mathrm{C}, 2(A r)(\mathrm{C}=$ O) $\left.\mathrm{OCH}_{2} \mathrm{CH}_{2} \mathrm{O}\right), 64.2\left(2 \mathrm{C}, 2(\mathrm{Ar})(\mathrm{C}=\mathrm{O}) \mathrm{OCH}_{2} \mathrm{CH}_{2} \mathrm{O}\right)$. ESI-MS(+). Calcd for $\mathrm{C}_{42} \mathrm{H}_{37} \mathrm{O}_{5} \mathrm{P}_{2}^{+}: \mathrm{m} / z 682.6925\left([\mathrm{M}+\mathrm{H}]^{+}\right)$. Found: $\mathrm{m} / z$ 683.2112. Calcd for $\mathrm{C}_{42} \mathrm{H}_{36} \mathrm{NaO}_{5} \mathrm{P}_{2}^{+}$: $705.1931\left([\mathrm{M}+\mathrm{Na}]^{+}\right)$. Found: $\mathrm{m} / \mathrm{z} 705.1936$

Compound 3a. According to the general procedure, 4(diphenylphosphanyl)benzoic acid $(0.900 \mathrm{~g}, 2.938 \mathrm{mmol}, 2.2$ equiv), tris(ethylene glycol) $(0.179 \mathrm{~mL}, 1.340 \mathrm{mmol}, 1$ equiv), EDCI $(0.614 \mathrm{~g}$, $3.203 \mathrm{mmol}, 2.4$ equiv), and DMAP ( $0.065 \mathrm{~g}, 0.532 \mathrm{mmol}, 0.4$ equiv) were stirred for $24 \mathrm{~h}$ in $\mathrm{CH}_{2} \mathrm{Cl}_{2}(50 \mathrm{~mL})$. Yield: $0.446 \mathrm{~g}, 0.614 \mathrm{mmol}$, 46\%. Elem anal. Calcd for $\mathrm{C}_{44} \mathrm{H}_{40} \mathrm{O}_{6} \mathrm{P}_{2}: \mathrm{C}, 72.72 ; \mathrm{H}, 5.55$. Found: $\mathrm{C}$, 72.54; $\mathrm{H}, 5.58 .{ }^{1} \mathrm{H}$ NMR $\left(\mathrm{CDCl}_{3}\right): \delta_{\mathrm{H}} 7.95-7.98(4 \mathrm{H}, \mathrm{m}, 4 \mathrm{O}(\mathrm{C}=$ O) $\left.(A r) \mathrm{CCHCHCP},{ }^{3} J_{\mathrm{H}, \mathrm{H}}=8.4 \mathrm{~Hz},{ }^{4} J_{\mathrm{H}, \mathrm{H}}=1.4 \mathrm{~Hz}\right), 7.29-7.38(24 \mathrm{H}$, $\mathrm{m}, 4 \mathrm{O}(\mathrm{C}=\mathrm{O})(A r) \mathrm{CCHCHCP}, 8 \mathrm{P}(A r) \mathrm{CCHCHCH}, 8 \mathrm{P}(A r)-$ $\mathrm{CCHCHCH}, \quad 4 \mathrm{P}(\mathrm{Ar}) \mathrm{CCHCHCH}), 4.43-4.45(4 \mathrm{H}, \mathrm{m}, 2 \mathrm{Ar}(\mathrm{C}=$ O) $\left.\mathrm{OCH}_{2} \mathrm{CH}_{2} \mathrm{O}\right), 3.79-3.82\left(4 \mathrm{H}, \mathrm{m}, 2 \mathrm{Ar}(\mathrm{C}=\mathrm{O}) \mathrm{OCH}_{2} \mathrm{CH}_{2} \mathrm{O}\right), 3.69$ $\left(4 \mathrm{H}, \mathrm{s}, 2 \mathrm{Ar}(\mathrm{C}=\mathrm{O}) \mathrm{O}\left(\mathrm{CH}_{2}\right)_{2} \mathrm{OCH}_{2}\right) .{ }^{31} \mathrm{P}$ NMR $\left(\mathrm{CDCl}_{3}\right): \delta_{\mathrm{p}}-5.07$ $(2 \mathrm{P}) .{ }^{13} \mathrm{C}$ NMR $\left(\mathrm{CDCl}_{3}\right): \delta_{\mathrm{C}} 166.4 \quad(2 \mathrm{C}, 2 \mathrm{O}(\mathrm{C}=\mathrm{O})(\mathrm{Ar})-$ CCHCHCP $), 144.3\left(2 \mathrm{C}, \mathrm{d}, 2 \mathrm{O}(\mathrm{C}=\mathrm{O})(\mathrm{Ar}) \mathrm{CCHCHCP},{ }^{1} J_{\mathrm{C}, \mathrm{P}}=14\right.$ $\mathrm{Hz}), 136.3\left(4 \mathrm{C}, \mathrm{d}, 4 \mathrm{P}(\mathrm{Ar}) \mathrm{CCHCHCH},{ }^{1} J_{\mathrm{C}, \mathrm{P}}=11 \mathrm{~Hz}\right), 134.1(8 \mathrm{C}, \mathrm{d}$, $\left.8 \mathrm{P}(\mathrm{Ar}) \mathrm{CCHCHCH},{ }^{2} \mathrm{~J}_{\mathrm{CP}}=20 \mathrm{~Hz}\right), 133.3(4 \mathrm{C}, \mathrm{d}, 4 \mathrm{O}(\mathrm{C}=\mathrm{O})(\mathrm{Ar})-$ $\left.\mathrm{CCHCHCP},{ }^{2} J_{\mathrm{C}, \mathrm{P}}=19 \mathrm{~Hz}\right), 130.1(2 \mathrm{C}, 2 \mathrm{O}(\mathrm{C}=\mathrm{O})(A r) \mathrm{CCHCHCP})$, $129.5\left(4 \mathrm{C}, \mathrm{d}, 4 \mathrm{O}(\mathrm{C}=\mathrm{O})(\mathrm{Ar}) \mathrm{CCHCHCP},{ }^{3} J_{\mathrm{CP}}=6 \mathrm{~Hz}\right), 129.3(4 \mathrm{C}$, $4 \mathrm{P}(\mathrm{Ar}) \mathrm{CCHCHCH}), 128.8\left(8 \mathrm{C}, \mathrm{d}, 8 \mathrm{P}(\mathrm{Ar}) \mathrm{CCHCHCH},{ }^{3} J_{\mathrm{C}, \mathrm{P}}=7 \mathrm{~Hz}\right)$, $70.9\left(2 \mathrm{C}, 2(\mathrm{Ar})(\mathrm{C}=\mathrm{O}) \mathrm{O}\left(\mathrm{CH}_{2}\right)_{2} \mathrm{OCH}_{2}\right), 69.4(2 \mathrm{C}, 2(\mathrm{Ar})(\mathrm{C}=$ O) $\left.\mathrm{OCH}_{2} \mathrm{CH}_{2} \mathrm{O}\right), 64.3\left(2 \mathrm{C}, 2(\mathrm{Ar})(\mathrm{C}=\mathrm{O}) \mathrm{OCH}_{2} \mathrm{CH}_{2} \mathrm{O}\right)$. ESI-MS(+). Calcd for $\mathrm{C}_{44} \mathrm{H}_{41} \mathrm{O}_{6} \mathrm{P}_{2}^{+}: m / z 727.2378\left([\mathrm{M}+\mathrm{Na}]^{+}\right)$. Found: $\mathrm{m} / z$ 727.2383. Calcd for $\mathrm{C}_{44} \mathrm{H}_{40} \mathrm{NaO}_{5} \mathrm{P}_{2}^{+}: m / z 749.2198\left([\mathrm{M}+\mathrm{H}]^{+}\right)$. Found: $m / z 749.2202$

Compound 4a. According to the general procedure, 4(diphenylphosphanyl)benzoic acid $(0.900 \mathrm{~g}, 2.938 \mathrm{mmol}, 2.2$ equiv), tetrakis(ethylene glycol) $(0.231 \mathrm{~mL}, 1.338 \mathrm{mmol}, 1$ equiv), EDCI $(0.615 \mathrm{~g}, 3.203,2.4$ equiv), and DMAP $(0.065 \mathrm{~g}, 0.532 \mathrm{mmol}, 0.4$ equiv) were stirred for $24 \mathrm{~h}$ in $\mathrm{CH}_{2} \mathrm{Cl}_{2}(50 \mathrm{~mL})$. Yield: $0.487 \mathrm{~g}, 0.632$ mmol, 47\%. Elem anal. Calcd for $\mathrm{C}_{46} \mathrm{H}_{44} \mathrm{O}_{7} \mathrm{P}_{2}: \mathrm{C}, 71.68 ; \mathrm{H}, 5.75$. Found: C, $71.59, \mathrm{H}, 5.83 .{ }^{1} \mathrm{H}$ NMR $\left(\mathrm{CDCl}_{3}\right): \delta_{\mathrm{H}} 7.95-8.00(4 \mathrm{H}, \mathrm{m}$, $\left.4 \mathrm{O}(\mathrm{C}=\mathrm{O})(\mathrm{Ar}) \mathrm{CCHCHCP},{ }^{3} \mathrm{~J}_{\mathrm{H}, \mathrm{H}}=8.2 \mathrm{~Hz},{ }^{4} J_{\mathrm{H}, \mathrm{H}}=1.6 \mathrm{~Hz}\right), 7.29-$ $7.38(24 \mathrm{H}, \mathrm{m}, 4 \mathrm{O}(\mathrm{C}=\mathrm{O})(\mathrm{Ar}) \mathrm{CCHCHCP}, 8 \mathrm{P}(\mathrm{Ar}) \mathrm{CCHCHCH}$, $8 \mathrm{P}(A r) \mathrm{CCHCHCH}, 4 \mathrm{P}(A r) \mathrm{CCHCHCH}), 4.43-4.46(4 \mathrm{H}, \mathrm{m}$, $\left.2 \mathrm{Ar}(\mathrm{C}=\mathrm{O}) \mathrm{OCH}_{2} \mathrm{CH}_{2} \mathrm{O}\right), 3.77-3.80(4 \mathrm{H}, \quad \mathrm{m}, \quad 2 \mathrm{Ar}(\mathrm{C}=\mathrm{O})-$ $\left.\mathrm{OCH}_{2} \mathrm{CH}_{2} \mathrm{O}\right), 3.61-3.70\left(8 \mathrm{H}, \mathrm{m}, 2 \mathrm{Ar}(\mathrm{C}=\mathrm{O}) \mathrm{O}\left(\mathrm{CH}_{2}\right)_{2} \mathrm{O}\left(\mathrm{CH}_{2}\right)_{2}\right)$. ${ }^{31} \mathrm{P}$ NMR $\left(\mathrm{CDCl}_{3}\right): \delta_{\mathrm{P}}-5.06(2 \mathrm{P}) .{ }^{13} \mathrm{C} \mathrm{NMR}\left(\mathrm{CDCl}_{3}\right): \delta_{\mathrm{C}} 166.4(2 \mathrm{C}$, $2 \mathrm{O}(\mathrm{C}=\mathrm{O})(\mathrm{Ar}) \mathrm{CCHCHCP}), 144.3(2 \mathrm{C}, \mathrm{d}, 2 \mathrm{O}(\mathrm{C}=\mathrm{O})(A r)-$ $\left.\mathrm{CCHCHCP},{ }^{1} J_{\mathrm{C}, \mathrm{P}}=14 \mathrm{~Hz}\right), 136.3\left(4 \mathrm{C}, \mathrm{d}, 4 \mathrm{P}(A r) \mathrm{CCHCHCH},{ }^{1} J_{\mathrm{C}, \mathrm{P}}\right.$ $=11 \mathrm{~Hz}), 134.1\left(8 \mathrm{C}, \mathrm{d}, 8 \mathrm{P}(\mathrm{Ar}) \mathrm{CCHCHCH},{ }^{2} J_{\mathrm{C}, \mathrm{P}}=20 \mathrm{~Hz}\right), 133.3$ $\left(4 \mathrm{C}, \mathrm{d}, 4 \mathrm{O}(\mathrm{C}=\mathrm{O})(\mathrm{Ar}) \mathrm{CCHCHCP},{ }^{2} J_{\mathrm{C}, \mathrm{P}}=19 \mathrm{~Hz}\right), 130.2(2 \mathrm{C}$, $2 \mathrm{O}(\mathrm{C}=\mathrm{O})(\mathrm{Ar}) \mathrm{CCHCHCP}), \quad 129.5 \quad(4 \mathrm{C}, \mathrm{d}, \quad 4 \mathrm{O}(\mathrm{C}=\mathrm{O})(\mathrm{Ar})$ $\left.\mathrm{CCHCHCP},{ }^{3} \mathrm{Cp}=7 \mathrm{~Hz}\right), 129.3(4 \mathrm{C}, 4 \mathrm{P}(\mathrm{Ar}) \mathrm{CCHCHCH}), 128.8$ (8C, d, 8P $\left.(A r) \mathrm{CCHCHCH},{ }^{3} J_{\mathrm{C}, \mathrm{P}}=7 \mathrm{~Hz}\right), 70.77,70.79,70.83(4 \mathrm{C}$, $\left.2(\mathrm{Ar})(\mathrm{C}=\mathrm{O}) \mathrm{O}\left(\mathrm{CH}_{2}\right)_{2} \mathrm{OCH}_{2}, 2(\mathrm{Ar})(\mathrm{C}=\mathrm{O}) \mathrm{O}\left(\mathrm{CH}_{2}\right)_{2} \mathrm{OCH}_{2} \mathrm{CH}_{2}\right)$, $69.3\left(2 \mathrm{C}, 2(\mathrm{Ar})(\mathrm{C}=\mathrm{O}) \mathrm{OCH}_{2} \mathrm{CH}_{2} \mathrm{O}\right), 64.3(2 \mathrm{C}, 2(\mathrm{Ar})(\mathrm{C}=\mathrm{O})-$ $\left.\mathrm{OCH}_{2} \mathrm{CH}_{2} \mathrm{O}\right)$. ESI-MS(+). Calcd for $\mathrm{C}_{46} \mathrm{H}_{45} \mathrm{O}_{7} \mathrm{P}_{2}^{+}: \mathrm{m} / z$ 771.2641 $\left([\mathrm{M}+\mathrm{H}]^{+}\right)$. Found: $m / z$ 771.2630. Calcd for $\mathrm{C}_{46} \mathrm{H}_{44} \mathrm{NaO}_{7} \mathrm{P}_{2}^{+}: \mathrm{m} / z$ $793.2460\left([\mathrm{M}+\mathrm{Na}]^{+}\right)$. Found: $m / z$ 793.2451. 
Compound 5a. According to the general procedure, 4(diphenylphosphanyl)benzoic acid $(0.800 \mathrm{~g}, 2.612 \mathrm{mmol}, 2.2$ equiv), pentakis(ethylene glycol) $(0.251 \mathrm{~mL}, 1.186 \mathrm{mmol}, 1$ equiv), EDCI ( $0.546 \mathrm{~g}, 2.848 \mathrm{mmol}, 2.4$ equiv), and DMAP $(0.058 \mathrm{~g}, 0.475 \mathrm{mmol}$, 0.4 equiv) were stirred for $24 \mathrm{~h}$ in $\mathrm{CH}_{2} \mathrm{Cl}_{2}(50 \mathrm{~mL})$. Yield: $0.621 \mathrm{~g}$, $0.762 \mathrm{mmol}, 64 \%$. Elem anal. Calcd for $\mathrm{C}_{48} \mathrm{H}_{48} \mathrm{O}_{8} \mathrm{P}_{2}: \mathrm{C}, 70.75 ; \mathrm{H}, 5.94$. Found: C, 70.85, H, 6.04. ${ }^{1} \mathrm{H}$ NMR $\left(\mathrm{CDCl}_{3}\right): \delta_{\mathrm{H}} 7.96-7.99(4 \mathrm{H}, \mathrm{m}$, $\left.4 \mathrm{O}(\mathrm{C}=\mathrm{O})(\mathrm{Ar}) \mathrm{CCHCHCP},{ }^{3} \mathrm{~J}_{\mathrm{H}, \mathrm{H}}=8.4 \mathrm{~Hz},{ }^{4} \mathrm{~J}_{\mathrm{H}, \mathrm{H}}=1.6 \mathrm{~Hz}\right), 7.28-$ $7.37(24 \mathrm{H}, \mathrm{m}, 4 \mathrm{O}(\mathrm{C}=\mathrm{O})(\mathrm{Ar}) \mathrm{CCHCHCP}, 8 \mathrm{P}(\mathrm{Ar}) \mathrm{CCHCHCH}$, $8 \mathrm{P}(A r) \mathrm{CCHCHCH}, 4 \mathrm{P}(A r) \mathrm{CCHCHCH}), 4.44-4.46$ (4H, m, $\left.2 A r(\mathrm{C}=\mathrm{O}) \mathrm{OCH}_{2} \mathrm{CH}_{2} \mathrm{O}\right), 3.79-3.81(4 \mathrm{H}, \mathrm{m}, 2 \operatorname{Ar}(\mathrm{C}=\mathrm{O})-$ $\left.\mathrm{OCH}_{2} \mathrm{CH}_{2} \mathrm{O}\right), 3.65-3.68\left(4 \mathrm{H}, \mathrm{m}, 2 \mathrm{Ar}(\mathrm{C}=\mathrm{O}) \mathrm{O}\left(\mathrm{CH}_{2}\right)_{2} \mathrm{OCH}_{2} \mathrm{CH}_{2}\right)$, 3.61-3.64 (4H, m, $\left.2 A r(\mathrm{C}=\mathrm{O}) \mathrm{O}\left(\mathrm{CH}_{2}\right)_{2} \mathrm{OCH}_{2} \mathrm{CH}_{2}\right), 3.62(4 \mathrm{H}, \mathrm{s}$, $\left.2 \mathrm{Ar}(\mathrm{C}=\mathrm{O}) \mathrm{O}\left(\mathrm{CH}_{2}\right)_{2} \mathrm{O}\left(\mathrm{CH}_{2}\right)_{2} \mathrm{OCH}_{2}\right),{ }^{31} \mathrm{P} \mathrm{NMR}\left(\mathrm{CDCl}_{3}\right): \delta_{\mathrm{p}}-5.08$ (2P). ${ }^{13} \mathrm{C}$ NMR $\left(\mathrm{CDCl}_{3}\right): \delta_{\mathrm{C}} 166.4(2 \mathrm{C}, 2 \mathrm{O}(\mathrm{C}=\mathrm{O})(\mathrm{Ar})-$ $\mathrm{CCHCHCP}), 144.3\left(2 \mathrm{C}, \mathrm{d}, 2 \mathrm{O}(\mathrm{C}=\mathrm{O})(\mathrm{Ar}) \mathrm{CCHCHCP},{ }^{1} J_{\mathrm{C}, \mathrm{P}}=14\right.$ $\mathrm{Hz}), 136.3\left(4 \mathrm{C}, \mathrm{d}, 4 \mathrm{P}(\mathrm{Ar}) \mathrm{CCHCHCH},{ }^{1} J_{\mathrm{C}, \mathrm{P}}=11 \mathrm{~Hz}\right), 134.1(8 \mathrm{C}, \mathrm{d}$, $\left.8 \mathrm{P}(\mathrm{Ar}) \mathrm{CCHCHCH},{ }^{2} \mathrm{~J}_{\mathrm{C}, \mathrm{P}}=20 \mathrm{~Hz}\right), 133.2(4 \mathrm{C}, \mathrm{d}, 4 \mathrm{O}(\mathrm{C}=\mathrm{O})(\mathrm{Ar})-$ $\left.\mathrm{CCHCHCP},{ }^{2} \mathrm{~J}_{\mathrm{C}, \mathrm{P}}=19 \mathrm{~Hz}\right), 130.1(2 \mathrm{C}, 2 \mathrm{O}(\mathrm{C}=\mathrm{O})(\mathrm{Ar}) \mathrm{CCHCHCP})$, $129.5\left(4 \mathrm{C}, \mathrm{d}, 4 \mathrm{O}(\mathrm{C}=\mathrm{O})(\mathrm{Ar}) \mathrm{CCHCHCP},{ }^{3} \mathrm{C}_{\mathrm{C}, \mathrm{P}}=6 \mathrm{~Hz}\right), 129.1(4 \mathrm{C}$, $4 \mathrm{P}(\mathrm{Ar}) \mathrm{CCHCHCH}), 128.8\left(8 \mathrm{C}, \mathrm{d}, 8 \mathrm{P}(\mathrm{Ar}) \mathrm{CCHCHCH},{ }^{3} J_{\mathrm{C}, \mathrm{P}}=7 \mathrm{~Hz}\right)$, 70.80, 70.76, $70.73\left(6 \mathrm{C}, 2(\mathrm{Ar})(\mathrm{C}=\mathrm{O}) \mathrm{O}\left(\mathrm{CH}_{2}\right)_{2} \mathrm{OCH}_{2}, 2(\mathrm{Ar})(\mathrm{C}=\right.$ O)O $\left.\left(\mathrm{CH}_{2}\right)_{2} \mathrm{OCH}_{2} \mathrm{CH}_{2}, 2(\mathrm{Ar})(\mathrm{C}=\mathrm{O}) \mathrm{O}\left(\mathrm{CH}_{2}\right)_{2} \mathrm{O}\left(\mathrm{CH}_{2}\right)_{2} \mathrm{OCH}_{2}\right), 69.3$ $\left(2 \mathrm{C}, 2(\mathrm{Ar})(\mathrm{C}=\mathrm{O}) \mathrm{OCH}_{2} \mathrm{CH}_{2} \mathrm{O}\right), \quad 64.3 \quad(2 \mathrm{C}, 2(\mathrm{Ar})(\mathrm{C}=\mathrm{O})-$ $\left.\mathrm{OCH}_{2} \mathrm{CH}_{2} \mathrm{O}\right)$. ESI-MS(+). Calcd for $\mathrm{C}_{48} \mathrm{H}_{49} \mathrm{O}_{8} \mathrm{P}_{2}^{+}: \mathrm{m} / z$ 815.2897 $\left([\mathrm{M}+\mathrm{H}]^{+}\right)$. Found: $m / z$ 815.2905. Calcd for $\mathrm{C}_{48} \mathrm{H}_{48} \mathrm{NaO}_{8} \mathrm{P}_{2}^{+}: \mathrm{m} / z$ 837.2722 $\left([\mathrm{M}+\mathrm{Na}]^{+}\right)$. Found: $m / z$ 837.2723.

Compound 6a. According to the general procedure, 4(diphenylphosphanyl)benzoic acid $(0.800 \mathrm{~g}, 2.612 \mathrm{mmol}, 2.2$ equiv), hexakis(ethylene glycol) $(0.298 \mathrm{~mL}, 1.190 \mathrm{mmol}, 1$ equiv), EDCI (0.546 g, $2.848 \mathrm{mmol}, 2.4$ equiv), and DMAP (0.058 g, $0.475 \mathrm{mmol}$, 0.4 equiv) were stirred for $24 \mathrm{~h}$ in $\mathrm{CH}_{2} \mathrm{Cl}_{2}(50 \mathrm{~mL})$. Yield: $0.341 \mathrm{~g}$, $0.397 \mathrm{mmol}, 33 \%$. Elem anal. Calcd for $\mathrm{C}_{50} \mathrm{H}_{52} \mathrm{O}_{9} \mathrm{P}_{2}: \mathrm{C}, 69.92 ; \mathrm{H}, 6.10$. Found: C, 70.04; $\mathrm{H}, 6.13 .{ }^{1} \mathrm{H}$ NMR $\left(\mathrm{CDCl}_{3}\right): \delta_{\mathrm{H}} 7.96-7.99(4 \mathrm{H}, \mathrm{m}$, $\left.4 \mathrm{O}(\mathrm{C}=\mathrm{O})(\mathrm{Ar}) \mathrm{CCHCHCP},{ }^{3} J_{\mathrm{H}, \mathrm{H}}=8.4 \mathrm{~Hz},{ }^{4} \mathrm{~J}_{\mathrm{H}, \mathrm{H}}=1.2 \mathrm{~Hz}\right), 7.28-$ $7.39(24 \mathrm{H}, \mathrm{m}, 4 \mathrm{O}(\mathrm{C}=\mathrm{O})(\mathrm{Ar}) \mathrm{CCHCHCP}, 8 \mathrm{P}(A r) \mathrm{CCHCHCH}$, $8 \mathrm{P}(A r) \mathrm{CCHCH}-\mathrm{CH}, 4 \mathrm{P}(A r) \mathrm{CCHCHCH}), 4.44-4.47$ (4H, m, $\left.2 \operatorname{Ar}(\mathrm{C}=\mathrm{O}) \mathrm{OCH}_{2} \mathrm{CH}_{2} \mathrm{O}\right), 3.79-3.82(4 \mathrm{H}, \quad \mathrm{m}, 2 \operatorname{Ar}(\mathrm{C}=\mathrm{O})-$ $\left.\mathrm{OCH}_{2} \mathrm{CH}_{2} \mathrm{O}\right), 3.66-3.69\left(4 \mathrm{H}, \mathrm{m}, 2 \mathrm{Ar}(\mathrm{C}=\mathrm{O}) \mathrm{O}\left(\mathrm{CH}_{2}\right)_{2} \mathrm{OCH}_{2} \mathrm{CH}_{2}\right)$, 3.61-3.65 $\left(4 \mathrm{H}, \mathrm{m}, 2 \mathrm{Ar}(\mathrm{C}=\mathrm{O}) \mathrm{O}\left(\mathrm{CH}_{2}\right)_{2} \mathrm{OCH}_{2} \mathrm{CH}_{2}\right), 3.58-3.62(8 \mathrm{H}$, $\mathrm{m}, 2 \mathrm{Ar}(\mathrm{C}=\mathrm{O}) \mathrm{O}\left(\mathrm{CH}_{2}\right)_{2} \mathrm{O}\left(\mathrm{CH}_{2}\right)_{2} \mathrm{O}\left(\mathrm{CH}_{2}\right)_{2} \cdot{ }^{31} \mathrm{P}$ NMR $\left(\mathrm{CDCl}_{3}\right): \delta_{\mathrm{P}}$ $5.06(2 \mathrm{P}) .{ }^{13} \mathrm{C}$ NMR $\left(\mathrm{CDCl}_{3}\right): \delta_{\mathrm{C}} 166.3(2 \mathrm{C}, 2 \mathrm{O}(\mathrm{C}=\mathrm{O})(\mathrm{Ar})-$ CCHCHCP $), 144.1\left(2 \mathrm{C}, \mathrm{d}, 2 \mathrm{O}(\mathrm{C}=\mathrm{O})(A r) \mathrm{CCHCHCP},{ }^{1} J_{\mathrm{C}, \mathrm{P}}=14\right.$ $\mathrm{Hz}), 136.1\left(4 \mathrm{C}, \mathrm{d}, 4 \mathrm{P}(A r) C \mathrm{CHCHCH},{ }^{1} J_{\mathrm{C}, \mathrm{P}}=11 \mathrm{~Hz}\right), 133.9(8 \mathrm{C}, \mathrm{d}$, $\left.8 \mathrm{P}(\mathrm{Ar}) \mathrm{CCHCHCH},{ }^{2} \mathrm{~J}_{\mathrm{C}, \mathrm{P}}=20 \mathrm{~Hz}\right), 133.1(4 \mathrm{C}, \mathrm{d}, 4 \mathrm{O}(\mathrm{C}=\mathrm{O})(A r)-$ $\left.\mathrm{CCHCHCP},{ }^{2} J_{\mathrm{C}, \mathrm{P}}=19 \mathrm{~Hz}\right), 130.0(2 \mathrm{C}, 2 \mathrm{O}(\mathrm{C}=\mathrm{O})(\mathrm{Ar}) \mathrm{CCHCHCP})$, $129.4\left(4 \mathrm{C}, \mathrm{d}, 4 \mathrm{O}(\mathrm{C}=\mathrm{O})(\mathrm{Ar}) \mathrm{CCHCHCP},{ }^{3} \mathrm{~J}_{\mathrm{C}, \mathrm{P}}=6 \mathrm{~Hz}\right), 129.1(4 \mathrm{C}$, $4 \mathrm{P}(\mathrm{Ar}) \mathrm{CCHCHCH}), 128.7\left(8 \mathrm{C}, \mathrm{d}, 8 \mathrm{P}(\mathrm{Ar}) \mathrm{CCHCHCH},{ }^{3} J_{\mathrm{C}, \mathrm{P}}=7 \mathrm{~Hz}\right)$, 70.70, 70.65, 70.63, $70.59\left(8 \mathrm{C}, 2(\mathrm{Ar})(\mathrm{C}=\mathrm{O}) \mathrm{O}\left(\mathrm{CH}_{2}\right)_{2} \mathrm{OCH}_{2}, 2(\mathrm{Ar})\right.$ $(\mathrm{C}=\mathrm{O}) \mathrm{O}\left(\mathrm{CH}_{2}\right)_{2} \mathrm{OCH}_{2} \mathrm{CH}_{2}, 2(\mathrm{Ar})(\mathrm{C}=\mathrm{O}) \mathrm{O}\left(\mathrm{CH}_{2}\right)_{2} \mathrm{O}\left(\mathrm{CH}_{2}\right)_{2} \mathrm{OCH}_{2}$, $\left.2(\mathrm{Ar})(\mathrm{C}=\mathrm{O}) \mathrm{O}\left(\mathrm{CH}_{2}\right)_{2} \mathrm{O}\left(\mathrm{CH}_{2}\right)_{2} \mathrm{OCH}_{2} \mathrm{CH}_{2}\right), 69.2(2 \mathrm{C}, 2(\mathrm{Ar})(\mathrm{C}=$ O) $\left.\mathrm{OCH}_{2} \mathrm{CH}_{2} \mathrm{O}\right), 64.2\left(2 \mathrm{C}, 2(\mathrm{Ar})(\mathrm{C}=\mathrm{O}) \mathrm{OCH}_{2} \mathrm{CH}_{2} \mathrm{O}\right)$. ESI-MS(+). Calcd for $\mathrm{C}_{50} \mathrm{H}_{53} \mathrm{O}_{9} \mathrm{P}_{2}^{+}: m / z$ 859.3165 $\left([\mathrm{M}+\mathrm{H}]^{+}\right)$. Found: $m / z$ 859.3168. Calcd for $\mathrm{C}_{50} \mathrm{H}_{52} \mathrm{NaO}_{9} \mathrm{P}_{2}^{+}: m / z$ 881.2984 $\left([\mathrm{M}+\mathrm{Na}]^{+}\right)$. Found: $m / z$ 881.2988.

Compound 7a. According to the general procedure, 4(diphenylphosphanyl)benzoic acid $(0.800 \mathrm{~g}, 2.612 \mathrm{mmol}, 2.2$ equiv), octakis(ethylene glycol) $(0.440 \mathrm{~g}, 1.188 \mathrm{mmol}, 1$ equiv), EDCI $(0.546$ g, $2.848 \mathrm{mmol}, 2.4$ equiv), and DMAP (0.058 g, $0.475 \mathrm{mmol}, 0.4$ equiv) were stirred for $24 \mathrm{~h}$ in $\mathrm{CH}_{2} \mathrm{Cl}_{2}(50 \mathrm{~mL})$. Yield: $0.499 \mathrm{~g}, 0.527$ mmol, 45\%. Elem anal. Calcd for $\mathrm{C}_{54} \mathrm{H}_{60} \mathrm{O}_{11} \mathrm{P}_{2}: \mathrm{C}, 68.49 ; \mathrm{H}, 6.39$. Found: $\mathrm{C}, 68.16 ; \mathrm{H}, 6.35 .{ }^{1} \mathrm{H}$ NMR $\left(\mathrm{CDCl}_{3}\right): \delta_{\mathrm{H}} 7.96-8.00(4 \mathrm{H}, \mathrm{m}$, $\left.4 \mathrm{O}(\mathrm{C}=\mathrm{O})(\mathrm{Ar}) \mathrm{CCHCHCP},{ }^{3} J_{\mathrm{H}, \mathrm{H}}=8.4 \mathrm{~Hz},{ }^{4} \mathrm{~J}_{\mathrm{H}, \mathrm{H}}=1.6 \mathrm{~Hz}\right), 7.29-$ $7.37(24 \mathrm{H}, \mathrm{m}, 4 \mathrm{O}(\mathrm{C}=\mathrm{O})(A r) \mathrm{CCHCHCP}, 8 \mathrm{P}(A r) \mathrm{CCHCHCH}$, $8 \mathrm{P}(\mathrm{Ar}) \mathrm{CCHCHCH}, 4 \mathrm{P}(\mathrm{Ar}) \mathrm{CCHCHCH}), 4.45-4.47$ (4H, m, $\left.2 A r(\mathrm{C}=\mathrm{O}) \mathrm{OCH}_{2} \mathrm{CH}_{2} \mathrm{O}\right), 3.80-3.82(4 \mathrm{H}, \quad \mathrm{m}, \quad 2 \operatorname{Ar}(\mathrm{C}=\mathrm{O})-$ $\left.\mathrm{OCH}_{2} \mathrm{CH}_{2} \mathrm{O}\right), 3.67-3.70\left(4 \mathrm{H}, \mathrm{m}, 2 \mathrm{Ar}(\mathrm{C}=\mathrm{O}) \mathrm{O}\left(\mathrm{CH}_{2}\right)_{2} \mathrm{OCH}_{2} \mathrm{CH}_{2}\right)$, 3.63-3.66 (4H, m, $\left.2 \mathrm{Ar}(\mathrm{C}=\mathrm{O}) \mathrm{O}\left(\mathrm{CH}_{2}\right)_{2} \mathrm{OCH}_{2} \mathrm{CH}_{2}\right), 3.60-3.62(8 \mathrm{H}$, m, $2 A r(\mathrm{C}=\mathrm{O}) \mathrm{O}\left(\mathrm{CH}_{2}\right)_{2} \mathrm{O}\left(\mathrm{CH}_{2}\right)_{2} \mathrm{O}\left(\mathrm{CH}_{2}\right)_{2}, 3.61(8 \mathrm{H}, \mathrm{s}, 2 \mathrm{Ar}(\mathrm{C}=$ $\mathrm{O}) \mathrm{O}\left(\mathrm{CH}_{2}\right)_{2} \mathrm{O}\left(\mathrm{CH}_{2}\right)_{2} \mathrm{O}\left(\mathrm{CH}_{2}\right)_{2} \mathrm{O}\left(\mathrm{CH}_{2}\right)_{2} .{ }^{31} \mathrm{P}$ NMR $\left(\mathrm{CDCl}_{3}\right): \delta_{\mathrm{P}}$
$-5.06(2 \mathrm{P}) .{ }^{13} \mathrm{C}$ NMR $\left(\mathrm{CDCl}_{3}\right): \delta_{\mathrm{C}} 166.4(2 \mathrm{C}, 2 \mathrm{O}(\mathrm{C}=\mathrm{O})(\mathrm{Ar})-$ $\mathrm{CCHCHCP}), 144.4\left(2 \mathrm{C}, \mathrm{d}, 2 \mathrm{O}(\mathrm{C}=\mathrm{O})(\mathrm{Ar}) \mathrm{CCHCHCP},{ }^{1} J_{\mathrm{C}, \mathrm{P}}=15\right.$ $\mathrm{Hz}), 136.5\left(4 \mathrm{C}, \mathrm{d}, 4 \mathrm{P}(\mathrm{Ar}) \mathrm{CCHCHCH},{ }^{1} J_{\mathrm{C}, \mathrm{P}}=11 \mathrm{~Hz}\right), 134.1(8 \mathrm{C}, \mathrm{d}$, $\left.8 \mathrm{P}(\mathrm{Ar}) \mathrm{CCHCHCH},{ }^{2} J_{\mathrm{C}, \mathrm{P}}=20 \mathrm{~Hz}\right), 133.3(4 \mathrm{C}, \mathrm{d}, 4 \mathrm{O}(\mathrm{C}=\mathrm{O})(\mathrm{Ar})-$ $\left.\mathrm{CCHCHCP},{ }^{2} J_{\mathrm{C}, \mathrm{P}}=19 \mathrm{~Hz}\right), 130.1(2 \mathrm{C}, 2 \mathrm{O}(\mathrm{C}=\mathrm{O})(\mathrm{Ar}) \mathrm{CCHCHCP})$, $129.5\left(4 \mathrm{C}, \mathrm{d}, 4 \mathrm{O}(\mathrm{C}=\mathrm{O})(\mathrm{Ar}) \mathrm{CCHCHCP},{ }^{3} J_{\mathrm{C}, \mathrm{P}}=6 \mathrm{~Hz}\right), 129.3(4 \mathrm{C}$, $4 \mathrm{P}(\mathrm{Ar}) \mathrm{CCHCHCH}), 128.8\left(8 \mathrm{C}, \mathrm{d}, 8 \mathrm{P}(\mathrm{Ar}) \mathrm{CCHCHCH},{ }^{3} J_{\mathrm{C}, \mathrm{P}}=7 \mathrm{~Hz}\right)$, $70.92,70.88,70.85,70.81 \quad\left(12 \mathrm{C}, 2(\mathrm{Ar})(\mathrm{C}=\mathrm{O}) \mathrm{O}\left(\mathrm{CH}_{2}\right)_{2} \mathrm{OCH}_{2}\right.$, $2(A r)(\mathrm{C}=\mathrm{O}) \mathrm{O}\left(\mathrm{CH}_{2}\right)_{2} \mathrm{OCH}_{2} \mathrm{CH}_{2}, \quad 2(A r)(\mathrm{C}=\mathrm{O}) \mathrm{O}\left(\mathrm{CH}_{2}\right)_{2} \mathrm{O}-$ $\left(\mathrm{CH}_{2}\right)_{2} \mathrm{OCH}_{2}, 2(\mathrm{Ar})(\mathrm{C}=\mathrm{O}) \mathrm{O}\left(\mathrm{CH}_{2}\right)_{2} \mathrm{O}\left(\mathrm{CH}_{2}\right)_{2} \mathrm{OCH}_{2} \mathrm{CH}_{2}, 2(\mathrm{Ar})-$ $(\mathrm{C}=\mathrm{O}) \mathrm{O}\left(\mathrm{CH}_{2}\right)_{2} \mathrm{O}\left(\mathrm{CH}_{2}\right)_{2} \mathrm{O}\left(\mathrm{CH}_{2}\right)_{2} \mathrm{OCH}_{2}, 2(\mathrm{Ar})(\mathrm{C}=\mathrm{O}) \mathrm{O}-$ $\left.\left(\mathrm{CH}_{2}\right)_{2} \mathrm{O}\left(\mathrm{CH}_{2}\right)_{2} \mathrm{O}\left(\mathrm{CH}_{2}\right)_{2} \mathrm{OCH}_{2} \mathrm{CH}_{2}\right), 69.4(2 \mathrm{C}, 2(\mathrm{Ar})(\mathrm{C}=\mathrm{O})-$ $\left.\mathrm{OCH}_{2} \mathrm{CH}_{2} \mathrm{O}\right), 64.7\left(2 \mathrm{C}, 2(\mathrm{Ar})(\mathrm{C}=\mathrm{O}) \mathrm{OCH}_{2} \mathrm{CH}_{2} \mathrm{O}\right)$. ESI-MS(+). Calcd for $\mathrm{C}_{54} \mathrm{H}_{61} \mathrm{O}_{11} \mathrm{P}_{2}^{+}: m / z 947.3684\left([\mathrm{M}+\mathrm{H}]^{+}\right)$. Found: $\mathrm{m} / z$ 947.3671. Calcd for $\mathrm{C}_{54} \mathrm{H}_{60} \mathrm{NaO}_{11} \mathrm{P}_{2}^{+}: m / z 969.3509\left([\mathrm{M}+\mathrm{Na}]^{+}\right)$. Found: $m / z$ 969.3488.

General Procedure for the Synthesis of the Diruthenium Complexes $\mathbf{1 b}-\mathbf{7 b}$. [ $\mathrm{Ru}\left(\eta^{6}-p\right.$-cymene $) \mathrm{Cl}_{2} \mathrm{Cl}_{2}$ (1 equiv) and the appropriate ligand $1 \mathrm{a}-7 \mathrm{a}$ (1 equiv) in $\mathrm{CH}_{2} \mathrm{Cl}_{2}(12 \mathrm{~mL})$ were stirred for $42 \mathrm{~h}$ at $\mathrm{RT}$ under $\mathrm{N}_{2}$ in the dark. The reaction evolution was monitored by ${ }^{1} \mathrm{H}$ and ${ }^{31} \mathrm{P}$ NMR $\left(\mathrm{CDCl}_{3}\right)$. The reaction mixture was concentrated under reduced pressure and then further dried under high vacuum to yield the product as a red solid.

Compound 1b. According to the general procedure, $\left[\mathrm{Ru}\left(\eta^{6}-p\right.\right.$ cymene $) \mathrm{Cl}_{2} \mathrm{Cl}_{2}$ (0.19 g, $0.307 \mathrm{mmol}, 1$ equiv), 1a (0.20 g, 0.307 mmol, 1 equiv), and $\mathrm{CH}_{2} \mathrm{Cl}_{2}(12 \mathrm{~mL})$ were stirred for $42 \mathrm{~h}$ at $\mathrm{RT}$. The product was isolated as a dark-red solid. Yield: $0.376 \mathrm{~g}, 0.301 \mathrm{mmol}$, 98\%. Elem anal. Calcd for $\mathrm{C}_{60} \mathrm{H}_{60} \mathrm{Cl}_{4} \mathrm{O}_{4} \mathrm{P}_{2} \mathrm{Ru}_{2} \cdot \mathrm{CH}_{2} \mathrm{Cl}_{2}$ : C, 54.84; $\mathrm{H}$, 4.68. Found: $\mathrm{C}, 54.77 ; \mathrm{H}, 4.93 . \mathrm{CH}_{2} \mathrm{Cl}_{2}$ originates from the reaction solvent. ${ }^{1} \mathrm{H}$ NMR $\left(\mathrm{CDCl}_{3}\right): \delta_{\mathrm{H}} 7.88-7.97(8 \mathrm{H}, \mathrm{m}, \quad 4 \mathrm{O}(\mathrm{C}=$ $\mathrm{O})(\mathrm{Ar}) \mathrm{CCHCHCP}, 4 \mathrm{O}(\mathrm{C}=\mathrm{O})(\mathrm{Ar}) \mathrm{CCHCHCP}), 7.77-7.82(8 \mathrm{H}$, $\mathrm{m}, 8 \mathrm{P}(\mathrm{Ar}) \mathrm{CCHCHCH}), 7.34-7.44(12 \mathrm{H}, \mathrm{m}, 8 \mathrm{P}(\mathrm{Ar}) \mathrm{CCHCHCH}$, $4 \mathrm{P}(\mathrm{Ar}) \mathrm{CCHCHCH}), 5.20\left(4 \mathrm{H}, \mathrm{d}, 4 \mathrm{CH}_{3}(\mathrm{Ar}) \mathrm{CCHCHC},{ }^{3} J_{\mathrm{H}, \mathrm{H}}=6.1\right.$ $\mathrm{Hz}), 4.98\left(4 \mathrm{H}, \mathrm{d}, 4 \mathrm{CH}_{3}(\mathrm{Ar}) \mathrm{CCHCHC},{ }^{3} \mathrm{~J}_{\mathrm{H}, \mathrm{H}}=6.1 \mathrm{~Hz}\right), 4.58(4 \mathrm{H}, \mathrm{s}$, $\left.2 \mathrm{Ar}(\mathrm{C}=\mathrm{O}) \mathrm{OCH}_{2} \mathrm{CH}_{2} \mathrm{O}\right), 2.83(2 \mathrm{H}$, sept, $2(A r)-$ $\left.\mathrm{CCHCHCCH}\left(\mathrm{CH}_{3}\right)_{2},{ }^{3} \mathrm{~J}_{\mathrm{H}, \mathrm{H}}=7.2 \mathrm{~Hz}\right), 1.84\left(6 \mathrm{H}, \mathrm{s}, 2 \mathrm{CH}_{3}(\mathrm{Ar})-\right.$ $\mathrm{CCHCHC}), 1.09\left(12 \mathrm{H}, \mathrm{d}, 2(\mathrm{Ar}) \mathrm{CCHCHCCH}\left(\mathrm{CH}_{3}\right)_{2},{ }^{3} J_{\mathrm{H}, \mathrm{H}}=7.2\right.$ $\mathrm{Hz}) .{ }^{31} \mathrm{P} \mathrm{NMR}\left(\mathrm{CDCl}_{3}\right): \delta_{\mathrm{P}} 25.00(2 \mathrm{P}) .{ }^{13} \mathrm{C} \mathrm{NMR}\left(\mathrm{CDCl}_{3}\right): \delta_{\mathrm{C}} 165.8$ $(2 \mathrm{C}, 2 \mathrm{O}(\mathrm{C}=\mathrm{O})(\mathrm{Ar}) \mathrm{CCHCHCP}), 139.7(2 \mathrm{C}, \mathrm{d}, 2 \mathrm{O}(\mathrm{C}=\mathrm{O})(A r)-$ CCHCHCP, $\left.{ }^{1} J_{\mathrm{C}, \mathrm{P}}=43 \mathrm{~Hz}\right), 134.4(4 \mathrm{C}, \mathrm{d}, 4 \mathrm{O}(\mathrm{C}=\mathrm{O})(A r)-$ $\left.\mathrm{CCHCHCP},{ }^{2} J_{\mathrm{C}, \mathrm{P}}=9 \mathrm{~Hz}\right), 134.3\left(8 \mathrm{C}, \mathrm{d}, 8 \mathrm{P}(\mathrm{Ar}) \mathrm{CCHCHCH},{ }^{2} J_{\mathrm{C}, \mathrm{P}}\right.$ $=9 \mathrm{~Hz}), 133.3\left(4 \mathrm{C}, \mathrm{d}, 4 \mathrm{P}(\mathrm{Ar}) \mathrm{CCHCHCH},{ }^{1} J_{\mathrm{C}, \mathrm{P}}=45 \mathrm{~Hz}\right), 130.9(2 \mathrm{C}$, d, $\left.2 \mathrm{O}(\mathrm{C}=\mathrm{O})(A r) C \mathrm{CHCHCP},{ }^{4} J_{\mathrm{C}, \mathrm{P}}=3 \mathrm{~Hz}\right), 130.6(4 \mathrm{C}, \mathrm{d}$, $\left.4 \mathrm{P}(\mathrm{Ar}) \mathrm{CCHCHCH},{ }^{4} \mathrm{~J}_{\mathrm{C}, \mathrm{P}}=2 \mathrm{~Hz}\right), 128.8(4 \mathrm{C}, \mathrm{d}, 4 \mathrm{O}(\mathrm{C}=\mathrm{O})(\mathrm{Ar})-$ $\left.\mathrm{CCHCHCP},{ }^{3} J_{\mathrm{C}, \mathrm{P}}=10 \mathrm{~Hz}\right), 128.3\left(8 \mathrm{C}, \mathrm{d}, 8 \mathrm{P}(\mathrm{Ar}) \mathrm{CCHCHCH},{ }^{3} \mathrm{~J}_{\mathrm{C}, \mathrm{P}}=\right.$ $10 \mathrm{~Hz}), 111.46,111.43\left(2 \mathrm{C}, 2 \mathrm{CH}_{3}(\mathrm{Ar}) \mathrm{CCHCHC}\right), 96.4(2 \mathrm{C}$, $\left.2 \mathrm{CH}_{3}(\mathrm{Ar}) \mathrm{CCHCHC}\right), 89.12,89.09\left(4 \mathrm{C}, 4 \mathrm{CH}_{3}(\mathrm{Ar}) \mathrm{CCHCHC}\right)$, $87.39,87.34$ (4C, $\left.4 \mathrm{CH}_{3}(\mathrm{Ar}) \mathrm{CCHCHC}\right), 63.0$ (2C, $2(\mathrm{Ar})(\mathrm{C}=$ O) $\left.\mathrm{OCH}_{2} \mathrm{CH}_{2} \mathrm{O}\right), 30.4\left(2 \mathrm{C}, 2(\mathrm{Ar}) \mathrm{CHCHCCH}\left(\mathrm{CH}_{3}\right)_{2}\right), 21.9$ (4C, $\left.2(\mathrm{Ar}) \mathrm{CHCHCCH}\left(\mathrm{CH}_{3}\right)_{2}\right), 17.9\left(2 \mathrm{C}, 2 \mathrm{CH}_{3}(\mathrm{Ar}) \mathrm{CCHCH}\right)$. ESIMS(+). Calcd for $\mathrm{C}_{60} \mathrm{H}_{60} \mathrm{Cl}_{2} \mathrm{O}_{4} \mathrm{P}_{2} \mathrm{Ru}_{2}{ }^{+}: \mathrm{m} / z 1180.1431$ ([M $\left.2 \mathrm{Cl}]^{+}\right)$. Found: $m / z$ 1180.1499. Calcd for $\mathrm{C}_{60} \mathrm{H}_{60} \mathrm{Cl}_{3} \mathrm{O}_{4} \mathrm{P}_{2} \mathrm{Ru}_{2}^{+}: m / z$ $1215.1119\left([\mathrm{M}-\mathrm{Cl}]^{+}\right)$. Found: $m / z$ 1215.1104. Calcd for $\mathrm{C}_{60} \mathrm{H}_{60} \mathrm{Cl}_{4} \mathrm{NaO}_{4} \mathrm{P}_{2} \mathrm{Ru}_{2}{ }^{+}: \mathrm{m} / z 1273.0706\left([\mathrm{M}+\mathrm{Na}]^{+}\right)$. Found: $\mathrm{m} / z$ 1273.0695. UV-vis: $\lambda_{\max }=250$ and $370 \mathrm{~nm}$.

Compound $2 b$. According to the general procedure, $\left[\mathrm{Ru}\left(\eta^{6}-p\right.\right.$ cymene $) \mathrm{Cl}_{2} \mathrm{Cl}_{2}$ (0.49 g, $0.808 \mathrm{mmol}, 1$ equiv), 2a (0.55 g, 0.808 mmol, 1 equiv), and $\mathrm{CH}_{2} \mathrm{Cl}_{2}(12 \mathrm{~mL})$ were stirred for $42 \mathrm{~h}$ at $\mathrm{RT}$. The product was isolated as a dark-red solid. Yield: $1.03 \mathrm{~g}, 0.791 \mathrm{mmol}$, 98\%. Elem anal. Calcd for $\mathrm{C}_{62} \mathrm{H}_{64} \mathrm{Cl}_{4} \mathrm{O}_{5} \mathrm{P}_{2} \mathrm{Ru}_{2}$ : C, 57.50; $\mathrm{H}, 4.98$. Found: C, 57.76; $\mathrm{H}, 5.12 .{ }^{1} \mathrm{H}$ NMR $\left(\mathrm{CDCl}_{3}\right): \delta_{\mathrm{H}} 7.86-7.96(8 \mathrm{H}, \mathrm{m}$, $4 \mathrm{O}(\mathrm{C}=\mathrm{O})(\mathrm{Ar}) \mathrm{CCHCHCP}, 4 \mathrm{O}(\mathrm{C}=\mathrm{O})(\mathrm{Ar}) \mathrm{CCHCHCP}), \quad 7.78-$ $7.83(8 \mathrm{H}, \mathrm{m}, 8 \mathrm{P}(A r) \mathrm{CCHCHCH}), 7.36-7.44(12 \mathrm{H}, \mathrm{m}, 8 \mathrm{P}(A r)-$ $\mathrm{CCHCHCH}, 4 \mathrm{P}(A r) \mathrm{CCHCHCH}), 5.21\left(4 \mathrm{H}, \mathrm{d}, 4 \mathrm{CH}_{3}(A r)-\right.$ $\left.\mathrm{CCHCHC},{ }^{3} J_{\mathrm{H}, \mathrm{H}}=6.2 \mathrm{~Hz}\right), 4.98\left(4 \mathrm{H}, \mathrm{d}, 4 \mathrm{CH}_{3}(\mathrm{Ar}) \mathrm{CCHCHC},{ }^{3} J_{\mathrm{H}, \mathrm{H}}\right.$ $=6.2 \mathrm{~Hz}), 4.45-4.42\left(4 \mathrm{H}, \mathrm{m}, 2 \mathrm{Ar}(\mathrm{C}=\mathrm{O}) \mathrm{OCH}_{2} \mathrm{CH}_{2} \mathrm{O}\right), 3.83-3.81$ $\left(4 \mathrm{H}, \quad \mathrm{m}, 2 \mathrm{Ar}(\mathrm{C}=\mathrm{O}) \mathrm{OCH}_{2} \mathrm{CH}_{2} \mathrm{O}\right), 2.85(2 \mathrm{H}$, sept, $2(\mathrm{Ar})-$ $\left.\mathrm{CCHCHCCH}\left(\mathrm{CH}_{3}\right)_{2},{ }^{3} \mathrm{~J}_{\mathrm{H}, \mathrm{H}}=6.9 \mathrm{~Hz}\right), 1.85\left(6 \mathrm{H}, \mathrm{s}, 2 \mathrm{CH}_{3}(\mathrm{Ar})\right.$ $\mathrm{CCHCHC}), 1.09\left(12 \mathrm{H}, \mathrm{d}, 2(\mathrm{Ar}) \mathrm{CCHCHCCH}\left(\mathrm{CH}_{3}\right)_{2},{ }^{3} J_{\mathrm{H}, \mathrm{H}}=6.9\right.$ $\mathrm{Hz}) .{ }^{31} \mathrm{P}$ NMR $\left(\mathrm{CDCl}_{3}\right): \delta_{\mathrm{P}} 24.95(2 \mathrm{P}) .{ }^{13} \mathrm{C} \mathrm{NMR}\left(\mathrm{CDCl}_{3}\right): \delta_{\mathrm{C}} 166.1$ 
$(2 \mathrm{C}, 2 \mathrm{O}(\mathrm{C}=\mathrm{O})(\mathrm{Ar}) \mathrm{CCHCHCP}), 139.5(2 \mathrm{C}, \mathrm{d}, 2 \mathrm{O}(\mathrm{C}=\mathrm{O})(A r)-$ $\left.\mathrm{CCHCHCP},{ }^{1} J_{\mathrm{C}, \mathrm{P}}=43 \mathrm{~Hz}\right), 134.5(4 \mathrm{C}, \mathrm{d}, 4 \mathrm{O}(\mathrm{C}=\mathrm{O})(A r)-$ $\left.\mathrm{CCHCHCP},{ }^{2} J_{\mathrm{C}, \mathrm{P}}=9 \mathrm{~Hz}\right), 134.4\left(8 \mathrm{C}, \mathrm{d}, 8 \mathrm{P}(\mathrm{Ar}) \mathrm{CCHCHCH},{ }^{2} J_{\mathrm{C}, \mathrm{P}}\right.$ $=9 \mathrm{~Hz}), 133.4\left(4 \mathrm{C}, \mathrm{d}, 4 \mathrm{P}(\mathrm{Ar}) \mathrm{CCHCHCH},{ }^{1} J_{\mathrm{C}, \mathrm{P}}=45 \mathrm{~Hz}\right), 131.3(2 \mathrm{C}$, $\left.\mathrm{d}, \quad 2 \mathrm{O}(\mathrm{C}=\mathrm{O})(A r) C \mathrm{CHCHCP},{ }^{4} J_{\mathrm{C}, \mathrm{P}}=2 \mathrm{~Hz}\right), 130.7(4 \mathrm{C}, \mathrm{d}$, $\left.4 \mathrm{P}(\mathrm{Ar}) \mathrm{CCHCHCH},{ }^{4} J_{\mathrm{C}, \mathrm{P}}=2 \mathrm{~Hz}\right), 128.8(4 \mathrm{C}, \mathrm{d}, 4 \mathrm{O}(\mathrm{C}=\mathrm{O})(\mathrm{Ar})-$ $\left.\mathrm{CCHCHCP},{ }^{3} J_{\mathrm{C}, \mathrm{P}}=10 \mathrm{~Hz}\right), 128.3\left(8 \mathrm{C}, \mathrm{d}, 8 \mathrm{P}(\mathrm{Ar}) \mathrm{CCHCHCH},{ }^{3} J_{\mathrm{C}, \mathrm{P}}=\right.$ $10 \mathrm{~Hz}), 111.59,111.56\left(2 \mathrm{C}, 2 \mathrm{CH}_{3}(\mathrm{Ar}) \mathrm{CCHCHC}\right), 96.4(2 \mathrm{C}$, $\left.2 \mathrm{CH}_{3}(\mathrm{Ar}) \mathrm{CCHCHC}\right), 89.15,89.12\left(4 \mathrm{C}, 4 \mathrm{CH}_{3}(\mathrm{Ar}) \mathrm{CCHCHC}\right)$, $87.49,87.43\left(4 \mathrm{C}, 4 \mathrm{CH}_{3}(\mathrm{Ar}) \mathrm{CCHCHC}\right), 69.4(2 \mathrm{C}, 2(\mathrm{Ar})(\mathrm{C}=$ O) $\left.\mathrm{OCH}_{2} \mathrm{CH}_{2} \mathrm{O}\right), 64.5\left(2 \mathrm{C}, 2(\mathrm{Ar})(\mathrm{C}=\mathrm{O}) \mathrm{OCH}_{2} \mathrm{CH}_{2} \mathrm{O}\right), 30.4(2 \mathrm{C}$, $\left.2(\mathrm{Ar}) \mathrm{CHCHCCH}\left(\mathrm{CH}_{3}\right)_{2}\right), 22.0\left(4 \mathrm{C}, 2(\mathrm{Ar}) \mathrm{CHCHCCH}\left(\mathrm{CH}_{3}\right)_{2}\right)$, $17.9\left(2 \mathrm{C}, 2 \mathrm{CH}_{3}(\mathrm{Ar}) \mathrm{CCHCH}\right)$. ESI-MS $(+)$. Calcd for $\mathrm{C}_{62} \mathrm{H}_{64} \mathrm{Cl}_{2} \mathrm{O}_{5} \mathrm{P}_{2} \mathrm{Ru}_{2}{ }^{+}: \mathrm{m} / z 1224.1693\left([\mathrm{M}-2 \mathrm{Cl}]^{+}\right)$. Found: $\mathrm{m} / z$ 1224.1804. Calcd for $\mathrm{C}_{62} \mathrm{H}_{64} \mathrm{Cl}_{3} \mathrm{O}_{5} \mathrm{P}_{2} \mathrm{Ru}_{2}{ }^{+}: \mathrm{m} / z$ 1259.1382 ([M $\mathrm{Cl}]^{+}$). Found: $m / z$ 1259.1396. Calcd for $\mathrm{C}_{62} \mathrm{H}_{64} \mathrm{Cl}_{4} \mathrm{NaO}_{5} \mathrm{P}_{2} \mathrm{Ru}_{2}{ }^{+}: m / z$ $1317.0962\left([\mathrm{M}+\mathrm{Na}]^{+}\right)$. Found: $m / z$ 1317.0995. UV-vis: $\lambda_{\max }=250$ and $370 \mathrm{~nm}$.

Compound $3 b$. According to the general procedure, $\left[\mathrm{Ru}\left(\eta^{6}-p\right.\right.$ cymene $\mathrm{Cl}_{2} \mathrm{Cl}_{2}$ (0.15 g, $0.251 \mathrm{mmol}, 1$ equiv), 3a (0.18 g, 0.251 mmol, 1 equiv), and $\mathrm{CH}_{2} \mathrm{Cl}_{2}(12 \mathrm{~mL})$ were stirred for $42 \mathrm{~h}$ at RT. The product was isolated as a dark-red solid. Yield: $0.33 \mathrm{~g}, 0.246 \mathrm{mmol}$, 98\%. Elem anal. Calcd for $\mathrm{C}_{64} \mathrm{H}_{66} \mathrm{Cl}_{4} \mathrm{O}_{6} \mathrm{P}_{2} \mathrm{Ru}_{2} \cdot \mathrm{CH}_{2} \mathrm{Cl}_{2}$ : C, 54.82; $\mathrm{H}$, 4.95. Found: $\mathrm{C}, 54.75, \mathrm{H}, 4.95 . \mathrm{CH}_{2} \mathrm{Cl}_{2}$ originates from the reaction solvent. ${ }^{1} \mathrm{H}$ NMR $\left(\mathrm{CDCl}_{3}\right): \delta_{\mathrm{H}} 7.86-7.94(8 \mathrm{H}, \mathrm{m}, \quad 4 \mathrm{O}(\mathrm{C}=$ $\mathrm{O})(\mathrm{Ar}) \mathrm{CCHCHCP}, 4 \mathrm{O}(\mathrm{C}=\mathrm{O})(\mathrm{Ar}) \mathrm{CCHCHCP}), 7.76-7.82(8 \mathrm{H}$, $\mathrm{m}, 8 \mathrm{P}(A r) \mathrm{CCHCHCH})$, 7.34-7.46 $(12 \mathrm{H}, \mathrm{m}, 8 \mathrm{P}(A r) \mathrm{CCHCHCH}$, $4 \mathrm{P}(\mathrm{Ar}) \mathrm{CCHCHCH}), 5.19\left(4 \mathrm{H}, \mathrm{d}, 4 \mathrm{CH}_{3}(\mathrm{Ar}) \mathrm{CCHCHC},{ }^{3} \mathrm{~J}_{\mathrm{H}, \mathrm{H}}=6.1\right.$ $\mathrm{Hz}), 4.96\left(4 \mathrm{H}, \mathrm{d}, 4 \mathrm{CH}_{3}(\mathrm{Ar}) \mathrm{CCHCHC},{ }^{3} J_{\mathrm{H}, \mathrm{H}}=6.1 \mathrm{~Hz}\right), 4.40-4.38$ $\left(4 \mathrm{H}, \mathrm{m}, 2 \mathrm{Ar}(\mathrm{C}=\mathrm{O}) \mathrm{OCH}_{2} \mathrm{CH}_{2} \mathrm{O}\right), 3.77-3.74(4 \mathrm{H}, \mathrm{m}, 2 \mathrm{Ar}(\mathrm{C}=$ $\left.\mathrm{O}) \mathrm{OCH}_{2} \mathrm{CH}_{2} \mathrm{O}\right), 3.65\left(4 \mathrm{H}, \mathrm{s}, 2 \mathrm{Ar}(\mathrm{C}=\mathrm{O}) \mathrm{O}\left(\mathrm{CH}_{2}\right)_{2} \mathrm{OCH}_{2}\right), 2.83(2 \mathrm{H}$, sept, $\left.2(\mathrm{Ar}) \mathrm{CCHCHCCH}\left(\mathrm{CH}_{3}\right)_{2},{ }^{3} J_{\mathrm{H}, \mathrm{H}}=6.9 \mathrm{~Hz}\right), 1.83(6 \mathrm{H}, \mathrm{s}$, $\left.2 \mathrm{CH}_{3}(\mathrm{Ar}) \mathrm{CCHCHC}\right), 1.08\left(12 \mathrm{H}, \mathrm{d}, 2(\mathrm{Ar}) \mathrm{CCHCHCCH}\left(\mathrm{CH}_{3}\right)_{2}\right.$, $\left.{ }^{3} J_{\mathrm{H}, \mathrm{H}}=6.9 \mathrm{~Hz}\right) .{ }^{31} \mathrm{P} \mathrm{NMR}\left(\mathrm{CDCl}_{3}\right): \delta_{\mathrm{P}} 24.94(2 \mathrm{P}) .{ }^{13} \mathrm{C} \mathrm{NMR}$ $\left(\mathrm{CDCl}_{3}\right): \delta_{\mathrm{C}} 166.0(2 \mathrm{C}, 2 \mathrm{O}(\mathrm{C}=\mathrm{O})-(\mathrm{Ar}) \mathrm{CCHCHCP}), 139.4(2 \mathrm{C}, \mathrm{d}$, $\left.2 \mathrm{O}(\mathrm{C}=\mathrm{O})(A r) \mathrm{CCHCHCP},{ }^{1} \mathrm{~J}_{\mathrm{C}, \mathrm{P}}=44 \mathrm{~Hz}\right), 134.4(4 \mathrm{C}, \mathrm{d}, 4 \mathrm{O}(\mathrm{C}=$ O) $\left.(A r) \mathrm{CCHCHCP},{ }^{2} J_{\mathrm{C}, \mathrm{P}}=8 \mathrm{~Hz}\right), 134.3(8 \mathrm{C}, \mathrm{d}, 8 \mathrm{P}(\mathrm{Ar}) \mathrm{CCHCHCH}$, $\left.{ }^{2} J_{\mathrm{C}, \mathrm{P}}=9 \mathrm{~Hz}\right), 133.4\left(4 \mathrm{C}, \mathrm{d}, 4 \mathrm{P}(\mathrm{Ar}) \mathrm{CCHCHCH},{ }^{1} J_{\mathrm{C}, \mathrm{P}}=45 \mathrm{~Hz}\right), 131.4$ $(2 \mathrm{C}, \mathrm{m}, 2 \mathrm{O}(\mathrm{C}=\mathrm{O})(A r) C \mathrm{CHCHCP}), 130.7$ (4C, m, 4P(Ar)$\mathrm{CCHCHCH}), 128.8\left(4 \mathrm{C}, \mathrm{d}, 4 \mathrm{O}(\mathrm{C}=\mathrm{O})(\mathrm{Ar}) \mathrm{CCHCHCP},{ }^{3} J_{\mathrm{C}, \mathrm{P}}=10\right.$ $\mathrm{Hz}), 128.3\left(8 \mathrm{C}, \mathrm{d}, 8 \mathrm{P}(\mathrm{Ar}) \mathrm{CCHCHCH},{ }^{3} J_{\mathrm{C}, \mathrm{P}}=10 \mathrm{~Hz}\right), 111.50,111.47$ (2C, $\left.2 \mathrm{CH}_{3}(\mathrm{Ar}) \mathrm{CCHCHC}\right), 96.4\left(2 \mathrm{C}, 2 \mathrm{CH}_{3}(\mathrm{Ar}) \mathrm{CCHCHC}\right), 89.09$, $89.07\left(4 \mathrm{C}, 4 \mathrm{CH}_{3}(\mathrm{Ar}) \mathrm{CCHCHC}\right), 87.43,87.38$ (4C, 4CH $\mathrm{CH}_{3}(\mathrm{Ar})$ $\mathrm{CCHCHC}), \quad 70.8\left(2 \mathrm{C}, 2(\mathrm{Ar})(\mathrm{C}=\mathrm{O}) \mathrm{O}\left(\mathrm{CH}_{2}\right)_{2} \mathrm{OCH}_{2} \mathrm{CH}_{2}\right), 69.3$ $\left(2 \mathrm{C}, \quad 2(\mathrm{Ar})(\mathrm{C}=\mathrm{O}) \mathrm{OCH}_{2} \mathrm{CH}_{2} \mathrm{O}\right), 64.4 \quad(2 \mathrm{C}, 2(A r)(\mathrm{C}=\mathrm{O})-$ $\left.\mathrm{OCH}_{2} \mathrm{CH}_{2} \mathrm{O}\right), 30.4\left(2 \mathrm{C}, 2(\mathrm{Ar}) \mathrm{CHCHCCH}\left(\mathrm{CH}_{3}\right)_{2}\right), 22.0$ (4C, $\left.2(\mathrm{Ar}) \mathrm{CHCHCCH}\left(\mathrm{CH}_{3}\right)_{2}\right), 17.9\left(2 \mathrm{C}, 2 \mathrm{CH}_{3}(\mathrm{Ar}) \mathrm{CCHCH}\right)$. ESIMS(+). Calcd for $\mathrm{C}_{64} \mathrm{H}_{68} \mathrm{Cl}_{3} \mathrm{O}_{6} \mathrm{P}_{2} \mathrm{Ru}_{2}{ }^{+}: m / z 1303.1644\left([\mathrm{M}-\mathrm{Cl}]^{+}\right)$. Found: $m / z$ 1303.1660. Calcd for $\mathrm{C}_{64} \mathrm{H}_{68} \mathrm{Cl}_{3} \mathrm{NaO}_{6} \mathrm{P}_{2} \mathrm{Ru}_{2}{ }^{+}: \mathrm{m} / z$ 1361.1224 $\left([\mathrm{M}+\mathrm{Na}]^{+}\right)$. Found: $m / z$ 1361.1219. UV-vis: $\lambda_{\max }=$ 250 and $370 \mathrm{~nm}$.

Compound $4 b$. According to the general procedure, $\left[\mathrm{Ru}\left(\eta^{6}-p\right.\right.$ cymene $) \mathrm{Cl}_{2} \mathrm{Cl}_{2}$ (0.13 g, $0.205 \mathrm{mmol}, 1$ equiv), 4a (0.16 g, 0.205 mmol, 1 equiv), and $\mathrm{CH}_{2} \mathrm{Cl}_{2}(12 \mathrm{~mL})$ were stirred for $42 \mathrm{~h}$ at $\mathrm{RT}$. The product was isolated as a red solid. Yield: $0.275 \mathrm{~g}, 0.199 \mathrm{mmol}, 97 \%$. Elem anal. Calcd for $\mathrm{C}_{66} \mathrm{H}_{72} \mathrm{Cl}_{4} \mathrm{O}_{7} \mathrm{P}_{2} \mathrm{Ru}_{2} \cdot{ }^{1}{ }_{2} \mathrm{CH}_{2} \mathrm{Cl}_{2}$ : C, 56.03; $\mathrm{H}, 5.06$. Found: $\mathrm{C}, 55.67 ; \mathrm{H}, 5.16 . \mathrm{CH}_{2} \mathrm{Cl}_{2}$ originates from the reaction solvent. ${ }^{1} \mathrm{H}$ NMR $\left(\mathrm{CDCl}_{3}\right): \delta_{\mathrm{H}} 7.85-7.95(8 \mathrm{H}, \mathrm{m}, 4 \mathrm{O}(\mathrm{C}=\mathrm{O})(\mathrm{Ar})-$ CCHCHCP, $4 \mathrm{O}(\mathrm{C}=\mathrm{O})(\mathrm{Ar}) \mathrm{CCHCHCP}), 7.76-7.81(8 \mathrm{H}, \mathrm{m}, 8 \mathrm{P}-$ $(A r) \mathrm{CCHCHCH}), 7.34-7.42(12 \mathrm{H}, \mathrm{m}, 8 \mathrm{P}(\mathrm{Ar}) \mathrm{CCHCHCH}, 4 \mathrm{P}(\mathrm{Ar})-$ $\mathrm{CCHCHCH}), 5.19\left(4 \mathrm{H}, \mathrm{d}, 4 \mathrm{CH}_{3}(\mathrm{Ar}) \mathrm{CCHCHC},{ }^{3} J_{\mathrm{H}, \mathrm{H}}=6.2 \mathrm{~Hz}\right), 4.96$ $\left(4 \mathrm{H}, \mathrm{d}, 4 \mathrm{CH}_{3}(\mathrm{Ar}) \mathrm{CCHCHC},{ }^{3} \mathrm{~J}_{\mathrm{H}, \mathrm{H}}=6.2 \mathrm{~Hz}\right), 4.41-4.38(4 \mathrm{H}, \mathrm{m}$, $\left.2 \mathrm{Ar}(\mathrm{C}=\mathrm{O}) \mathrm{OCH}_{2} \mathrm{CH}_{2} \mathrm{O}\right), 3.75-3.73(4 \mathrm{H}, \mathrm{m}, 2 \mathrm{Ar}(\mathrm{C}=\mathrm{O})-$ $\left.\mathrm{OCH}_{2} \mathrm{CH}_{2} \mathrm{O}\right), 3.64-3.60\left(8 \mathrm{H}, \mathrm{m}, 2 \mathrm{Ar}(\mathrm{C}=\mathrm{O}) \mathrm{O}\left(\mathrm{CH}_{2}\right)_{2} \mathrm{OCH}_{2} \mathrm{CH}_{2}\right.$, $\left.2 \mathrm{Ar}(\mathrm{C}=\mathrm{O}) \mathrm{O}\left(\mathrm{CH}_{2}\right)_{2} \mathrm{OCH}_{2} \mathrm{CH}_{2}\right), 2.83(2 \mathrm{H}$, sept, $2(\mathrm{Ar})$ $\left.\mathrm{CCHCHCCH}\left(\mathrm{CH}_{3}\right)_{2},{ }^{3} J_{\mathrm{H}, \mathrm{H}}=6.9 \mathrm{~Hz}\right), 1.83\left(6 \mathrm{H}, \mathrm{s}, 2 \mathrm{CH}_{3}(\mathrm{Ar})-\right.$ $\mathrm{CCHCHC}), 1.08\left(12 \mathrm{H}, \mathrm{d}, 2(\mathrm{Ar}) \mathrm{CCHCHCCH}\left(\mathrm{CH}_{3}\right)_{2},{ }^{3} \mathrm{~J}_{\mathrm{H}, \mathrm{H}}=6.9\right.$ $\mathrm{Hz}) .{ }^{31} \mathrm{P}$ NMR $\left(\mathrm{CDCl}_{3}\right): \delta_{\mathrm{P}} 24.96(2 \mathrm{P}) .{ }^{13} \mathrm{C} \mathrm{NMR}\left(\mathrm{CDCl}_{3}\right): \delta_{\mathrm{C}} 166.1$ $(2 \mathrm{C}, 2 \mathrm{O}(\mathrm{C}=\mathrm{O})(\mathrm{Ar}) \mathrm{CCHCHCP}), 139.4(2 \mathrm{C}, \mathrm{d}, 2 \mathrm{O}(\mathrm{C}=\mathrm{O})(A r)-$ $\left.\mathrm{CCHCHCP},{ }^{1} J_{\mathrm{C}, \mathrm{P}}=44 \mathrm{~Hz}\right), 134.5(4 \mathrm{C}, \mathrm{d}, 4 \mathrm{O}(\mathrm{C}=\mathrm{O})(\mathrm{Ar})$ -
$\left.\mathrm{CCHCHCP},{ }^{2} J_{\mathrm{C}, \mathrm{P}}=8 \mathrm{~Hz}\right), 134.4\left(8 \mathrm{C}, \mathrm{d}, 8 \mathrm{P}(\mathrm{Ar}) \mathrm{CCHCHCH},{ }^{2} J_{\mathrm{C}, \mathrm{P}}\right.$ $=9 \mathrm{~Hz}), 133.4\left(4 \mathrm{C}, \mathrm{d}, 4 \mathrm{P}(\mathrm{Ar}) \mathrm{CCHCHCH},{ }^{1} J_{\mathrm{C}, \mathrm{P}}=45 \mathrm{~Hz}\right), 131.4(2 \mathrm{C}$, $\left.\mathrm{d}, 2 \mathrm{O}(\mathrm{C}=\mathrm{O})(\mathrm{Ar}) \mathrm{CCHCHCP},{ }^{4} J_{\mathrm{C}, \mathrm{P}}=2 \mathrm{~Hz}\right), 130.7(4 \mathrm{C}, \mathrm{d}$, $\left.4 \mathrm{P}(\mathrm{Ar}) \mathrm{CCHCHCH},{ }^{4} \mathrm{~J}_{\mathrm{C}, \mathrm{P}}=2 \mathrm{~Hz}\right), 128.8(4 \mathrm{C}, \mathrm{d}, 4 \mathrm{O}(\mathrm{C}=\mathrm{O})(\mathrm{Ar})-$ CCHCHCP, $\left.{ }^{3} J_{\mathrm{C}, \mathrm{P}}=10 \mathrm{~Hz}\right), 128.3\left(8 \mathrm{C}, \mathrm{d}, 8 \mathrm{P}(\mathrm{Ar}) \mathrm{CCHCHCH},{ }^{3} J_{\mathrm{C}, \mathrm{P}}=\right.$ $10 \mathrm{~Hz}), 111.54,111.50\left(2 \mathrm{C}, 2 \mathrm{CH}_{3}(\mathrm{Ar}) \mathrm{CCHCHC}\right), 96.4(2 \mathrm{C}$, $\left.2 \mathrm{CH}_{3}(\mathrm{Ar}) \mathrm{CCHCHC}\right), 89.11,89.09$ (4C, 4CH $\left.(\mathrm{Ar}) \mathrm{CCHCHC}\right)$, 87.46, $87.40\left(4 \mathrm{C}, 4 \mathrm{CH}_{3}(\mathrm{Ar}) \mathrm{CCHCHC}\right), 70.8,70.4(4 \mathrm{C}, 2(\mathrm{Ar})(\mathrm{C}=$ $\left.\mathrm{O}) \mathrm{O}\left(\mathrm{CH}_{2}\right)_{2} \mathrm{OCH}_{2}, 2(\mathrm{Ar})(\mathrm{C}=\mathrm{O}) \mathrm{O}\left(\mathrm{CH}_{2}\right)_{2} \mathrm{OCH}_{2} \mathrm{CH}_{2}\right), 69.2(2 \mathrm{C}$, $\left.2(\mathrm{Ar})(\mathrm{C}=\mathrm{O}) \mathrm{OCH}_{2} \mathrm{CH}_{2} \mathrm{O}\right), 64.5\left(2 \mathrm{C}, 2(\mathrm{Ar})(\mathrm{C}=\mathrm{O}) \mathrm{OCH}_{2} \mathrm{CH}_{2} \mathrm{O}\right)$, $30.4\left(2 \mathrm{C}, 2(\mathrm{Ar}) \mathrm{CHCHCCH}\left(\mathrm{CH}_{3}\right)_{2}\right), 22.0$ (4C, $2(\mathrm{Ar}) \mathrm{CHCHCCH}-$ $\left.\left(\mathrm{CH}_{3}\right)_{2}\right), 17.9\left(2 \mathrm{C}, 2 \mathrm{CH}_{3}(\mathrm{Ar}) \mathrm{CCHCH}\right)$. ESI-MS $(+)$. Calcd for $\mathrm{C}_{66} \mathrm{H}_{72} \mathrm{Cl}_{2} \mathrm{O}_{7} \mathrm{P}_{2} \mathrm{Ru}_{2}{ }^{2+}: 1312.2206\left([\mathrm{M}-2 \mathrm{Cl}]^{+}\right)$. Found: $\mathrm{m} / z$ 1312.2328. Calcd for $\mathrm{C}_{66} \mathrm{H}_{72} \mathrm{Cl}_{3} \mathrm{O}_{7} \mathrm{P}_{2} \mathrm{Ru}_{2}^{+}: 1347.1900\left([\mathrm{M}-\mathrm{Cl}]^{+}\right)$. Found: $m / z$ 1347.1923. Calcd for $\mathrm{C}_{66} \mathrm{H}_{72} \mathrm{Cl}_{4} \mathrm{NaO}_{7} \mathrm{P}_{2} \mathrm{Ru}_{2}{ }^{+}$: 1405.1487 $\left([\mathrm{M}+\mathrm{Na}]^{+}\right)$. Found: $m / z$ 1405.1474. UV-vis: $\lambda_{\max }=250$ and 370 $\mathrm{nm}$.

Compound 5b. According to the general procedure, $\left[\mathrm{Ru}\left(\eta^{6}-p\right.\right.$ cymene $\mathrm{Cl}_{2} \mathrm{Cl}_{2}$ (0.16 g, $0.269 \mathrm{mmol}, 1$ equiv), 5a (0.22 g, 0.269 mmol, 1 equiv), and $\mathrm{CH}_{2} \mathrm{Cl}_{2}(12 \mathrm{~mL})$ were stirred for $42 \mathrm{~h}$ at $\mathrm{RT}$. The product was isolated as a red solid. Yield: $0.38 \mathrm{~g}, 0.263 \mathrm{mmol}, 98 \%$. Elem anal. Calcd for $\mathrm{C}_{68} \mathrm{H}_{76} \mathrm{Cl}_{4} \mathrm{O}_{8} \mathrm{P}_{2} \mathrm{Ru}_{2} \cdot{ }^{1}{ }_{2} \mathrm{CH}_{2} \mathrm{Cl}_{2}$ : C, 55.98; $\mathrm{H}, 5.28$. Found: $\mathrm{C}, 55.86 ; \mathrm{H}, 5.34 . \mathrm{CH}_{2} \mathrm{Cl}_{2}$ originates from the reaction solvent. ${ }^{1} \mathrm{H}$ NMR $\left(\mathrm{CDCl}_{3}\right): \delta_{\mathrm{H}} 7.88-7.97(8 \mathrm{H}, \mathrm{m}, \quad 4 \mathrm{O}(\mathrm{C}=\mathrm{O})(\mathrm{Ar})-$ $\mathrm{CCHCHCP,} 4 \mathrm{O}(\mathrm{C}=\mathrm{O})(\mathrm{Ar}) \mathrm{CCHCHCP}), 7.77-7.81(8 \mathrm{H}, \mathrm{m}, 8 \mathrm{P}-$ $(A r) \mathrm{CCHCHCH}), 7.35-7.42(12 \mathrm{H}, \mathrm{m}, 8 \mathrm{P}(A r) \mathrm{CCHCHCH}, 4 \mathrm{P}(A r)-$ $\mathrm{CCHCHCH}), 5.20\left(4 \mathrm{H}, \mathrm{d}, 4 \mathrm{CH}_{3}(\mathrm{Ar}) \mathrm{CCHCHC},{ }^{3} J_{\mathrm{H}, \mathrm{H}}=6.1 \mathrm{~Hz}\right), 4.96$ $\left(4 \mathrm{H}, \mathrm{d}, 4 \mathrm{CH}_{3}(\mathrm{Ar}) \mathrm{CCHCHC},{ }^{3} \mathrm{~J}_{\mathrm{H}, \mathrm{H}}=6.1 \mathrm{~Hz}\right), 4.42-4.39(4 \mathrm{H}, \mathrm{m}$, $\left.2 \mathrm{Ar}(\mathrm{C}=\mathrm{O}) \mathrm{OCH}_{2} \mathrm{CH}_{2} \mathrm{O}\right), 3.77-3.74(4 \mathrm{H}, \mathrm{m}, 2 \mathrm{Ar}(\mathrm{C}=\mathrm{O})-$ $\left.\mathrm{OCH}_{2} \mathrm{CH}_{2} \mathrm{O}\right), 3.65-3.56\left(12 \mathrm{H}, \mathrm{m}, 2 \mathrm{Ar}(\mathrm{C}=\mathrm{O}) \mathrm{O}\left(\mathrm{CH}_{2}\right)_{2} \mathrm{OCH}_{2} \mathrm{CH}_{2}\right.$, $2 \mathrm{Ar}(\mathrm{C}=\mathrm{O}) \mathrm{O}\left(\mathrm{CH}_{2}\right)_{2} \mathrm{OCH}_{2} \mathrm{CH}_{2}, \quad 2 \mathrm{Ar}(\mathrm{C}=\mathrm{O}) \mathrm{O}\left(\mathrm{CH}_{2}\right)_{2} \mathrm{O}-$ $\left.\left(\mathrm{CH}_{2}\right)_{2} \mathrm{OCH}_{2}\right), 2.83\left(2 \mathrm{H}\right.$, sept, $2(\mathrm{Ar}) \mathrm{CCHCHCCH}\left(\mathrm{CH}_{3}\right)_{2},{ }^{3} J_{\mathrm{H}, \mathrm{H}}=$ $6.9 \mathrm{~Hz}), 1.84\left(6 \mathrm{H}, \mathrm{s}, 2 \mathrm{CH}_{3}(\mathrm{Ar}) \mathrm{CCHCHC}\right), 1.09(12 \mathrm{H}, \mathrm{d}$, $\left.2(\mathrm{Ar}) \mathrm{CCHCHCCH}\left(\mathrm{CH}_{3}\right)_{2},{ }^{3} \mathrm{~J}_{\mathrm{H}, \mathrm{H}}=6.9 \mathrm{~Hz}\right) \cdot{ }^{31} \mathrm{P} \mathrm{NMR}\left(\mathrm{CDCl}_{3}\right): \delta_{\mathrm{P}}$ $24.96(2 \mathrm{P}) .{ }^{13} \mathrm{C}$ NMR $\left(\mathrm{CDCl}_{3}\right): \delta_{\mathrm{C}} 166.1(2 \mathrm{C}, 2 \mathrm{O}(\mathrm{C}=\mathrm{O})(\mathrm{Ar})-$ $\mathrm{CCHCHCP}), 139.4\left(2 \mathrm{C}, \mathrm{d}, 2 \mathrm{O}(\mathrm{C}=\mathrm{O})(\mathrm{Ar}) \mathrm{CCHCHCP},{ }_{\mathrm{C}, \mathrm{P}}=44\right.$ $\mathrm{Hz}), 134.5\left(4 \mathrm{C}, \mathrm{d}, 4 \mathrm{O}(\mathrm{C}=\mathrm{O})(\mathrm{Ar}) \mathrm{CCHCHCP},{ }^{2} J_{\mathrm{C}, \mathrm{P}}=7 \mathrm{~Hz}\right), 134.4$ $\left(8 \mathrm{C}, \mathrm{d}, 8 \mathrm{P}(A r) \mathrm{CCHCHCH},{ }^{2} \mathrm{~J}_{\mathrm{C}, \mathrm{P}}=9 \mathrm{~Hz}\right), 133.4(4 \mathrm{C}, \mathrm{d}, 4 \mathrm{P}(A r)$ $\left.\mathrm{CCHCHCH},{ }^{1} J_{\mathrm{C}, \mathrm{P}}=45 \mathrm{~Hz}\right), 131.4(2 \mathrm{C}, \mathrm{d}, 2 \mathrm{O}(\mathrm{C}=\mathrm{O})(\mathrm{Ar})$ CCHCHCP, $\left.{ }^{4} J_{\mathrm{C}, \mathrm{P}}=2 \mathrm{~Hz}\right), 130.7\left(4 \mathrm{C}, \mathrm{d}, 4 \mathrm{P}(\mathrm{Ar}) \mathrm{CCHCHCH},{ }^{4} J_{\mathrm{C}, \mathrm{P}}\right.$ $=2 \mathrm{~Hz}), 128.8\left(4 \mathrm{C}, \mathrm{d}, 4 \mathrm{O}(\mathrm{C}=\mathrm{O})(\mathrm{Ar}) \mathrm{CCHCHCP},{ }^{3} \mathrm{~J}_{\mathrm{C}, \mathrm{P}}=10 \mathrm{~Hz}\right)$, $128.3\left(8 \mathrm{C}, \mathrm{d}, 8 \mathrm{P}(\mathrm{Ar}) \mathrm{CCHCHCH},{ }^{3} \mathrm{~J}_{\mathrm{C}, \mathrm{P}}=10 \mathrm{~Hz}\right), 111.56,111.53(2 \mathrm{C}$, $\left.2 \mathrm{CH}_{3}(\mathrm{Ar}) \mathrm{CCHCHC}\right), 96.4\left(2 \mathrm{C}, 2 \mathrm{CH}_{3}(\mathrm{Ar}) \mathrm{CCHCHC}\right), 89.09,89.11$ (4C, $\left.4 \mathrm{CH}_{3}(\mathrm{Ar}) \mathrm{CCHCHC}\right), 87.41,87.46\left(4 \mathrm{C}, 4 \mathrm{CH}_{3}(\mathrm{Ar}) \mathrm{CCHCHC}\right)$, $70.8,70.7\left(6 \mathrm{C}, 2(A r)(\mathrm{C}=\mathrm{O}) \mathrm{O}\left(\mathrm{CH}_{2}\right)_{2} \mathrm{OCH}_{2}, 2(\mathrm{Ar})(\mathrm{C}=\mathrm{O}) \mathrm{O}-\right.$ $\left.\left(\mathrm{CH}_{2}\right)_{2} \mathrm{OCH}_{2} \mathrm{CH}_{2}, 2(\mathrm{Ar})(\mathrm{C}=\mathrm{O}) \mathrm{O}\left(\mathrm{CH}_{2}\right)_{2} \mathrm{O}\left(\mathrm{CH}_{2}\right)_{2} \mathrm{OCH}_{2}\right), 69.2$ $\left(2 \mathrm{C}, 2(\mathrm{Ar})(\mathrm{C}=\mathrm{O}) \mathrm{OCH}_{2} \mathrm{CH}_{2} \mathrm{O}\right), 64.5(2 \mathrm{C}, 2(\mathrm{Ar})(\mathrm{C}=\mathrm{O})-$ $\left.\mathrm{OCH}_{2} \mathrm{CH}_{2} \mathrm{O}\right), 30.4\left(2 \mathrm{C}, 2(\mathrm{Ar}) \mathrm{CHCHCCH}\left(\mathrm{CH}_{3}\right)_{2}\right), 22.0$ (4C, $\left.2(\mathrm{Ar}) \mathrm{CHCHCCH}\left(\mathrm{CH}_{3}\right)_{2}\right), 17.9\left(2 \mathrm{C}, 2 \mathrm{CH}_{3}(\mathrm{Ar}) \mathrm{CCHCH}\right)$. ESI$\mathrm{MS}(+)$. Calcd for $\mathrm{C}_{68} \mathrm{H}_{76} \mathrm{Cl}_{2} \mathrm{O}_{8} \mathrm{P}_{2} \mathrm{Ru}_{2}{ }^{2+}: \mathrm{m} / z$ 1356.2583 ([M $\left.2 \mathrm{Cl}]^{+}\right)$. Found: $m / z$ 1356.2609. Calcd for $\mathrm{C}_{68} \mathrm{H}_{76} \mathrm{Cl}_{3} \mathrm{O}_{8} \mathrm{P}_{2} \mathrm{Ru}_{2}{ }^{+}: \mathrm{m} / z$ $1391.2168\left([\mathrm{M}-\mathrm{Cl}]^{+}\right)$. Found: $m / z$ 1391.2207. Calcd for $\mathrm{C}_{68} \mathrm{H}_{76} \mathrm{Cl}_{4} \mathrm{NaO}_{8} \mathrm{P}_{2} \mathrm{Ru}_{2}^{+}: m / z 1449.1749\left([\mathrm{M}+\mathrm{Na}]^{+}\right)$. Found: $m / z$ 1449.1803. UV-vis: $\lambda_{\max }=250$ and $370 \mathrm{~nm}$.

Compound $6 b$. According to the general procedure, $\left[\mathrm{Ru}\left(\eta^{6}-p\right.\right.$ cymene $) \mathrm{Cl}_{2} \mathrm{Cl}_{2}$ (0.16 g, $0.261 \mathrm{mmol}, 1$ equiv), 6a (0.22 g, 0.261 mmol, 1 equiv), and $\mathrm{CH}_{2} \mathrm{Cl}_{2}(12 \mathrm{~mL})$ were stirred for $42 \mathrm{~h}$ at $\mathrm{RT}$. The product was isolated as a red solid. Yield: $0.38 \mathrm{~g}, 0.256 \mathrm{mmol}, 98 \%$. Elem anal. Calcd for $\mathrm{C}_{70} \mathrm{H}_{80} \mathrm{Cl}_{4} \mathrm{O}_{9} \mathrm{P}_{2} \mathrm{Ru}_{2}: \mathrm{C}, 57.14 ; \mathrm{H}, 5.48$. Found: $\mathrm{C}$, 57.05, H, 5.64. ${ }^{1} \mathrm{H}$ NMR $\left(\mathrm{CDCl}_{3}\right): \delta_{\mathrm{H}} 7.88-7.97(8 \mathrm{H}, \mathrm{m}, 4 \mathrm{O}(\mathrm{C}=$ O) $(\mathrm{Ar}) \mathrm{CCHCHCP}, 4 \mathrm{O}(\mathrm{C}=\mathrm{O})(\mathrm{Ar}) \mathrm{CCHCHCP}), 7.78-7.83(8 \mathrm{H}, \mathrm{m}$, $8 \mathrm{P}(A r) \mathrm{CCHCHCH}), 7.36-7.45(12 \mathrm{H}, \mathrm{m}, 8 \mathrm{P}(\mathrm{Ar}) \mathrm{CCHCHCH}, 4 \mathrm{P}-$ $(\mathrm{Ar}) \mathrm{CCHCHCH}), 5.21\left(4 \mathrm{H}, \mathrm{d}, 4 \mathrm{CH}_{3}(\mathrm{Ar}) \mathrm{CCHCHC},{ }^{3} J_{\mathrm{H}, \mathrm{H}}=6.2 \mathrm{~Hz}\right)$, $4.97\left(4 \mathrm{H}, \mathrm{d}, 4 \mathrm{CH}_{3}(\mathrm{Ar}) \mathrm{CCHCHC},{ }^{3} J_{\mathrm{H}, \mathrm{H}}=6.2 \mathrm{~Hz}\right), 4.44-4.41(4 \mathrm{H}, \mathrm{m}$, $\left.2 \mathrm{Ar}(\mathrm{C}=\mathrm{O}) \mathrm{OCH}_{2} \mathrm{CH}_{2} \mathrm{O}\right), \quad 3.79-3.77(4 \mathrm{H}, \mathrm{m}, \quad 2 \mathrm{Ar}(\mathrm{C}=\mathrm{O})-$ $\left.\mathrm{OCH}_{2} \mathrm{CH}_{2} \mathrm{O}\right), 3.67-3.61\left(16 \mathrm{H}, \mathrm{m}, 2 \mathrm{Ar}(\mathrm{C}=\mathrm{O}) \mathrm{O}\left(\mathrm{CH}_{2}\right)_{2} \mathrm{OCH}_{2} \mathrm{CH}_{2}\right.$, $2 \mathrm{Ar}(\mathrm{C}=\mathrm{O}) \mathrm{O}\left(\mathrm{CH}_{2}\right)_{2} \mathrm{OCH}_{2} \mathrm{CH}_{2}, 2 \mathrm{Ar}(\mathrm{C}=\mathrm{O}) \mathrm{O}\left(\mathrm{CH}_{2}\right)_{2} \mathrm{O}$ $\left.\left.\left(\mathrm{CH}_{2}\right)_{2} \mathrm{OCH}_{2}\right), 2 \mathrm{Ar}(\mathrm{C}=\mathrm{O}) \mathrm{O}\left(\mathrm{CH}_{2}\right)_{2} \mathrm{O}\left(\mathrm{CH}_{2}\right)_{2} \mathrm{OCH}_{2} \mathrm{CH}_{2}\right), 2.85$ $(2 \mathrm{H}$, sept, 2( $\left.\mathrm{Ar}) \mathrm{CCHCHCCH}\left(\mathrm{CH}_{3}\right)_{2},{ }^{3} J_{\mathrm{H}, \mathrm{H}}=6.9 \mathrm{~Hz}\right), 1.86(6 \mathrm{H}, \mathrm{s}$, $\left.2 \mathrm{CH}_{3}(\mathrm{Ar}) \mathrm{CCHCHC}\right), 1.10\left(12 \mathrm{H}, \mathrm{d}, 2(\mathrm{Ar}) \mathrm{CCHCHCCH}\left(\mathrm{CH}_{3}\right)_{2}\right.$, 
$\left.{ }^{3} J_{\mathrm{H}, \mathrm{H}}=6.9 \mathrm{~Hz}\right) .{ }^{31} \mathrm{P} \mathrm{NMR}\left(\mathrm{CDCl}_{3}\right): \delta_{\mathrm{P}} 24.96(2 \mathrm{P}) .{ }^{13} \mathrm{C} \mathrm{NMR}$ $\left(\mathrm{CDCl}_{3}\right): \delta_{\mathrm{C}} 166.1(2 \mathrm{C}, 2 \mathrm{O}(\mathrm{C}=\mathrm{O})(\mathrm{Ar}) \mathrm{CCHCHCP}), 139.4(2 \mathrm{C}, \mathrm{d}$, $\left.2 \mathrm{O}(\mathrm{C}=\mathrm{O})(\mathrm{Ar}) \mathrm{CCHCHCP},{ }^{1} \mathrm{~J}_{\mathrm{C}, \mathrm{P}}=44 \mathrm{~Hz}\right), 134.5(4 \mathrm{C}, \mathrm{d}, 4 \mathrm{O}(\mathrm{C}=$ O) $\left.(A r) \mathrm{CCHCHCP},{ }^{2} J_{\mathrm{C}, \mathrm{P}}=7 \mathrm{~Hz}\right), 134.4(8 \mathrm{C}, \mathrm{d}, 8 \mathrm{P}(\mathrm{Ar}) \mathrm{CCHCHCH}$ $\left.{ }^{2} J_{\mathrm{C}, \mathrm{P}}=9 \mathrm{~Hz}\right), 133.4\left(4 \mathrm{C}, \mathrm{d}, 4 \mathrm{P}(\mathrm{Ar}) \mathrm{CCHCHCH},{ }^{1} J_{\mathrm{C}, \mathrm{P}}=45 \mathrm{~Hz}\right), 131.4$ $\left(2 \mathrm{C}, \mathrm{d}, 2 \mathrm{O}(\mathrm{C}=\mathrm{O})(\mathrm{Ar}) \mathrm{CCHCHCP},{ }^{4} J_{\mathrm{C}, \mathrm{P}}=2 \mathrm{~Hz}\right), 130.7(4 \mathrm{C}, \mathrm{d}$, $\left.4 \mathrm{P}(\mathrm{Ar}) \mathrm{CCHCHCH},{ }^{4} J_{\mathrm{C}, \mathrm{P}}=2 \mathrm{~Hz}\right), 128.8(4 \mathrm{C}, \mathrm{d}, 4 \mathrm{O}(\mathrm{C}=\mathrm{O})(\mathrm{Ar})-$ $\left.\mathrm{CCHCHCP},{ }^{3} J_{\mathrm{C}, \mathrm{P}}=10 \mathrm{~Hz}\right), 128.3\left(8 \mathrm{C}, \mathrm{d}, 8 \mathrm{P}(\mathrm{Ar}) \mathrm{CCHCHCH},{ }^{3} J_{\mathrm{C}, \mathrm{P}}=\right.$ $10 \mathrm{~Hz}), 111.61,111.58\left(2 \mathrm{C}, 2 \mathrm{CH}_{3}(\mathrm{Ar}) \mathrm{CCHCHC}\right), 96.4(2 \mathrm{C}$, $\left.2 \mathrm{CH}_{3}(\mathrm{Ar}) \mathrm{CCHCHC}\right), 89.13,89.10\left(4 \mathrm{C}, 4 \mathrm{CH}_{3}(\mathrm{Ar}) \mathrm{CCHCHC}\right)$, $87.49,87.44\left(4 \mathrm{C}, 4 \mathrm{CH}_{3}(\mathrm{Ar}) \mathrm{CCHCHC}\right), 70.82,70.73,70.67$ (8C, $2(\mathrm{Ar})(\mathrm{C}=\mathrm{O}) \mathrm{O}\left(\mathrm{CH}_{2}\right)_{2} \mathrm{OCH}_{2}, 2(\mathrm{Ar})(\mathrm{C}=\mathrm{O}) \mathrm{O}\left(\mathrm{CH}_{2}\right)_{2} \mathrm{OCH}_{2} \mathrm{CH}_{2}, 2-$ $(\mathrm{Ar})(\mathrm{C}=\mathrm{O}) \mathrm{O}\left(\mathrm{CH}_{2}\right)_{2} \mathrm{O}\left(\mathrm{CH}_{2}\right)_{2} \mathrm{OCH}_{2}, 2(\mathrm{Ar})(\mathrm{C}=\mathrm{O}) \mathrm{O}\left(\mathrm{CH}_{2}\right)_{2} \mathrm{O}-$ $\left.\left(\mathrm{CH}_{2}\right)_{2} \mathrm{OCH}_{2} \mathrm{CH}_{2}\right), 69.2\left(2 \mathrm{C}, 2(\mathrm{Ar})(\mathrm{C}=\mathrm{O}) \mathrm{OCH}_{2} \mathrm{CH}_{2} \mathrm{O}\right), 64.5$ $\left(2 \mathrm{C}, 2(\mathrm{Ar})(\mathrm{C}=\mathrm{O}) \mathrm{OCH}_{2} \mathrm{CH}_{2} \mathrm{O}\right), 30.4(2 \mathrm{C}, 2(\mathrm{Ar}) \mathrm{CHCHCCH}-$ $\left.\left(\mathrm{CH}_{3}\right)_{2}\right), 22.0\left(4 \mathrm{C}, 2(\mathrm{Ar}) \mathrm{CHCHCCH}\left(\mathrm{CH}_{3}\right)_{2}\right), 17.9\left(2 \mathrm{C}, 2 \mathrm{CH}_{3}(\mathrm{Ar})-\right.$ $\mathrm{CCHCH})$. ESI-MS(+). Calcd for $\mathrm{C}_{70} \mathrm{H}_{80} \mathrm{Cl}_{2} \mathrm{O}_{9} \mathrm{P}_{2} \mathrm{Ru}_{2}{ }^{2+}: \mathrm{m} / z$ $1400.2742\left([\mathrm{M}-2 \mathrm{Cl}]^{+}\right)$. Found: $\mathrm{m} / z$ 1400.2695. Calcd for $\mathrm{C}_{70} \mathrm{H}_{80} \mathrm{Cl}_{3} \mathrm{O}_{9} \mathrm{P}_{2} \mathrm{Ru}_{2}^{+}: m / z$ 1435.2430 $\left([\mathrm{M}-\mathrm{Cl}]^{+}\right)$. Found: $\mathrm{m} / z$ 1435.2384. Calcd for $\mathrm{C}_{70} \mathrm{H}_{80} \mathrm{Cl}_{4} \mathrm{NaO}_{9} \mathrm{P}_{2} \mathrm{Ru}_{2}{ }^{+}: m / z 1493.2016([\mathrm{M}+$ $\mathrm{Na}]^{+}$). Found: $m / z$ 1493.2328. UV-vis: $\lambda_{\max }=250$ and $370 \mathrm{~nm}$.

Compound $7 b$. According to the general procedure, $\left[\mathrm{Ru}\left(\eta^{6}-p\right.\right.$ cymene) $\mathrm{Cl}_{2} \mathrm{Cl}_{2}$ (0.18 g, $0.299 \mathrm{mmol}, 1$ equiv), $7 \mathrm{a}$ (0.28 g, 0.299 mmol 1 equiv), and $\mathrm{CH}_{2} \mathrm{Cl}_{2}(12 \mathrm{~mL})$ were stirred for $42 \mathrm{~h}$ at RT. The product was isolated as a red solid. Yield: $0.45 \mathrm{~g}, 0.287 \mathrm{mmol}, 96 \%$. Elem anal. Calcd for $\mathrm{C}_{74} \mathrm{H}_{88} \mathrm{Cl}_{4} \mathrm{O}_{11} \mathrm{P}_{2} \mathrm{Ru}_{2}$ : C, 57.00; $\mathrm{H}, 5.69$. Found: $\mathrm{C}$, 56.95; $\mathrm{H}, 5.90 .{ }^{1} \mathrm{H}$ NMR $\left(\mathrm{CDCl}_{3}\right): \delta_{\mathrm{H}} 7.88-7.98(8 \mathrm{H}, \mathrm{m}, 4 \mathrm{O}(\mathrm{C}=$ $\mathrm{O})(\mathrm{Ar}) \mathrm{CCHCHCP}, 4 \mathrm{O}(\mathrm{C}=\mathrm{O})(\mathrm{Ar}) \mathrm{CCHCHCP}), 7.77-7.83(8 \mathrm{H}, \mathrm{m}$, $8 \mathrm{P}(\mathrm{Ar}) \mathrm{CCHCHCH}), 7.36-7.45(12 \mathrm{H}, \mathrm{m}, 8 \mathrm{P}(\mathrm{Ar}) \mathrm{CCHCHCH}, 4 \mathrm{P}-$ $(A r) \mathrm{CCHCHCH}), 5.21\left(4 \mathrm{H}, \mathrm{d}, 4 \mathrm{CH}_{3}(A r) \mathrm{CCHCHC},{ }^{3} J_{\mathrm{H}, \mathrm{H}}=6.2 \mathrm{~Hz}\right)$, $4.98\left(4 \mathrm{H}, \mathrm{d}, 4 \mathrm{CH}_{3}(\mathrm{Ar}) \mathrm{CCHCHC},{ }^{3} \mathrm{~J}_{\mathrm{H}, \mathrm{H}}=6.2 \mathrm{~Hz}\right), 4.44-4.42(4 \mathrm{H}, \mathrm{m}$, $\left.2 \operatorname{Ar}(\mathrm{C}=\mathrm{O}) \mathrm{OCH}_{2} \mathrm{CH}_{2} \mathrm{O}\right), \quad 3.79-3.77(4 \mathrm{H}, \mathrm{m}, \quad 2 \mathrm{Ar}(\mathrm{C}=\mathrm{O})-$ $\left.\mathrm{OCH}_{2} \mathrm{CH}_{2} \mathrm{O}\right), 3.70-3.61\left(16 \mathrm{H}, \mathrm{m}, 2 \mathrm{Ar}(\mathrm{C}=\mathrm{O}) \mathrm{O}\left(\mathrm{CH}_{2}\right)_{2} \mathrm{OCH}_{2} \mathrm{CH}_{2}\right.$, $2 \mathrm{Ar}(\mathrm{C}=\mathrm{O}) \mathrm{O}\left(\mathrm{CH}_{2}\right)_{2} \mathrm{OCH}_{2} \mathrm{CH}_{2}, 2 \mathrm{Ar}(\mathrm{C}=\mathrm{O}) \mathrm{O}\left(\mathrm{CH}_{2}\right)_{2} \mathrm{O}-$ $\left.\left.\left(\mathrm{CH}_{2}\right)_{2} \mathrm{OCH}_{2}\right), 2 \mathrm{Ar}(\mathrm{C}=\mathrm{O}) \mathrm{O}\left(\mathrm{CH}_{2}\right)_{2} \mathrm{O}\left(\mathrm{CH}_{2}\right)_{2} \mathrm{OCH}_{2} \mathrm{CH}_{2}\right), 2.85$ $\left(2 \mathrm{H}\right.$, sept, $\left.2(\mathrm{Ar}) \mathrm{CCHCHCCH}\left(\mathrm{CH}_{3}\right)_{2},{ }^{3} \mathrm{~J}_{\mathrm{H}, \mathrm{H}}=6.9 \mathrm{~Hz}\right), 1.85(6 \mathrm{H}, \mathrm{s}$, $\left.2 \mathrm{CH}_{3}(\mathrm{Ar}) \mathrm{CCHCHC}\right), \quad 1.10\left(12 \mathrm{H}, \mathrm{d}, 2(\mathrm{Ar}) \mathrm{CCHCHCCH}\left(\mathrm{CH}_{3}\right)_{2}\right.$, $\left.{ }^{3} J_{\mathrm{H}, \mathrm{H}}=6.9 \mathrm{~Hz}\right) \cdot{ }^{31} \mathrm{P} \mathrm{NMR}\left(\mathrm{CDCl}_{3}\right): \delta_{\mathrm{P}} 24.94(2 \mathrm{P}) \cdot{ }^{13} \mathrm{C} \mathrm{NMR}$ $\left(\mathrm{CDCl}_{3}\right): \delta_{\mathrm{C}} 166.1(2 \mathrm{C}, 2 \mathrm{O}(\mathrm{C}=\mathrm{O})(\mathrm{Ar}) \mathrm{CCHCHCP}), 139.4(2 \mathrm{C}, \mathrm{d}$, $\left.2 \mathrm{O}(\mathrm{C}=\mathrm{O})(\mathrm{Ar}) \mathrm{CCHCHCP},{ }^{1} \mathrm{~J}_{\mathrm{C}, \mathrm{P}}=44 \mathrm{~Hz}\right), 134.5(4 \mathrm{C}, \mathrm{d}, 4 \mathrm{O}(\mathrm{C}=$ O) $\left.(A r) \mathrm{CCHCHCP},{ }^{2} J_{\mathrm{C}, \mathrm{P}}=7 \mathrm{~Hz}\right), 134.4(8 \mathrm{C}, \mathrm{d}, 8 \mathrm{P}(\mathrm{Ar}) \mathrm{CCHCHCH}$, $\left.{ }^{2} J_{\mathrm{C}, \mathrm{P}}=9 \mathrm{~Hz}\right), 133.4\left(4 \mathrm{C}, \mathrm{d}, 4 \mathrm{P}(\mathrm{Ar}) \mathrm{CCHCHCH},{ }^{1} J_{\mathrm{C}, \mathrm{P}}=45 \mathrm{~Hz}\right), 131.4$ $\left(2 \mathrm{C}, \mathrm{d}, 2 \mathrm{O}(\mathrm{C}=\mathrm{O})(\mathrm{Ar}) \mathrm{CCHCHCP},{ }^{4} J_{\mathrm{C}, \mathrm{P}}=2 \mathrm{~Hz}\right), 130.7(4 \mathrm{C}, \mathrm{d}$, $\left.4 \mathrm{P}(\mathrm{Ar}) \mathrm{CCHCHCH},{ }^{4} \mathrm{~J}_{\mathrm{C}, \mathrm{P}}=2 \mathrm{~Hz}\right), 128.8(4 \mathrm{C}, \mathrm{d}, 4 \mathrm{O}(\mathrm{C}=\mathrm{O})(\mathrm{Ar})-$ $\left.\mathrm{CCHCHCP},{ }^{3} J_{\mathrm{C}, \mathrm{P}}=10 \mathrm{~Hz}\right), 128.3\left(8 \mathrm{C}, \mathrm{d}, 8 \mathrm{P}(\mathrm{Ar}) \mathrm{CCHCHCH},{ }^{3} J_{\mathrm{C}, \mathrm{P}}=\right.$ $10 \mathrm{~Hz}), 111.59,111.56\left(2 \mathrm{C}, 2 \mathrm{CH}_{3}(\mathrm{Ar}) \mathrm{CCHCHC}\right), 96.4$ (2C, $\left.2 \mathrm{CH}_{3}(\mathrm{Ar}) \mathrm{CCHCHC}\right), 89.12,89.09\left(4 \mathrm{C}, 4 \mathrm{CH}_{3}(\mathrm{Ar}) \mathrm{CCHCHC}\right)$, 87.48, 87.42 (4C, $\left.4 \mathrm{CH}_{3}(\mathrm{Ar}) \mathrm{CCHCHC}\right), 70.81,70.73,70.67$ (8C, $2(\mathrm{Ar})(\mathrm{C}=\mathrm{O}) \mathrm{O}\left(\mathrm{CH}_{2}\right)_{2} \mathrm{OCH}_{2}, 2(\mathrm{Ar})(\mathrm{C}=\mathrm{O}) \mathrm{O}\left(\mathrm{CH}_{2}\right)_{2} \mathrm{OCH}_{2} \mathrm{CH}_{2}, 2-$ $(A r)(\mathrm{C}=\mathrm{O}) \mathrm{O}\left(\mathrm{CH}_{2}\right)_{2} \mathrm{O}\left(\mathrm{CH}_{2}\right)_{2} \mathrm{OCH}_{2}, 2(\mathrm{Ar})(\mathrm{C}=\mathrm{O}) \mathrm{O}\left(\mathrm{CH}_{2}\right)_{2} \mathrm{O}-$ $\left.\left(\mathrm{CH}_{2}\right)_{2} \mathrm{OCH}_{2} \mathrm{CH}_{2}\right), 69.3\left(2 \mathrm{C}, 2(\mathrm{Ar})(\mathrm{C}=\mathrm{O}) \mathrm{OCH}_{2} \mathrm{CH}_{2} \mathrm{O}\right), 64.5$ $\left(2 \mathrm{C}, 2(\mathrm{Ar})(\mathrm{C}=\mathrm{O}) \mathrm{OCH}_{2} \mathrm{CH}_{2} \mathrm{O}\right), 30.4(2 \mathrm{C}, 2(\mathrm{Ar}) \mathrm{CHCHCCH}-$ $\left.\left(\mathrm{CH}_{3}\right)_{2}\right), 22.0\left(4 \mathrm{C}, 2(\mathrm{Ar}) \mathrm{CHCHCCH}\left(\mathrm{CH}_{3}\right)_{2}\right), 17.9\left(2 \mathrm{C}, 2 \mathrm{CH}_{3}(\mathrm{Ar})-\right.$ $\mathrm{CCHCH})$. ESI-MS(+). Calcd for $\mathrm{C}_{74} \mathrm{H}_{88} \mathrm{Cl}_{3} \mathrm{O}_{11} \mathrm{P}_{2} \mathrm{Ru}_{2}{ }^{+}: \mathrm{m} / z$ $1523.2949\left([\mathrm{M}-\mathrm{Cl}]^{+}\right)$. Found: $m / z$ 1523.2914. Calcd for $\mathrm{C}_{74} \mathrm{H}_{88} \mathrm{Cl}_{4} \mathrm{NaO}_{11} \mathrm{P}_{2} \mathrm{Ru}_{2}^{+}: m / z 1581.2535\left([\mathrm{M}+\mathrm{Na}]^{+}\right)$. Found: $m / z$ 1581.2789. UV-vis: $\lambda_{\max }=250$ and $370 \mathrm{~nm}$.

Compound $8 b$. According to the general procedure, $\left[\mathrm{Ru}\left(\eta^{6}-p\right.\right.$ cymene $) \mathrm{Cl}_{2} \mathrm{Cl}_{2}$ (0.30 g, $0.490 \mathrm{mmol}, 1$ equiv), $\mathrm{PPh}_{3}$ (0.26 g, 0.979 mmol, 2 equiv), and $\mathrm{CH}_{2} \mathrm{Cl}_{2}(12 \mathrm{~mL})$ were stirred for $42 \mathrm{~h}$ at RT. The product was isolated as a dark-red solid. Yield: $0.54 \mathrm{~g}, 0.950 \mathrm{mmol}, 9$. Elem anal. Calcd for $\mathrm{C}_{28} \mathrm{H}_{29} \mathrm{Cl}_{2} \mathrm{PRu}$ : C, 59.16; H, 5.14. Found: $\mathrm{C}$, 59.26; H, 4.90. ${ }^{1} \mathrm{H}$ NMR $\left(\mathrm{CDCl}_{3}\right): \delta_{\mathrm{H}} 7.77-7.85(6 \mathrm{H}, \mathrm{m}$, 6P $(A r) \mathrm{CCHCHCH}), 7.32-7.46(9 \mathrm{H}, \mathrm{m}, 6 \mathrm{P}(\mathrm{Ar}) \mathrm{CCHCHCH}, 3 \mathrm{P}-$ $(\mathrm{Ar}) \mathrm{CCHCHCH}), 5.19\left(2 \mathrm{H}, \mathrm{d}, 2 \mathrm{CH}_{3}(\mathrm{Ar}) \mathrm{CCHCHC},{ }^{3} \mathrm{~J}_{\mathrm{H}, \mathrm{H}}=6.2 \mathrm{~Hz}\right)$, $4.98\left(2 \mathrm{H}, \mathrm{d}, 2 \mathrm{CH}_{3}(\mathrm{Ar}) \mathrm{CCHCHC},{ }^{3} \mathrm{H}_{\mathrm{H}, \mathrm{H}}=6.2 \mathrm{~Hz}\right), 2.84(1 \mathrm{H}$, sept, $\left.(\mathrm{Ar}) \mathrm{CCHCHCCH}\left(\mathrm{CH}_{3}\right)_{2},{ }^{3} \mathrm{~J}_{\mathrm{H}, \mathrm{H}}=7.1 \mathrm{~Hz}\right), 1.86\left(3 \mathrm{H}, \mathrm{s}, \mathrm{CH}_{3}(\mathrm{Ar})-\right.$ CCHCHC), $1.09\left(6 \mathrm{H}, \mathrm{d},(\mathrm{Ar}) \mathrm{CCHCHCCH}\left(\mathrm{CH}_{3}\right)_{2},{ }^{3} J_{\mathrm{H}, \mathrm{H}}=7.1 \mathrm{~Hz}\right)$. ${ }^{31} \mathrm{P}$ NMR $\left(\mathrm{CDCl}_{3}\right): \delta_{\mathrm{P}} 24.18(1 \mathrm{P}) .{ }^{13} \mathrm{C} \mathrm{NMR}\left(\mathrm{CDCl}_{3}\right): \delta_{\mathrm{C}} 134.5(6 \mathrm{C}$, d, $\left.6 \mathrm{P}(\mathrm{Ar}) \mathrm{CCHCHCH},{ }^{2} J_{\mathrm{C}, \mathrm{P}}=9 \mathrm{~Hz}\right), 133.9(3 \mathrm{C}, \mathrm{d}, \quad 3 \mathrm{P}(\mathrm{Ar})$ $\left.\mathrm{CCHCHCH},{ }^{1} J_{\mathrm{C}, \mathrm{P}}=46 \mathrm{~Hz}\right), 130.3\left(3 \mathrm{C}, \mathrm{d}, 3 \mathrm{P}(\mathrm{Ar}) \mathrm{CCHCHCH},{ }^{4} J_{\mathrm{C}, \mathrm{P}}\right.$ $=2 \mathrm{~Hz}), 128.1\left(8 \mathrm{C}, \mathrm{d}, 8 \mathrm{P}(\mathrm{Ar}) \mathrm{CCHCHCH},{ }^{3} J_{\mathrm{C}, \mathrm{P}}=10 \mathrm{~Hz}\right), 111.20$, 111.17 (1C, $\left.\mathrm{CH}_{3}(\mathrm{Ar}) \mathrm{CCHCHC}\right), 96.1\left(1 \mathrm{C}, 2 \mathrm{CH}_{3}(\mathrm{Ar}) \mathrm{CCHCHC}\right)$, 89.18, $89.15\left(2 \mathrm{C}, 2 \mathrm{CH}_{3}(\mathrm{Ar}) \mathrm{CCHCHC}\right), 87.28,87.23\left(2 \mathrm{C}, 2 \mathrm{CH}_{3}(\mathrm{Ar})-\right.$ $\mathrm{CCHCHC}), 30.3\left(1 \mathrm{C},(\mathrm{Ar}) \mathrm{CHCHCCH}\left(\mathrm{CH}_{3}\right)_{2}\right), 22.0(2 \mathrm{C},(\mathrm{Ar})-$ $\left.\mathrm{CHCHCCH}\left(\mathrm{CH}_{3}\right)_{2}\right), 17.8 \quad\left(1 \mathrm{C}, \mathrm{CH}_{3}(\mathrm{Ar}) \mathrm{CCHCH}\right)$. ESI-MS(+). Calcd for $\mathrm{C}_{28} \mathrm{H}_{29} \mathrm{PRu}^{2+}: \mathrm{m} / z$ 498.1050 $\left([\mathrm{M}-2 \mathrm{Cl}]^{+}\right)$. Found: $\mathrm{m} / z$ 498.1009. Calcd for $\mathrm{C}_{28} \mathrm{H}_{29} \mathrm{ClPRu}^{+}: \mathrm{m} / z 533.0739\left([\mathrm{M}-\mathrm{Cl}]^{+}\right)$. Found: $m / z$ 533.0772.

Compound $9 b$. According to the general procedure, $\left[\mathrm{Ru}\left(\eta^{6}-p\right.\right.$ cymene $\mathrm{Cl}_{2} \mathrm{Cl}_{2}(0.30 \mathrm{~g}, 0.980 \mathrm{mmol}, 1$ equiv), 4(diphenylphosphanyl)benzoic acid $(0.30 \mathrm{~g}, 0.980 \mathrm{mmol}, 1$ equiv), and $\mathrm{CH}_{2} \mathrm{Cl}_{2}(12 \mathrm{~mL})$, were stirred for $42 \mathrm{~h}$ at RT. The product was isolated as a dark-red solid. Yield: $0.59 \mathrm{~g}, 0.961 \mathrm{mmol}, 98 \%$. Elem anal. Calcd for $\mathrm{C}_{29} \mathrm{H}_{29} \mathrm{Cl}_{2} \mathrm{O}_{2} \mathrm{PRu} .{ }^{1}{ }_{2} \mathrm{CHCl}_{3}$ : C, 52.71; H, 4.42. Found: C, $52.73 ; \mathrm{H}, 4.67 . \mathrm{CHCl}_{3}$ originates from the recrystallization process. ${ }^{1} \mathrm{H}$ NMR $\left(\mathrm{CDCl}_{3}\right): \delta_{\mathrm{H}}-7.97-8.00(4 \mathrm{H}, \mathrm{m}, \quad 2 \mathrm{O}(\mathrm{C}=\mathrm{O})(\mathrm{Ar})$ CCHCHCP $), 7.78-7.84(4 \mathrm{H}, \mathrm{m}, 4 \mathrm{P}(\mathrm{Ar}) \mathrm{CCHCHCH}), 7.36-7.45$ $(6 \mathrm{H}, \mathrm{m}, 4 \mathrm{P}(\mathrm{Ar}) \mathrm{CCHCHCH}, 2 \mathrm{P}(A r) \mathrm{CCHCHCH}), 5.22(2 \mathrm{H}, \mathrm{d}$, $\left.2 \mathrm{CH}_{3}(\mathrm{Ar}) \mathrm{CCHCHC},{ }^{3} J_{\mathrm{H}, \mathrm{H}}=6.1 \mathrm{~Hz}\right), 4.99\left(2 \mathrm{H}, \mathrm{d}, 2 \mathrm{CH}_{3}(A r)-\right.$ $\left.\mathrm{CCHCHC},{ }^{3} J_{\mathrm{H}, \mathrm{H}}=6.1 \mathrm{~Hz}\right), 2.84(1 \mathrm{H}$, sept, $(A r)-$ $\left.\mathrm{CCHCHCCH}\left(\mathrm{CH}_{3}\right)_{2},{ }^{3} J_{\mathrm{H}, \mathrm{H}}=6.9 \mathrm{~Hz}\right), 1.85\left(3 \mathrm{H}, \mathrm{s}, \mathrm{CH}_{3}(\mathrm{Ar})-\right.$ $\mathrm{CCHCHC}), \quad 1.10\left(6 \mathrm{H}, \mathrm{d},(\mathrm{Ar}) \mathrm{CCHCHCCH}\left(\mathrm{CH}_{3}\right)_{2},{ }^{3} J_{\mathrm{H}, \mathrm{H}}=6.9\right.$ $\mathrm{Hz}) .{ }^{31} \mathrm{P}$ NMR $\left(\mathrm{CDCl}_{3}\right): \delta_{\mathrm{P}} 25.27(1 \mathrm{P}) .{ }^{13} \mathrm{C} \mathrm{NMR}\left(\mathrm{CDCl}_{3}\right): \delta_{\mathrm{C}} 171.1$ $(1 \mathrm{C}, \mathrm{O}(C=\mathrm{O})(A r) \mathrm{CCHCHCP}), 140.4 \quad(1 \mathrm{C}, \mathrm{d}, \mathrm{O}(\mathrm{C}=\mathrm{O})(A r)-$ CСНCHCP, $\left.{ }^{1} J_{\mathrm{C}, \mathrm{P}}=43 \mathrm{~Hz}\right), 134.5(2 \mathrm{C}, \mathrm{d}, 2 \mathrm{O}(\mathrm{C}=\mathrm{O})(A r)-$ $\left.\mathrm{CCHCHCP},{ }^{2} J_{\mathrm{C}, \mathrm{P}}=11 \mathrm{~Hz}\right), 134.4\left(4 \mathrm{C}, \mathrm{d}, 4 \mathrm{P}(\mathrm{Ar}) \mathrm{CCHCHCH},{ }^{2} J_{\mathrm{C}, \mathrm{P}}\right.$ $=10 \mathrm{~Hz}), 133.3\left(2 \mathrm{C}, \mathrm{d}, 2 \mathrm{P}(\mathrm{Ar}) \mathrm{CCHCHCH},{ }^{1} \mathrm{~J}_{\mathrm{C}, \mathrm{P}}=45 \mathrm{~Hz}\right), 130.7$ $\left(2 \mathrm{C}, \mathrm{d}, 2 \mathrm{P}(\mathrm{Ar}) \mathrm{CCHCHCH},{ }^{4} J_{\mathrm{C}, \mathrm{P}}=1 \mathrm{~Hz}\right), 130.5(1 \mathrm{C}, \mathrm{d}, \mathrm{O}(\mathrm{C}=$ O) $\left.(\mathrm{Ar}) \mathrm{CCHCHCP},{ }^{4} J_{\mathrm{C}, \mathrm{P}}=2 \mathrm{~Hz}\right), 129.3(2 \mathrm{C}, \mathrm{d}, 2 \mathrm{O}(\mathrm{C}=\mathrm{O})(\mathrm{Ar})-$ $\left.\mathrm{CCHCHCP},{ }^{3} J_{\mathrm{CP}}=10 \mathrm{~Hz}\right), 128.3\left(4 \mathrm{C}, \mathrm{d}, 4 \mathrm{P}(\mathrm{Ar}) \mathrm{CCHCHCH},{ }^{3} J_{\mathrm{CP}}=\right.$ $10 \mathrm{~Hz}), 111.57,111.61\left(1 \mathrm{C}, \mathrm{CH}_{3}(\mathrm{Ar}) \mathrm{CCHCHC}\right), 96.5\left(1 \mathrm{C}, \mathrm{CH}_{3}(\mathrm{Ar})\right.$ CCHCHC), 89.06, $89.03\left(2 \mathrm{C}, 2 \mathrm{CH}_{3}(\mathrm{Ar}) \mathrm{CCHCHC}\right), 87.51,87.46$ $\left(2 \mathrm{C}, 2 \mathrm{CH}_{3}(\mathrm{Ar}) \mathrm{CCHCHC}\right), 30.4\left(1 \mathrm{C},(\mathrm{Ar}) \mathrm{CHCHCCH}\left(\mathrm{CH}_{3}\right)_{2}\right), 22.0$ (2C, $\left.(\mathrm{Ar}) \mathrm{CHCHCCH}\left(\mathrm{CH}_{3}\right)_{2}\right), 17.9\left(1 \mathrm{C}, \mathrm{CH}_{3}(\mathrm{Ar}) \mathrm{CCHCH}\right)$. ESI$\mathrm{MS}(+)$. Calcd for $\mathrm{C}_{29} \mathrm{H}_{29} \mathrm{O}_{2} \mathrm{PRu}^{+}: \mathrm{m} / z 542.0949\left([\mathrm{M}-2 \mathrm{Cl}]^{+}\right)$. Found: $m / z$ 542.0914. Calcd for $\mathrm{C}_{29} \mathrm{H}_{29} \mathrm{ClO}_{2} \mathrm{PRu}^{+}: m / z$ 577.0637 ([M $\left.-\mathrm{Cl}]^{+}\right)$. Found: $m / z$ 577.0648. Calcd for $\mathrm{C}_{29} \mathrm{H}_{29} \mathrm{Cl}_{2} \mathrm{NaO}_{2} \mathrm{PRu}^{+}: m / z$ $635.0223\left([\mathrm{M}+\mathrm{Na}]^{+}\right.$. Found: $m / z$ 635.0228).

General Procedure for the Synthesis of the Digold Intermediates $1 c-7 c$. The appropriate ligand, $1 \mathbf{a}-7 \mathbf{a}$ ( 1 equiv), dissolved in dry $\mathrm{CH}_{2} \mathrm{Cl}_{2}(15 \mathrm{~mL}$ ), was added to a solution of $\mathrm{AuCl}(\mathrm{tht})$ (2 equiv) in dry $\mathrm{CH}_{2} \mathrm{Cl}_{2}(10 \mathrm{~mL})$ at $0{ }^{\circ} \mathrm{C}$ under $\mathrm{N}_{2}$. The reaction mixture was stirred at RT for $6 \mathrm{~h}$, and the reaction evolution was monitored by ${ }^{1} \mathrm{H}$ and ${ }^{31} \mathrm{P}$ NMR $\left(\mathrm{CDCl}_{3}\right)$. The reaction mixture was concentrated under reduced pressure, and the product was washed with hexane $(5 \times 25$ $\mathrm{mL})$ and resolubilized in $\mathrm{CH}_{2} \mathrm{Cl}_{2}(50 \mathrm{~mL})$, before being concentrated and further dried under high vacuum. The product was isolated as a white solid and stored at $-20{ }^{\circ} \mathrm{C}$ in the dark.

Compound 1c. According to the general procedure, $\mathrm{AuCl}($ tht $)$ ( $0.188 \mathrm{~g}, 0.588 \mathrm{mmol}, 2$ equiv), $1 \mathrm{a}(0.188 \mathrm{~g}, 0.294 \mathrm{mmol}, 1$ equiv), and $\mathrm{CH}_{2} \mathrm{Cl}_{2}(35 \mathrm{~mL})$ were stirred for $6 \mathrm{~h}$ at $\mathrm{RT}$. The product was isolated as a white solid. Yield: $0.319 \mathrm{~g}, 0.289 \mathrm{mmol}, 98 \%$. Elem anal. Calcd for $\mathrm{C}_{40} \mathrm{H}_{32} \mathrm{Au}_{2} \mathrm{Cl}_{2} \mathrm{O}_{4} \mathrm{P}_{2}$ : C, 43.54; H, 2.92. Found: $\mathrm{C}, 43.55 ; \mathrm{H}$, 3.02. ${ }^{1} \mathrm{H}$ NMR $\left(\mathrm{CDCl}_{3}\right): \delta_{\mathrm{H}} 8.07-8.12(4 \mathrm{H}, \mathrm{m}, 4 \mathrm{O}(\mathrm{C}=\mathrm{O})(A r)-$ CCHCHCP, $\left.{ }^{3} J_{\mathrm{H}, \mathrm{H}}=8.4 \mathrm{~Hz},{ }^{4} J_{\mathrm{H}, \mathrm{H}}=2.1 \mathrm{~Hz}\right), 7.45-7.60(24 \mathrm{H}, \mathrm{m}$, $4 \mathrm{O}(\mathrm{C}=\mathrm{O})(A r) \mathrm{CCHCHCP}, 8 \mathrm{P}(A r) \mathrm{CCHCHCH}, 8 \mathrm{P}(A r)-$ $\mathrm{CCHCHCH}, 4 \mathrm{P}(\mathrm{Ar}) \mathrm{CCHCHCH}), 4.68(4 \mathrm{H}, \mathrm{s}, \quad \operatorname{Ar}(\mathrm{C}=\mathrm{O}) \mathrm{O}-$ $\left.\left(\mathrm{CH}_{2}\right)_{2} \mathrm{O}\right) \cdot{ }^{31} \mathrm{P}$ NMR $\left(\mathrm{CDCl}_{3}\right): \delta_{\mathrm{P}} 33.14(2 \mathrm{P}) .{ }^{13} \mathrm{C}$ NMR $\left(\mathrm{CDCl}_{3}\right)$ : $\delta_{\mathrm{C}} 165.3(2 \mathrm{C}, 2 \mathrm{O}(\mathrm{C}=\mathrm{O})(\mathrm{Ar}) \mathrm{CCHCHCP}), 134.9(2 \mathrm{C}, \mathrm{d}, 2 \mathrm{O}(\mathrm{C}=$ O) $\left.(A r) \mathrm{CCHCHCP},{ }^{1} J_{\mathrm{C}, \mathrm{P}}=59 \mathrm{~Hz}\right), 134.3(8 \mathrm{C}, \mathrm{d}, 8 \mathrm{P}(\mathrm{Ar}) \mathrm{CCHCHCH}$, $\left.{ }^{2} J_{\mathrm{C}, \mathrm{P}}=14 \mathrm{~Hz}\right), 134.1\left(4 \mathrm{C}, \mathrm{d}, 4 \mathrm{O}(\mathrm{C}=\mathrm{O})(\mathrm{Ar}) \mathrm{CCHCHCP},{ }^{2} J_{\mathrm{C}, \mathrm{P}}=14\right.$ $\mathrm{Hz}), 132.9\left(2 \mathrm{C}, \mathrm{d}, 2 \mathrm{O}(\mathrm{C}=\mathrm{O})(\mathrm{Ar}) \mathrm{CCHCHCP},{ }^{4} J_{\mathrm{C}, \mathrm{P}}=3 \mathrm{~Hz}\right), 132.5$ $\left(4 \mathrm{C}, \mathrm{d}, 4 \mathrm{P}(\mathrm{Ar}) \mathrm{CCHCHCH},{ }^{4} \mathrm{~J}_{\mathrm{C}, \mathrm{P}}=3 \mathrm{~Hz}\right), 130.2(4 \mathrm{C}, \mathrm{d}, 4 \mathrm{O}(\mathrm{C}=$ O) $\left.(\mathrm{Ar}) \mathrm{CCHCHCP},{ }^{3} J_{\mathrm{C}, \mathrm{P}}=12 \mathrm{~Hz}\right), 129.6(8 \mathrm{C}, \mathrm{d}, 8 \mathrm{P}(\mathrm{Ar}) \mathrm{CCHCHCH}$, $\left.{ }^{3} J_{\mathrm{C}, \mathrm{P}}=12 \mathrm{~Hz}\right), 127.9\left(4 \mathrm{C}, \mathrm{d}, 4 \mathrm{P}(\mathrm{Ar}) \mathrm{CCHCHCH},{ }^{1} J_{\mathrm{C}, \mathrm{P}}=63 \mathrm{~Hz}\right), 63.3$ $\left(2 \mathrm{C}, \quad 2(\mathrm{Ar})(\mathrm{C}=\mathrm{O}) \mathrm{O}\left(\mathrm{CH}_{2}\right)_{2} \mathrm{O}\right)$. ESI-MS $(+)$. Calcd for $\mathrm{C}_{40} \mathrm{H}_{32} \mathrm{Au}_{2} \mathrm{ClO}_{4} \mathrm{P}_{2}^{+}: m / z 1067.0795\left([\mathrm{M}-\mathrm{Cl}]^{+}\right)$. Found: $m / z$ 
1067.0825. Calcd for $\mathrm{C}_{40} \mathrm{H}_{32} \mathrm{Au}_{2} \mathrm{Cl}_{2} \mathrm{NaO}_{4} \mathrm{P}_{2}^{+}: m / z 1125.0382([\mathrm{M}+$ $\mathrm{Na}]^{+}$). Found: $m / z 1125.0388$.

Compound $2 \mathrm{c}$. According to the general procedure, $\mathrm{AuCl}(\mathrm{tht})$ ( $0.235 \mathrm{~g}, 0.735 \mathrm{mmol}, 2$ equiv), $2 \mathrm{a}(0.250 \mathrm{~g}, 0.366 \mathrm{mmol}, 1$ equiv), and $\mathrm{CH}_{2} \mathrm{Cl}_{2}(35 \mathrm{~mL})$ were stirred for $6 \mathrm{~h}$ at $\mathrm{RT}$. The product was isolated as a white solid. Yield: $0.403 \mathrm{~g}, 0.351 \mathrm{mmol}, 96$. Elem anal. Calcd for $\mathrm{C}_{42} \mathrm{H}_{36} \mathrm{Au}_{2} \mathrm{Cl}_{2} \mathrm{O}_{5} \mathrm{P}_{2}$ : C, 43.96; H, 3.16. Found: $\mathrm{C}, 43.83 ; \mathrm{H}, 3.23$. ${ }^{1} \mathrm{H}$ NMR $\left(\mathrm{CDCl}_{3}\right): \delta_{\mathrm{H}} 8.06-8.10(4 \mathrm{H}, \mathrm{m}, 4 \mathrm{O}(\mathrm{C}=\mathrm{O})(\mathrm{Ar}) \mathrm{CCHCHCP}$, $\left.{ }^{3} J_{\mathrm{H}, \mathrm{H}}=8.2 \mathrm{~Hz},{ }^{4} J_{\mathrm{H}, \mathrm{H}}=2 \mathrm{~Hz}\right), 7.44-7.60(24 \mathrm{H}, \mathrm{m}, 4 \mathrm{O}(\mathrm{C}=$ $\mathrm{O})(A r) \mathrm{CCHCHCP}, 8 \mathrm{P}(A r) \mathrm{CCHCHCH}, 8 \mathrm{P}(A r) \mathrm{CCHCHCH}, 4 \mathrm{P}-$ $(\mathrm{Ar}) \mathrm{CCHCHCH}), 4.48-4.50\left(4 \mathrm{H}, \mathrm{m}, 2 \mathrm{Ar}(\mathrm{C}=\mathrm{O}) \mathrm{OCH}_{2} \mathrm{CH}_{2} \mathrm{O}\right)$, 3.85-3.87 (4H, m, $\left.2 \mathrm{Ar}(\mathrm{C}=\mathrm{O}) \mathrm{OCH}_{2} \mathrm{CH}_{2} \mathrm{O}\right) .{ }^{31} \mathrm{P}$ NMR $\left(\mathrm{CDCl}_{3}\right)$ : $\delta_{\mathrm{p}} 33.02(2 \mathrm{P}) .{ }^{13} \mathrm{C}$ NMR $\left(\mathrm{CDCl}_{3}\right): \delta_{\mathrm{C}} 165.4(2 \mathrm{C}, 2 \mathrm{O}(\mathrm{C}=$ $\mathrm{O})(\mathrm{Ar}) \mathrm{CCHCHCP}), 134.6(2 \mathrm{C}, \mathrm{d}, 2 \mathrm{O}(\mathrm{C}=\mathrm{O})(\mathrm{Ar}) \mathrm{CCHCHCP}$, $\left.{ }^{1} J_{\mathrm{C}, \mathrm{P}}=62 \mathrm{~Hz}\right), 134.3\left(8 \mathrm{C}, \mathrm{d}, 8 \mathrm{P}(A r) \mathrm{CCHCHCH},{ }^{2} J_{\mathrm{C}, \mathrm{P}}=14 \mathrm{~Hz}\right)$, $134.0\left(4 \mathrm{C}, \mathrm{d}, 4 \mathrm{O}(\mathrm{C}=\mathrm{O})(\mathrm{Ar}) \mathrm{CCHCHCP},{ }^{2} J_{\mathrm{C}, \mathrm{P}}=14 \mathrm{~Hz}\right), 133.2(2 \mathrm{C}$, $\left.\mathrm{d}, 2 \mathrm{O}(\mathrm{C}=\mathrm{O})(A r) C \mathrm{CHCHCP},{ }^{4} J_{\mathrm{C}, \mathrm{P}}=2 \mathrm{~Hz}\right), 132.5(4 \mathrm{C}, \mathrm{d}$, $\left.4 \mathrm{P}(\mathrm{Ar}) \mathrm{CCHCHCH},{ }^{4} J_{\mathrm{C}, \mathrm{P}}=2 \mathrm{~Hz}\right), 130.1(4 \mathrm{C}, \mathrm{d}, 4 \mathrm{O}(\mathrm{C}=\mathrm{O})(A r)-$ $\left.\mathrm{CCHCHCP},{ }^{3} J_{\mathrm{C}, \mathrm{P}}=12 \mathrm{~Hz}\right), 129.6\left(8 \mathrm{C}, \mathrm{d}, 8 \mathrm{P}(\mathrm{Ar}) \mathrm{CCHCHCH},{ }^{3} J_{\mathrm{C}, \mathrm{P}}=\right.$ $12 \mathrm{~Hz}), 128.0\left(4 \mathrm{C}, \mathrm{d}, 4 \mathrm{P}(\mathrm{Ar}) \mathrm{CCHCHCH},{ }_{\mathrm{C}, \mathrm{P}}=63 \mathrm{~Hz}\right), 69.2(2 \mathrm{C}$, $\left.2(\mathrm{Ar})(\mathrm{C}=\mathrm{O}) \mathrm{OCH}_{2} \mathrm{CH}_{2} \mathrm{O}\right), 64.6\left(2 \mathrm{C}, 2(\mathrm{Ar})(\mathrm{C}=\mathrm{O}) \mathrm{OCH}_{2} \mathrm{CH}_{2} \mathrm{O}\right)$. ESI-MS(+). Calcd for $\mathrm{C}_{42} \mathrm{H}_{36} \mathrm{Au}_{2} \mathrm{ClO}_{5} \mathrm{P}_{2}^{+}: \mathrm{m} / z 1111.1058$ ([M $\left.\mathrm{Cl}]^{+}\right)$. Found: $m / z$ 1111.1104. Calcd for $\mathrm{C}_{42} \mathrm{H}_{36} \mathrm{Au}_{2} \mathrm{Cl}_{2} \mathrm{NaO}_{5} \mathrm{P}_{2}^{+}: m / z$ $1169.0644\left([\mathrm{M}+\mathrm{Na}]^{+}\right)$.. Found: $m / z 1169.0690$

Compound 3c. According to the general procedure, $\mathrm{AuCl}(\mathrm{tht})$ ( $0.221 \mathrm{~g}, 0.692 \mathrm{mmol}, 2$ equiv), $3 \mathrm{a}(0.250 \mathrm{~g}, 0.344 \mathrm{mmol}, 1$ equiv $)$, and $\mathrm{CH}_{2} \mathrm{Cl}_{2}(35 \mathrm{~mL})$ were stirred $6 \mathrm{~h}$ at $\mathrm{RT}$. The product was isolated as a white solid. Yield: $0.406 \mathrm{~g}, 0.341 \mathrm{mmol}, 98 \%$. Elem anal. Calcd for $\mathrm{C}_{44} \mathrm{H}_{40} \mathrm{Au}_{2} \mathrm{Cl}_{2} \mathrm{O}_{6} \mathrm{P}_{2}$ : C, 44.35; H, 3.38. Found: C, 44.30; $\mathrm{H}, 3.51 .{ }^{1} \mathrm{H}$ NMR $\left(\mathrm{CDCl}_{3}\right): \delta_{\mathrm{H}} 8.07-8.11(4 \mathrm{H}, \mathrm{m}, 4 \mathrm{O}(\mathrm{C}=\mathrm{O})(A r) \mathrm{CCHCHCP}$, $\left.{ }^{3} J_{\mathrm{H}, \mathrm{H}}=8.4 \mathrm{~Hz},{ }^{4} \mathrm{~J}_{\mathrm{H}, \mathrm{H}}=2.2 \mathrm{~Hz}\right), 7.45-7.60(24 \mathrm{H}, \mathrm{m}, 4 \mathrm{O}(\mathrm{C}=$ $\mathrm{O})(A r) \mathrm{CCHCHCP}, 8 \mathrm{P}(A r) \mathrm{CCHCHCH}, 8 \mathrm{P}(A r) \mathrm{CCHCHCH}, 4 \mathrm{P}-$ $(\mathrm{Ar}) \mathrm{CCHCHCH}), 4.44-4.48\left(4 \mathrm{H}, \mathrm{m}, 2 \mathrm{Ar}(\mathrm{C}=\mathrm{O}) \mathrm{OCH}_{2} \mathrm{CH}_{2} \mathrm{O}\right)$, 3.79-3.83 $\left(4 \mathrm{H}, \mathrm{m}, 2 \mathrm{Ar}(\mathrm{C}=\mathrm{O}) \mathrm{OCH}_{2} \mathrm{CH}_{2} \mathrm{O}\right), 3.69(4 \mathrm{H}, \mathrm{s}, 2 \mathrm{Ar}(\mathrm{C}=$ O) $\left.\mathrm{O}\left(\mathrm{CH}_{2}\right)_{2} \mathrm{OCH}_{2}\right) \cdot{ }^{31} \mathrm{P}$ NMR $\left(\mathrm{CDCl}_{3}\right): \delta_{\mathrm{P}} 33.07(2 \mathrm{P}) .{ }^{13} \mathrm{C}$ NMR $\left(\mathrm{CDCl}_{3}\right): \delta_{\mathrm{C}} 165.4(2 \mathrm{C}, 2 \mathrm{O}(\mathrm{C}=\mathrm{O})(\mathrm{Ar}) \mathrm{CCHCHCP}), 134.5(2 \mathrm{C}, \mathrm{d}$, $\left.2 \mathrm{O}(\mathrm{C}=\mathrm{O})(A r) \mathrm{CCHCHCP},{ }^{1} J_{\mathrm{C}, \mathrm{P}}=60 \mathrm{~Hz}\right), 134.3(8 \mathrm{C}, \mathrm{d}, 8 \mathrm{P}(A r)-$ $\left.\mathrm{CCHCHCH},{ }^{2} J_{\mathrm{C}, \mathrm{P}}=14 \mathrm{~Hz}\right), 134.2(4 \mathrm{C}, \mathrm{d}, 4 \mathrm{O}(\mathrm{C}=\mathrm{O})(\mathrm{Ar})-$ $\left.\mathrm{CCHCHCP},{ }^{2} J_{\mathrm{C}, \mathrm{P}}=14 \mathrm{~Hz}\right), 133.3(2 \mathrm{C}, \mathrm{d}, 2 \mathrm{O}(\mathrm{C}=\mathrm{O})(A r)$ $\left.\mathrm{CCHCHCP},{ }^{4} J_{\mathrm{C}, \mathrm{P}}=3 \mathrm{~Hz}\right), 132.5\left(4 \mathrm{C}, \mathrm{d}, 4 \mathrm{P}(\mathrm{Ar}) \mathrm{CCHCHCH},{ }^{4} J_{\mathrm{C}, \mathrm{P}}\right.$ $=2 \mathrm{~Hz}), 130.2\left(4 \mathrm{C}, \mathrm{d}, 4 \mathrm{O}(\mathrm{C}=\mathrm{O})(A r) \mathrm{CCHCHCP},{ }^{3} J_{\mathrm{C}, \mathrm{P}}=12 \mathrm{~Hz}\right)$, $129.5\left(8 \mathrm{C}, \mathrm{d}, 8 \mathrm{P}(\mathrm{Ar}) \mathrm{CCHCHCH},{ }^{3} J_{\mathrm{C}, \mathrm{P}}=12 \mathrm{~Hz}\right), 128.0(4 \mathrm{C}, \mathrm{d}$, $\left.4 \mathrm{P}(\mathrm{Ar}) \mathrm{CCHCHCH},{ }^{1} J_{\mathrm{C}, \mathrm{P}}=63 \mathrm{~Hz}\right), 70.8(2 \mathrm{C}, 2(\mathrm{Ar})(\mathrm{C}=\mathrm{O}) \mathrm{O}-$ $\left.\left(\mathrm{CH}_{2}\right)_{2} \mathrm{OCH}_{2}\right), 69.3\left(2 \mathrm{C}, 2(\mathrm{Ar})(\mathrm{C}=\mathrm{O}) \mathrm{OCH}_{2} \mathrm{CH}_{2} \mathrm{O}\right), 64.7(2 \mathrm{C}$, $\left.2(\mathrm{Ar})(\mathrm{C}=\mathrm{O}) \mathrm{OCH}_{2} \mathrm{CH}_{2} \mathrm{O}\right)$. ESI-MS $(+)$. Calcd for $\mathrm{C}_{44} \mathrm{H}_{40} \mathrm{Au}_{2} \mathrm{ClO}_{6} \mathrm{P}_{2}^{+}: m / z 1155.1320\left([\mathrm{M}-\mathrm{Cl}]^{+}\right)$. Found: $m / z$ 1155.1376. Calcd for $\mathrm{C}_{44} \mathrm{H}_{40} \mathrm{Au}_{2} \mathrm{Cl}_{2} \mathrm{NaO}_{6} \mathrm{P}_{2}^{+}: m / z 1213.0906([\mathrm{M}+$ $\mathrm{Na}]^{+}$). Found: $m / z 1213.0951$.

Compound 4c. According to the general procedure, $\mathrm{AuCl}(\mathrm{tht})$ ( $0.208 \mathrm{~g}, 0.651 \mathrm{mmol}, 2$ equiv), $4 \mathrm{a}(0.250 \mathrm{~g}, 0.324 \mathrm{mmol}, 1$ equiv $)$, and $\mathrm{CH}_{2} \mathrm{Cl}_{2}(35 \mathrm{~mL})$ were stirred $6 \mathrm{~h}$ at $\mathrm{RT}$. The product was isolated as a white solid. Yield: $0.389 \mathrm{~g}, 0.315 \mathrm{mmol}, 97 \%$. Elem anal. Calcd for $\mathrm{C}_{46} \mathrm{H}_{44} \mathrm{Au}_{2} \mathrm{Cl}_{2} \mathrm{O}_{7} \mathrm{P}_{2}: \mathrm{C}, 44.71 ; \mathrm{H}, 3.59$. Found: $\mathrm{C}, 44.78 ; \mathrm{H}, 3.57 .{ }^{1} \mathrm{H}$ NMR $\left(\mathrm{CDCl}_{3}\right): \delta_{\mathrm{H}} 8.08-8.12(4 \mathrm{H}, \mathrm{m}, 4 \mathrm{O}(\mathrm{C}=\mathrm{O})(\mathrm{Ar}) \mathrm{CCHCHCP}$, $\left.{ }^{3} J_{\mathrm{H}, \mathrm{H}}=8.4 \mathrm{~Hz},{ }^{4} J_{\mathrm{H}, \mathrm{H}}=2 \mathrm{~Hz}\right), 7.46-7.60(24 \mathrm{H}, \mathrm{m}, 4 \mathrm{O}(\mathrm{C}=$ $\mathrm{O})(A r) \mathrm{CCHCHCP}, 8 \mathrm{P}(A r) \mathrm{CCHCHCH}, 8 \mathrm{P}(A r) \mathrm{CCHCHCH}, 4 \mathrm{P}-$ $(\mathrm{Ar}) \mathrm{CCHCHCH}), 4.46-4.49\left(4 \mathrm{H}, \mathrm{m}, 2 \mathrm{Ar}(\mathrm{C}=\mathrm{O}) \mathrm{OCH}_{2} \mathrm{CH}_{2} \mathrm{O}\right)$, 3.79-3.82 (4H, m, $\left.2 \mathrm{Ar}(\mathrm{C}=\mathrm{O}) \mathrm{OCH}_{2} \mathrm{CH}_{2} \mathrm{O}\right), 3.61-3.72(8 \mathrm{H}, \mathrm{m}$, $\left.2 A r(\mathrm{C}=\mathrm{O}) \mathrm{O}\left(\mathrm{CH}_{2}\right)_{2} \mathrm{O}\left(\mathrm{CH}_{2}\right)_{2}\right) .{ }^{31} \mathrm{P}$ NMR $\left(\mathrm{CDCl}_{3}\right): \delta_{\mathrm{P}} 33.01(2 \mathrm{P})$. ${ }^{13} \mathrm{C}$ NMR $\left(\mathrm{CDCl}_{3}\right): \delta_{\mathrm{C}} 165.4(2 \mathrm{C}, 2 \mathrm{O}(\mathrm{C}=\mathrm{O})(\mathrm{Ar}) \mathrm{CCHCHCP})$, $134.5\left(2 \mathrm{C}, \mathrm{d}, 2 \mathrm{O}(\mathrm{C}=\mathrm{O})(\mathrm{Ar}) \mathrm{CCHCHCP},{ }^{1} J_{\mathrm{C}, \mathrm{P}}=58 \mathrm{~Hz}\right), 134.3(8 \mathrm{C}$, d, $\left.8 \mathrm{P}(A r) \mathrm{CCHCHCH},{ }^{2} J_{\mathrm{C}, \mathrm{P}}=14 \mathrm{~Hz}\right), 134.0(4 \mathrm{C}, \mathrm{d}, 4 \mathrm{O}(\mathrm{C}=$ O) $\left.(A r) \mathrm{CCHCHCP},{ }^{2} J_{\mathrm{C}, \mathrm{P}}=14 \mathrm{~Hz}\right), 133.3(2 \mathrm{C}, \mathrm{d}, 2 \mathrm{O}(\mathrm{C}=\mathrm{O})(\mathrm{Ar})$ CCHCHCP, $\left.{ }^{4} J_{\mathrm{C}, \mathrm{P}}=2 \mathrm{~Hz}\right), 132.4\left(4 \mathrm{C}, \mathrm{d}, 4 \mathrm{P}(\mathrm{Ar}) \mathrm{CCHCHCH},{ }^{4} J_{\mathrm{C}, \mathrm{P}}=2\right.$ $\mathrm{Hz}), 130.1\left(4 \mathrm{C}, \mathrm{d}, 4 \mathrm{O}(\mathrm{C}=\mathrm{O})(A r) \mathrm{CCHCHCP},{ }^{3} \mathrm{~J}_{\mathrm{C}, \mathrm{P}}=12 \mathrm{~Hz}\right), 129.5$ $\left(8 \mathrm{C}, \mathrm{d}, 8 \mathrm{P}(\mathrm{Ar}) \mathrm{CCHCHCH},{ }^{3} \mathrm{~J}_{\mathrm{C}, \mathrm{P}}=12 \mathrm{~Hz}\right), 127.9(4 \mathrm{C}, \mathrm{d}, 4 \mathrm{P}(A r)$ $\left.\mathrm{CCHCHCH},{ }^{1} J_{\mathrm{C}, \mathrm{P}}=63 \mathrm{~Hz}\right), 70.7\left(4 \mathrm{C}, 2(\mathrm{Ar})(\mathrm{C}=\mathrm{O}) \mathrm{O}\left(\mathrm{CH}_{2}\right)_{2} \mathrm{OCH}_{2}\right.$, $\left.2(\mathrm{Ar})(\mathrm{C}=\mathrm{O}) \mathrm{O}\left(\mathrm{CH}_{2}\right)_{2} \mathrm{OCH}_{2} \mathrm{CH}_{2}\right), 69.1(2 \mathrm{C}, 2(\mathrm{Ar})(\mathrm{C}=\mathrm{O})-$ $\left.\mathrm{OCH}_{2} \mathrm{CH}_{2} \mathrm{O}\right), 64.7$ (2C, $\left.2(\mathrm{Ar})(\mathrm{C}=\mathrm{O}) \mathrm{OCH}_{2} \mathrm{CH}_{2} \mathrm{O}\right)$. ESI-MS $(+)$.
Calcd for $\mathrm{C}_{46} \mathrm{H}_{44} \mathrm{Au}_{2} \mathrm{ClO}_{7} \mathrm{P}_{2}^{+}: m / z 1199.1582\left([\mathrm{M}-\mathrm{Cl}]^{+}\right)$. Found: $m / z$ 1199.1633. Calcd for $\mathrm{C}_{46} \mathrm{H}_{44} \mathrm{Au}_{2} \mathrm{Cl}_{2} \mathrm{NaO}_{7} \mathrm{P}_{2}^{+}: m / z$ 1257.1168 ([M $+\mathrm{Na}]^{+}$). Found: $m / z$ 1257.1218.

Compound 5c. According to the general procedure, $\mathrm{AuCl}(\mathrm{tht})$ ( $0.162 \mathrm{~g}, 0.507 \mathrm{mmol}, 2$ equiv), $5 \mathrm{a}(0.206 \mathrm{~g}, 0.253 \mathrm{mmol}, 1$ equiv), and $\mathrm{CH}_{2} \mathrm{Cl}_{2}(35 \mathrm{~mL})$, were stirred for $6 \mathrm{~h}$ at RT. The product was isolated as a white solid. Yield: $0.308 \mathrm{~g}, 0.241 \mathrm{mmol}, 95 \%$. Elem anal. Calcd for $\mathrm{C}_{48} \mathrm{H}_{48} \mathrm{Au}_{2} \mathrm{Cl}_{2} \mathrm{O}_{8} \mathrm{P}_{2}: \mathrm{C}, 45.05 ; \mathrm{H}, 3.78$. Found: $\mathrm{C}, 45.05 ; \mathrm{H}, 3.79 .{ }^{1} \mathrm{H}$ $\mathrm{NMR}\left(\mathrm{CDCl}_{3}\right): \delta_{\mathrm{H}} 8.09-8.12(4 \mathrm{H}, \mathrm{m}, 4 \mathrm{O}(\mathrm{C}=\mathrm{O})(\mathrm{Ar}) \mathrm{CCHCHCP}$, $\left.{ }^{3} J_{\mathrm{H}, \mathrm{H}}=8.6 \mathrm{~Hz},{ }^{4} \mathrm{~J}_{\mathrm{H}, \mathrm{H}}=2.4 \mathrm{~Hz}\right), 7.46-7.60(24 \mathrm{H}, \mathrm{m}, 4 \mathrm{O}(\mathrm{C}=$ O) $(A r) \mathrm{CCHCHCP}, 8 \mathrm{P}(A r) \mathrm{CCHCHCH}, 8 \mathrm{P}(\mathrm{Ar}) \mathrm{CCHCHCH}, 4 \mathrm{P}-$ $(\mathrm{Ar}) \mathrm{CCHCHCH}), 4.47-4.49\left(4 \mathrm{H}, \mathrm{m}, 2 \mathrm{Ar}(\mathrm{C}=\mathrm{O}) \mathrm{OCH}_{2} \mathrm{CH}_{2} \mathrm{O}\right)$, $3.80-3.82\left(4 \mathrm{H}, \mathrm{m}, 2 \mathrm{Ar}(\mathrm{C}=\mathrm{O}) \mathrm{OCH}_{2} \mathrm{CH}_{2} \mathrm{O}\right), 3.66-3.68(4 \mathrm{H}, \mathrm{m}$, $\left.2 \mathrm{Ar}(\mathrm{C}=\mathrm{O}) \mathrm{O}\left(\mathrm{CH}_{2}\right)_{2} \mathrm{OCH}_{2} \mathrm{CH}_{2}\right), 3.62-3.64(4 \mathrm{H}, \mathrm{m}, 2 \mathrm{Ar}(\mathrm{C}=\mathrm{O}) \mathrm{O}-$ $\left.\left(\mathrm{CH}_{2}\right)_{2} \mathrm{OCH}_{2} \mathrm{CH}_{2}\right), 3.63\left(4 \mathrm{H}, \quad \mathrm{s}, \quad 2 \mathrm{Ar}(\mathrm{C}=\mathrm{O}) \mathrm{O}\left(\mathrm{CH}_{2}\right)_{2} \mathrm{O}-\right.$ $\left.\left(\mathrm{CH}_{2}\right)_{2} \mathrm{OCH}_{2}\right) .{ }^{31} \mathrm{P}$ NMR $\left(\mathrm{CDCl}_{3}\right): \delta_{\mathrm{P}} 32.99(2 \mathrm{P}) .{ }^{13} \mathrm{C}$ NMR $\left(\mathrm{CDCl}_{3}\right): \delta_{\mathrm{C}} 165.4(2 \mathrm{C}, 2 \mathrm{O}(\mathrm{C}=\mathrm{O})(\mathrm{Ar}) \mathrm{CCHCHCP}), 134.3(2 \mathrm{C}, \mathrm{d}$, $\left.2 \mathrm{O}(\mathrm{C}=\mathrm{O})(A r) \mathrm{CCHCHCP},{ }^{1} J_{\mathrm{C}, \mathrm{P}}=57 \mathrm{~Hz}\right), 134.3(8 \mathrm{C}, \mathrm{d}, 8 \mathrm{P}(A r)-$ $\left.\mathrm{CCHCHCH},{ }^{2} J_{\mathrm{C}, \mathrm{P}}=14 \mathrm{~Hz}\right), 134.0(4 \mathrm{C}, \mathrm{d}, 4 \mathrm{O}(\mathrm{C}=\mathrm{O})(A r)$ $\left.\mathrm{CCHCHCP},{ }^{2} J_{\mathrm{C}, \mathrm{P}}=14 \mathrm{~Hz}\right), 133.3(2 \mathrm{C}, \mathrm{d}, 2 \mathrm{O}(\mathrm{C}=\mathrm{O})(A r)$ $\left.\mathrm{CCHCHCP},{ }^{4} J_{\mathrm{C}, \mathrm{P}}=2 \mathrm{~Hz}\right), 132.4\left(4 \mathrm{C}, \mathrm{d}, 4 \mathrm{P}(\mathrm{Ar}) \mathrm{CCHCHCH},{ }^{4} \mathrm{~J}_{\mathrm{C}, \mathrm{P}}\right.$ $=3 \mathrm{~Hz}), 130.1\left(4 \mathrm{C}, \mathrm{d}, 4 \mathrm{O}(\mathrm{C}=\mathrm{O})(\mathrm{Ar}) \mathrm{CCHCHCP},{ }^{3} J_{\mathrm{C}, \mathrm{P}}=12 \mathrm{~Hz}\right)$, $129.5\left(8 \mathrm{C}, \mathrm{d}, 8 \mathrm{P}(\mathrm{Ar}) \mathrm{CCHCHCH},{ }^{3} J_{\mathrm{C}, \mathrm{P}}=12 \mathrm{~Hz}\right), 127.9(4 \mathrm{C}, \mathrm{d}$, $\left.4 \mathrm{P}(\mathrm{Ar}) \mathrm{CCHCHCH},{ }^{1} \mathrm{~J}_{\mathrm{C}, \mathrm{P}}=63 \mathrm{~Hz}\right), 70.71,70.66(6 \mathrm{C}, 2(\mathrm{Ar})(\mathrm{C}=$ $\mathrm{O}) \mathrm{O}\left(\mathrm{CH}_{2}\right)_{2} \mathrm{OCH}_{2}, 2(\mathrm{Ar})(\mathrm{C}=\mathrm{O}) \mathrm{O}\left(\mathrm{CH}_{2}\right)_{2} \mathrm{OCH}_{2} \mathrm{CH}_{2}, \quad 2(\mathrm{Ar})(\mathrm{C}=$ O) $\left.\mathrm{O}\left(\mathrm{CH}_{2}\right)_{2} \mathrm{O}\left(\mathrm{CH}_{2}\right)_{2} \mathrm{OCH}_{2}\right), 69.1\left(2 \mathrm{C}, 2(\mathrm{Ar})(\mathrm{C}=\mathrm{O}) \mathrm{OCH}_{2} \mathrm{CH}_{2} \mathrm{O}\right)$, $64.7\left(2 \mathrm{C}, 2(\mathrm{Ar})(\mathrm{C}=\mathrm{O}) \mathrm{OCH}_{2} \mathrm{CH}_{2} \mathrm{O}\right)$. ESI-MS $(+)$. Calcd for $\mathrm{C}_{48} \mathrm{H}_{48} \mathrm{Au}_{2} \mathrm{ClO}_{8} \mathrm{P}_{2}^{+}: \mathrm{m} / z$ 1243.1844 $\left([\mathrm{M}-\mathrm{Cl}]^{+}\right)$. Found: $\mathrm{m} / \mathrm{z}$ 1243.1903. Calcd for $\mathrm{C}_{48} \mathrm{H}_{48} \mathrm{Au}_{2} \mathrm{Cl}_{2} \mathrm{NaO}_{8} \mathrm{P}_{2}^{+}: m / z 1301.1430([\mathrm{M}+$ $\mathrm{Na}]^{+}$). Found: $m / z$ 1301.1469.

Compound $6 c$. According to the general procedure, $\mathrm{AuCl}(\mathrm{tht})$ ( $0.192 \mathrm{~g}, 0.601 \mathrm{mmol}, 2$ equiv), 6a ( $0.257 \mathrm{~g}, 0.299 \mathrm{mmol}, 1$ equiv), and $\mathrm{CH}_{2} \mathrm{Cl}_{2}(35 \mathrm{~mL})$ were stirred $6 \mathrm{~h}$ at $\mathrm{RT}$. The product was isolated as a white solid. Yield: $0.376 \mathrm{~g}, 0.284 \mathrm{mmol}$, 95\%. Elem anal. Calcd for $\mathrm{C}_{50} \mathrm{H}_{52} \mathrm{Au}_{2} \mathrm{Cl}_{2} \mathrm{O}_{9} \mathrm{P}_{2}:$ C, 45.37; H, 3.96. Found: C, 45.44; $\mathrm{H}, 3.84 .{ }^{1} \mathrm{H}$ $\mathrm{NMR}\left(\mathrm{CDCl}_{3}\right): \delta_{\mathrm{H}} 8.08-8.12(4 \mathrm{H}, \mathrm{m}, 4 \mathrm{O}(\mathrm{C}=\mathrm{O})(\mathrm{Ar}) \mathrm{CCHCHCP}$, $\left.{ }^{3} J_{\mathrm{H}, \mathrm{H}}=8.4 \mathrm{~Hz},{ }^{4} J_{\mathrm{H}, \mathrm{H}}=2 \mathrm{~Hz}\right), 7.45-7.59(24 \mathrm{H}, \mathrm{m}, 4 \mathrm{O}(\mathrm{C}=$ O) $(A r) \mathrm{CCHCHCP}, 8 \mathrm{P}(A r) \mathrm{CCHCHCH}, 8 \mathrm{P}(A r) \mathrm{CCHCHCH}, 4 \mathrm{P}-$ $(\mathrm{Ar}) \mathrm{CCHCHCH}), 4.46-4.49\left(4 \mathrm{H}, \mathrm{m}, 2 \mathrm{Ar}(\mathrm{C}=\mathrm{O}) \mathrm{OCH}_{2} \mathrm{CH}_{2} \mathrm{O}\right)$, 3.79-3.82 (4H, m, $\left.2 \mathrm{Ar}(\mathrm{C}=\mathrm{O}) \mathrm{OCH}_{2} \mathrm{CH}_{2} \mathrm{O}\right), 3.65-3.68(4 \mathrm{H}, \mathrm{m}$, $\left.2 \mathrm{Ar}(\mathrm{C}=\mathrm{O}) \mathrm{O}\left(\mathrm{CH}_{2}\right)_{2} \mathrm{OCH}_{2} \mathrm{CH}_{2}\right), 3.60-3.64(4 \mathrm{H}, \mathrm{m}, 2 \mathrm{Ar}(\mathrm{C}=\mathrm{O}) \mathrm{O}-$ $\left.\left(\mathrm{CH}_{2}\right)_{2} \mathrm{OCH}_{2} \mathrm{CH}_{2}\right), 3.59-3.62\left(8 \mathrm{H}, \mathrm{m}, 2 \mathrm{Ar}(\mathrm{C}=\mathrm{O}) \mathrm{O}\left(\mathrm{CH}_{2}\right)_{2} \mathrm{O}-\right.$ $\left(\mathrm{CH}_{2}\right)_{2} \mathrm{O}\left(\mathrm{CH}_{2}\right)_{2} .{ }^{31} \mathrm{P}$ NMR $\left(\mathrm{CDCl}_{3}\right): \delta_{\mathrm{P}} 33.03(2 \mathrm{P}) .{ }^{13} \mathrm{C}$ NMR $\left(\mathrm{CDCl}_{3}\right): \delta_{\mathrm{C}} 165.4(2 \mathrm{C}, 2 \mathrm{O}(\mathrm{C}=\mathrm{O})(\mathrm{Ar}) \mathrm{CCHCHCP}), 134.4(2 \mathrm{C}, \mathrm{d}$, $\left.2 \mathrm{O}(\mathrm{C}=\mathrm{O})(\mathrm{Ar}) \mathrm{CCHCHCP},{ }^{1} J_{\mathrm{C}, \mathrm{P}}=59 \mathrm{~Hz}\right), 134.3(8 \mathrm{C}, \mathrm{d}, 8 \mathrm{P}(A r)-$ $\left.\mathrm{CCHCHCH},{ }^{2} J_{\mathrm{C}, \mathrm{P}}=14 \mathrm{~Hz}\right), 134.1(4 \mathrm{C}, \mathrm{d}, 4 \mathrm{O}(\mathrm{C}=\mathrm{O})(\mathrm{Ar})-$ $\left.\mathrm{CCHCHCP},{ }^{2} J_{\mathrm{C}, \mathrm{P}}=14 \mathrm{~Hz}\right), 133.4(2 \mathrm{C}, 2 \mathrm{O}(\mathrm{C}=\mathrm{O})(\mathrm{Ar}) \mathrm{CCHCHCP})$, $132.5(4 \mathrm{C}, 4 \mathrm{P}(A r) \mathrm{CCHCHCH}), 130.2(4 \mathrm{C}, \mathrm{d}, 4 \mathrm{O}(\mathrm{C}=\mathrm{O})(A r)-$ $\left.\mathrm{CCHCHCP},{ }^{3} J_{\mathrm{C}, \mathrm{P}}=12 \mathrm{~Hz}\right), 129.5\left(8 \mathrm{C}, \mathrm{d}, 8 \mathrm{P}(A r) \mathrm{CCHCHCH},{ }^{3} J_{\mathrm{C}, \mathrm{P}}=\right.$ $12 \mathrm{~Hz}), 128.0\left(4 \mathrm{C}, \mathrm{d}, 4 \mathrm{P}(\mathrm{Ar}) \mathrm{CCHCHCH},{ }^{1} J_{\mathrm{C}, \mathrm{P}}=62 \mathrm{~Hz}\right), 70.78$, $70.74,70.68\left(8 \mathrm{C}, 2(\mathrm{Ar})(\mathrm{C}=\mathrm{O}) \mathrm{O}\left(\mathrm{CH}_{2}\right)_{2} \mathrm{OCH}_{2}, 2(\mathrm{Ar})(\mathrm{C}=\mathrm{O}) \mathrm{O}-\right.$ $\left(\mathrm{CH}_{2}\right)_{2} \mathrm{OCH}_{2} \mathrm{CH}_{2}, 2(\mathrm{Ar})(\mathrm{C}=\mathrm{O}) \mathrm{O}\left(\mathrm{CH}_{2}\right)_{2} \mathrm{O}\left(\mathrm{CH}_{2}\right)_{2} \mathrm{OCH}_{2}, 2(\mathrm{Ar})-$ $\left.(\mathrm{C}=\mathrm{O}) \mathrm{O}\left(\mathrm{CH}_{2}\right)_{2} \mathrm{O}\left(\mathrm{CH}_{2}\right)_{2} \mathrm{OCH}_{2} \mathrm{CH}_{2}\right), 69.2(2 \mathrm{C}, 2(\mathrm{Ar})(\mathrm{C}=\mathrm{O})-$ $\left.\mathrm{OCH}_{2} \mathrm{CH}_{2} \mathrm{O}\right), 64.8\left(2 \mathrm{C}, 2(\mathrm{Ar})(\mathrm{C}=\mathrm{O}) \mathrm{OCH}_{2} \mathrm{CH}_{2} \mathrm{O}\right)$. ESI-MS(+). Calcd for $\mathrm{C}_{50} \mathrm{H}_{52} \mathrm{Au}_{2} \mathrm{ClO}_{9} \mathrm{P}_{2}^{+}: \mathrm{m} / z$ 1287.2106 $\left([\mathrm{M}-\mathrm{Cl}]^{+}\right)$. Found: $m / z$ 1287.2147.Calcd for $\mathrm{C}_{50} \mathrm{H}_{52} \mathrm{Au}_{2} \mathrm{Cl}_{2} \mathrm{NaO}_{9} \mathrm{P}_{2}^{+}: m / z$ 1345.1692 ([M $+\mathrm{Na}]^{+}$). Found: $m / z$ 1345.1719.

Compound 7c. According to the general procedure, $\mathrm{AuCl}(\mathrm{tht})$ $(0.230 \mathrm{~g}, 0.720 \mathrm{mmol}, 2$ equiv), $7 \mathrm{a}(0.340 \mathrm{~g}, 0.359 \mathrm{mmol}, 1$ equiv), and $\mathrm{CH}_{2} \mathrm{Cl}_{2}(35 \mathrm{~mL})$ were stirred for $6 \mathrm{~h}$ at RT. The product was isolated as a white solid. Yield: $0.485 \mathrm{~g}, 0.344 \mathrm{mmol}, 96 \%$. Elem anal. Calcd for $\mathrm{C}_{54} \mathrm{H}_{60} \mathrm{Au}_{2} \mathrm{Cl}_{2} \mathrm{O}_{11} \mathrm{P}_{2}$ : C, 45.94; $\mathrm{H}, 4.28$; Found: $\mathrm{C}, 45.82 ; \mathrm{H}$, 4.04. ${ }^{1} \mathrm{H}$ NMR $\left(\mathrm{CDCl}_{3}\right): \delta_{\mathrm{H}} 8.08-8.12(4 \mathrm{H}, \mathrm{m}, 4 \mathrm{O}(\mathrm{C}=\mathrm{O})(\mathrm{Ar})-$ CCHCHCP, $\left.{ }^{3} \mathrm{~J}_{\mathrm{H}, \mathrm{H}}=8.2 \mathrm{~Hz},{ }^{4} \mathrm{~J}_{\mathrm{H}, \mathrm{H}}=2 \mathrm{~Hz}\right), 7.45-7.59(24 \mathrm{H}, \mathrm{m}$, $4 \mathrm{O}(\mathrm{C}=\mathrm{O})(A r) \mathrm{CCHCHCP}, 8 \mathrm{P}(A r) \mathrm{CCHCHCH}, 8 \mathrm{P}(A r)-$ $\mathrm{CCHCHCH}, 4 \mathrm{P}(\mathrm{Ar}) \mathrm{CCHCHCH}), 4.46-4.49(4 \mathrm{H}, \mathrm{m}, 2 \mathrm{Ar}(\mathrm{C}=$ $\left.\mathrm{O}) \mathrm{OCH}_{2} \mathrm{CH}_{2} \mathrm{O}\right), 3.80-3.82\left(4 \mathrm{H}, \mathrm{m}, 2 \mathrm{Ar}(\mathrm{C}=\mathrm{O}) \mathrm{OCH}_{2} \mathrm{CH}_{2} \mathrm{O}\right)$, 3.66-3.69 (4H, m, $\left.2 \mathrm{Ar}(\mathrm{C}=\mathrm{O}) \mathrm{O}\left(\mathrm{CH}_{2}\right)_{2} \mathrm{OCH}_{2} \mathrm{CH}_{2}\right), 3.61-3.64$ $\left(4 \mathrm{H}, \mathrm{m}, 2 \mathrm{Ar}(\mathrm{C}=\mathrm{O}) \mathrm{O}\left(\mathrm{CH}_{2}\right)_{2} \mathrm{OCH}_{2} \mathrm{CH}_{2}\right), 3.60-3.62(8 \mathrm{H}, \mathrm{m}$, $2 A r(\mathrm{C}=\mathrm{O}) \mathrm{O}\left(\mathrm{CH}_{2}\right)_{2} \mathrm{O}\left(\mathrm{CH}_{2}\right)_{2} \mathrm{O}\left(\mathrm{CH}_{2}\right)_{2}, 3.61(8 \mathrm{H}, \mathrm{s}, 2 \operatorname{Ar}(\mathrm{C}=\mathrm{O}) \mathrm{O}-$ 
$\left(\mathrm{CH}_{2}\right)_{2} \mathrm{O}\left(\mathrm{CH}_{2}\right)_{2} \mathrm{O}\left(\mathrm{CH}_{2}\right)_{2} \mathrm{O}\left(\mathrm{CH}_{2}\right)_{2} .{ }^{31} \mathrm{P}$ NMR $\left(\mathrm{CDCl}_{3}\right): \delta_{\mathrm{P}} 33.01$ $(2 \mathrm{P}) .{ }^{13} \mathrm{C}$ NMR $\left(\mathrm{CDCl}_{3}\right): \delta_{\mathrm{C}} 165.4(2 \mathrm{C}, 2 \mathrm{O}(\mathrm{C}=\mathrm{O})(\mathrm{Ar})-$ CCHCHCP $), 134.4\left(2 \mathrm{C}, \mathrm{d}, 2 \mathrm{O}(\mathrm{C}=\mathrm{O})(\mathrm{Ar}) \mathrm{CCHCHCP},{ }^{1} J_{\mathrm{C}, \mathrm{P}}=58\right.$ $\mathrm{Hz}), 134.3\left(8 \mathrm{C}, \mathrm{d}, 8 \mathrm{P}(\mathrm{Ar}) \mathrm{CCHCHCH},{ }^{2} J_{\mathrm{C}, \mathrm{P}}=14 \mathrm{~Hz}\right), 134.0(4 \mathrm{C}, \mathrm{d}$, $\left.4 \mathrm{O}(\mathrm{C}=\mathrm{O})(\mathrm{Ar}) \mathrm{CCHCHCP},{ }^{2} \mathrm{~J}_{\mathrm{C}, \mathrm{P}}=14 \mathrm{~Hz}\right), 133.4(2 \mathrm{C}, 2 \mathrm{O}(\mathrm{C}=$ O) $\left.(A r) C C H C H C P,{ }^{4} J_{\mathrm{C}, \mathrm{P}}=2 \mathrm{~Hz}\right), 132.4(4 \mathrm{C}, 4 \mathrm{P}(\mathrm{Ar}) \mathrm{CCHCHCH}$, $\left.{ }^{4} \mathrm{~J}_{\mathrm{C}, \mathrm{P}}=2 \mathrm{~Hz}\right), 130.2\left(4 \mathrm{C}, \mathrm{d}, 4 \mathrm{O}(\mathrm{C}=\mathrm{O})(\mathrm{Ar}) \mathrm{CCHCHCP},{ }^{3} \mathrm{~J}_{\mathrm{C}, \mathrm{P}}=12\right.$ $\mathrm{Hz}), 129.5\left(8 \mathrm{C}, \mathrm{d}, 8 \mathrm{P}(\mathrm{Ar}) \mathrm{CCHCHCH},{ }^{3} J_{\mathrm{C}, \mathrm{P}}=12 \mathrm{~Hz}\right), 128.0(4 \mathrm{C}, \mathrm{d}$, $\left.4 \mathrm{P}(\mathrm{Ar}) \mathrm{CCHCHCH},{ }^{1} J_{\mathrm{C}, \mathrm{P}}=63 \mathrm{~Hz}\right), 70.77,70.72,70.66(12 \mathrm{C}$, $2(\mathrm{Ar})(\mathrm{C}=\mathrm{O}) \mathrm{O}\left(\mathrm{CH}_{2}\right)_{2} \mathrm{OCH}_{2}, 2(\mathrm{Ar})(\mathrm{C}=\mathrm{O}) \mathrm{O}\left(\mathrm{CH}_{2}\right)_{2} \mathrm{OCH}_{2} \mathrm{CH}_{2}$, 2$(\mathrm{Ar})(\mathrm{C}=\mathrm{O}) \mathrm{O}\left(\mathrm{CH}_{2}\right)_{2} \mathrm{O}\left(\mathrm{CH}_{2}\right)_{2} \mathrm{OCH}_{2}, 2(\mathrm{Ar})(\mathrm{C}=\mathrm{O}) \mathrm{O}\left(\mathrm{CH}_{2}\right)_{2} \mathrm{O}-$ $\left(\mathrm{CH}_{2}\right)_{2} \mathrm{OCH}_{2} \mathrm{CH}_{2}, 2(\mathrm{Ar})(\mathrm{C}=\mathrm{O}) \mathrm{O}\left(\mathrm{CH}_{2}\right)_{2} \mathrm{O}\left(\mathrm{CH}_{2}\right)_{2} \mathrm{O}\left(\mathrm{CH}_{2}\right)_{2} \mathrm{OCH}_{2}$, $\left.2(\mathrm{Ar})(\mathrm{C}=\mathrm{O}) \mathrm{O}\left(\mathrm{CH}_{2}\right)_{2} \mathrm{O}\left(\mathrm{CH}_{2}\right)_{2} \mathrm{O}\left(\mathrm{CH}_{2}\right)_{2} \mathrm{OCH}_{2} \mathrm{CH}_{2}\right), 69.2(2 \mathrm{C}, 2-$ $\left.(\mathrm{Ar})(\mathrm{C}=\mathrm{O}) \mathrm{OCH}_{2} \mathrm{CH}_{2} \mathrm{O}\right), 64.8 \quad\left(2 \mathrm{C}, 2(\mathrm{Ar})(\mathrm{C}=\mathrm{O}) \mathrm{OCH}_{2} \mathrm{CH}_{2} \mathrm{O}\right)$. ESI-MS(+). Calcd for $\mathrm{C}_{54} \mathrm{H}_{60} \mathrm{Au}_{2} \mathrm{ClO}_{11} \mathrm{P}_{2}^{+}: m / z 1375.2630([\mathrm{M}-$ $\left.\mathrm{Cl}]^{+}\right)$. Found: $m / z$ 1375.2667. Calcd for $\mathrm{C}_{54} \mathrm{H}_{60} \mathrm{Au}_{2} \mathrm{Cl}_{2} \mathrm{NaO}_{11} \mathrm{P}_{2}^{+}: m / z$ $1433.2217\left([\mathrm{M}+\mathrm{Na}]^{+}\right)$. Found: $m / z$ 1433.2239.

Compound $8 \mathrm{c}$. According to the general procedure, $\mathrm{AuCl}(\mathrm{tht})$ (0.318 g, $0.995 \mathrm{mmol}, 1$ equiv), $\mathrm{PPh}_{3}(0.260 \mathrm{~g}, 0.991 \mathrm{mmol}, 1$ equiv), and $\mathrm{CH}_{2} \mathrm{Cl}_{2}(25 \mathrm{~mL})$ were stirred for $4 \mathrm{~h}$ at RT. The product was isolated as a white solid. Yield: $0.471 \mathrm{~g}, 0.952 \mathrm{mmol}, 96 \%$. Elem anal. Calcd for $\mathrm{C}_{18} \mathrm{H}_{15} \mathrm{AuClP}$ : C, 43.70; H, 3.06. Found: C, 44.02; $\mathrm{H}, 2.74$. ${ }^{1} \mathrm{H}$ NMR $\left(\mathrm{CDCl}_{3}\right): \delta_{\mathrm{H}} 7.42-7.58(15 \mathrm{H}, \mathrm{m}, 6 \mathrm{P}(\mathrm{Ar}) \mathrm{CCHCHCH}$, $6 \mathrm{P}(\mathrm{Ar}) \mathrm{CCHCHCH}, 3 \mathrm{P}(\mathrm{Ar}) \mathrm{CCHCHCH}) .{ }^{31} \mathrm{P} \mathrm{NMR}\left(\mathrm{CDCl}_{3}\right): \delta_{\mathrm{P}}$ $33.19(1 \mathrm{P}) .{ }^{13} \mathrm{C} \mathrm{NMR}\left(\mathrm{CDCl}_{3}\right): \delta_{\mathrm{C}} 134.2(6 \mathrm{C}, \mathrm{d}, 6 \mathrm{P}(\mathrm{Ar}) \mathrm{CCHCHCH}$, $\left.{ }^{2} J_{\mathrm{C}, \mathrm{P}}=14 \mathrm{~Hz}\right), 132.1\left(3 \mathrm{C}, \mathrm{d}, 3 \mathrm{P}(\mathrm{Ar}) \mathrm{CCHCHCH},{ }^{4} J_{\mathrm{C}, \mathrm{P}}=3 \mathrm{~Hz}\right), 129.3$ $\left(6 \mathrm{C}, \mathrm{d}, 6 \mathrm{P}(\mathrm{Ar}) \mathrm{CCHCHCH},{ }^{3} J_{\mathrm{C}, \mathrm{P}}=12 \mathrm{~Hz}\right), 128.8(3 \mathrm{C}, \mathrm{d}, 3 \mathrm{P}(\mathrm{Ar})$ $\left.\mathrm{CCHCHCH},{ }^{1} J_{\mathrm{C}, \mathrm{P}}=62 \mathrm{~Hz}\right)$. ESI-MS $(+)$. Calcd for $\mathrm{C}_{18} \mathrm{H}_{15} \mathrm{AuClNaP}^{+}$: $m / z 517.0163\left([\mathrm{M}+\mathrm{Na}]^{+}\right)$. Found: $m / z 517.0163$.

Compound 9c. According to the general procedure, $\mathrm{AuCl}(\mathrm{tht})$ (0.590 g, $1.846 \mathrm{mmol}, 1$ equiv), 4-(diphenylphosphanyl)benzoic acid (0.564 g, $1.841 \mathrm{mmol}, 1$ equiv), and $\mathrm{CH}_{2} \mathrm{Cl}_{2}(25 \mathrm{~mL})$ were stirred for $6 \mathrm{~h}$ at RT. The product was isolated as a white solid. Yield: $0.962 \mathrm{~g}$, $1.786 \mathrm{mmol}, 97 \%$. Elem anal. Calcd for $\mathrm{C}_{19} \mathrm{H}_{15} \mathrm{AuClO}_{2} \mathrm{P}: \mathrm{C}, 42.36$; $\mathrm{H}$, 2.81. Found: $\mathrm{C}, 42.60 ; \mathrm{H}, 2.59 .{ }^{1} \mathrm{H}$ NMR $\left(\mathrm{CDCl}_{3}\right): \delta_{\mathrm{H}} 8.05-8.19(2 \mathrm{H}$, $\left.\mathrm{m}, 2 \mathrm{O}(\mathrm{C}=\mathrm{O})(\mathrm{Ar}) \mathrm{CCHCHCP},{ }^{3} J_{\mathrm{H}, \mathrm{H}}=8.4 \mathrm{~Hz},{ }^{4} \mathrm{~J}_{\mathrm{H}, \mathrm{H}}=2.1 \mathrm{~Hz}\right), 7.47-$ $7.64(12 \mathrm{H}, \quad \mathrm{m}, 2 \mathrm{O}(\mathrm{C}=\mathrm{O})(A r) \mathrm{C} C \mathrm{HCHCP}, 4 \mathrm{P}(A r) \mathrm{CCHCHCH}$, $4 \mathrm{P}(\mathrm{Ar}) \mathrm{CCHCHCH}, 2 \mathrm{P}(\mathrm{Ar}) \mathrm{CCHCHCH}) .{ }^{31} \mathrm{P} \mathrm{NMR}\left(\mathrm{CDCl}_{3}\right): \delta_{\mathrm{P}}$ $33.21(1 \mathrm{P}) .{ }^{13} \mathrm{C}$ NMR $\left(\mathrm{CDCl}_{3}\right): \delta_{\mathrm{C}} 170.5(1 \mathrm{C}, \mathrm{O}(\mathrm{C}=\mathrm{O})(\mathrm{Ar})-$ CCHCHCP $), 135.8\left(1 \mathrm{C}, \mathrm{d}, \mathrm{O}(\mathrm{C}=\mathrm{O})(\mathrm{Ar}) \mathrm{CCHCHCP},{ }_{\mathrm{C}, \mathrm{P}}^{1}=58\right.$ $\mathrm{Hz}), 134.4\left(4 \mathrm{C}, \mathrm{d}, 2 \mathrm{P}(\mathrm{Ar}) \mathrm{CCHCHCH},{ }^{2} J_{\mathrm{C}, \mathrm{P}}=14 \mathrm{~Hz}\right), 134.1(2 \mathrm{C}, \mathrm{d}$, $\left.2 \mathrm{O}(\mathrm{C}=\mathrm{O})(A r) \mathrm{CCHCHCP},{ }^{2} J_{\mathrm{C}, \mathrm{P}}=14 \mathrm{~Hz}\right), 132.4(2 \mathrm{C}, \mathrm{m}, \mathrm{O}(\mathrm{C}=$ O) $(A r) C C H C H C P, 2 P(A r) C C H C H C H), 130.6(2 \mathrm{C}, \mathrm{d}, 2 \mathrm{O}(\mathrm{C}=$ O) $\left.(\mathrm{Ar}) \mathrm{CCHCHCP},{ }^{3} J_{\mathrm{C}, \mathrm{P}}=12 \mathrm{~Hz}\right), 129.6(4 \mathrm{C}, \mathrm{d}, 4 \mathrm{P}(\mathrm{Ar}) \mathrm{CCHCHCH}$, $\left.{ }^{3} J_{\mathrm{C}, \mathrm{P}}=12 \mathrm{~Hz}\right), 128.1\left(2 \mathrm{C}, \mathrm{d}, 2 \mathrm{P}(A r) C \mathrm{CHCHCH},{ }^{1} J_{\mathrm{C}, \mathrm{P}}=61 \mathrm{~Hz}\right)$. Calcd for $\mathrm{C}_{19} \mathrm{H}_{15} \mathrm{AuClO}_{2} \mathrm{P}: m / z$ 538.0164. ESI-MS(-). Found: $m / z$ $537.0193\left([\mathrm{M}-\mathrm{H}]^{-}\right)$. Found: $m / z 1074.8335\left([2 \mathrm{M}-\mathrm{H}]^{-}\right)$.

General Procedure for the Synthesis of the Digold Complexes $1 d-7 d$. The appropriate digold intermediate $1 \mathrm{c}-7 \mathrm{c}$ ( 1 equiv) was added to a suspension of $\beta$-D-thioglucosetetraacetate ( 2 equiv) and $\mathrm{K}_{2} \mathrm{CO}_{3}$ (4 equiv) in degassed acetone under $\mathrm{N}_{2}$. The reaction mixture was stirred under $\mathrm{N}_{2}$ at $\mathrm{RT}$ for $48 \mathrm{~h}$ in the dark, and the reaction evolution was verified by ${ }^{1} \mathrm{H}$ and ${ }^{31} \mathrm{P}$ NMR $\left(\mathrm{CDCl}_{3}\right)$. The reaction mixture was concentrated under reduced pressure, the crude was resuspended in $\mathrm{CH}_{2} \mathrm{Cl}_{2}$ and filtered under vacuum. The filtrate was concentrated under reduced pressure and further dried under high vacuum. The product was isolated as a white solid and stored at -20 ${ }^{\circ} \mathrm{C}$ in the dark.

Compound 1d. According to the general procedure, $\beta$-Dthioglucosetetraacetate $(0.026 \mathrm{~g}, 0.071 \mathrm{mmol}, 2$ equiv $), \mathrm{K}_{2} \mathrm{CO}_{3}$ $(0.050 \mathrm{~g}, 0.362 \mathrm{mmol}, 4$ equiv), and $1 \mathrm{c}(0.100 \mathrm{~g}, 0.091 \mathrm{mmol}, 1$ equiv) in acetone $(25 \mathrm{~mL})$ were stirred for $48 \mathrm{~h}$ at $\mathrm{RT}$ in the dark. The product was isolated as a white solid. Yield: $0.145 \mathrm{~g}, 0.0824 \mathrm{mmol}$, $90 \%$. Elem anal. Calcd for $\mathrm{C}_{68} \mathrm{H}_{70} \mathrm{Au}_{2} \mathrm{O}_{22} \mathrm{P}_{2} \mathrm{~S}_{2}: \mathrm{C}, 46.42 ; \mathrm{H}, 4.01$. Found: C, 46.37; H, 3.92. ${ }^{1} \mathrm{H}$ NMR $\left(\mathrm{CDCl}_{3}\right): \delta_{\mathrm{H}} 8.09-8.12(4 \mathrm{H}, \mathrm{m}$, $\left.4 \mathrm{O}(\mathrm{C}=\mathrm{O})(\mathrm{Ar}) \mathrm{CCHCHCP},{ }^{3} J_{\mathrm{H}, \mathrm{H}}=8.6 \mathrm{~Hz},{ }^{4} J_{\mathrm{H}, \mathrm{H}}=2.0 \mathrm{~Hz}\right), 7.46-$ $7.63(24 \mathrm{H}, \mathrm{m}, 4 \mathrm{O}(\mathrm{C}=\mathrm{O})(A r) \mathrm{CCHCHCP}, 8 \mathrm{P}(A r) \mathrm{CCHCHCH}$, $8 \mathrm{P}(\mathrm{Ar}) \mathrm{CCHCHCH}, 4 \mathrm{P}(\mathrm{Ar}) \mathrm{CCHCHCH}), 5.12-5.17(4 \mathrm{H}, \mathrm{m}$, SCHCHOAc, SCHCHCHOAc), $5.05\left(2 \mathrm{H}, \mathrm{t}, \mathrm{CHCHCH}_{2} \mathrm{OAc},{ }^{3} \mathrm{~J}_{\mathrm{H}, \mathrm{H}}\right.$ $=9.7 \mathrm{~Hz}), 5.03\left(2 \mathrm{H}, \mathrm{t}, \mathrm{SCHCHOAc},{ }^{3} J_{\mathrm{H}, \mathrm{H}}=9.2 \mathrm{~Hz}\right), 4.67(4 \mathrm{H}, \mathrm{s}$,
$\left.\operatorname{Ar}(\mathrm{C}=\mathrm{O}) \mathrm{O}\left(\mathrm{CH}_{2}\right)_{2} \mathrm{O}\right), 4.20\left(2 \mathrm{H}, \mathrm{dd}, \mathrm{CH}_{2} \mathrm{OAc},{ }^{2} \mathrm{~J}_{\mathrm{H}, \mathrm{H}}=12.2 \mathrm{~Hz},{ }^{3} J_{\mathrm{H}, \mathrm{H}}\right.$ $=4.7 \mathrm{~Hz}), 4.09\left(2 \mathrm{H}, \mathrm{dd}, \mathrm{CH}_{2} \mathrm{OAc},{ }^{2} J_{\mathrm{H}, \mathrm{H}}=12.2 \mathrm{~Hz},{ }^{3} J_{\mathrm{H}, \mathrm{H}}=2.3 \mathrm{~Hz}\right)$, $3.75\left(2 \mathrm{H}\right.$, ddd, $\mathrm{CHCH}_{2} \mathrm{OAc},{ }^{3} \mathrm{~J}_{\mathrm{H}, \mathrm{H}}=9.7 \mathrm{~Hz},{ }^{3} \mathrm{~J}_{\mathrm{H}, \mathrm{H}}=4.7 \mathrm{~Hz},{ }^{3} J_{\mathrm{H}, \mathrm{H}}=2.3$ $\mathrm{Hz}), 2.04\left(6 \mathrm{H}, \mathrm{s}, 2 \mathrm{CH}_{2} \mathrm{O}(\mathrm{C}=\mathrm{O}) \mathrm{CH}_{3}\right), 2.00\left(6 \mathrm{H}, \mathrm{s}, 2(\mathrm{C}=\mathrm{O}) \mathrm{CH}_{3}\right)$, $1.96\left(6 \mathrm{H}, \mathrm{s}, 2(\mathrm{C}=\mathrm{O}) \mathrm{CH}_{3}\right), 1.89\left(6 \mathrm{H}, \mathrm{s}, 2(\mathrm{C}=\mathrm{O}) \mathrm{CH}_{3}\right) .{ }^{31} \mathrm{P}$ NMR $\left(\mathrm{CDCl}_{3}\right): \delta_{\mathrm{P}} 38.71(2 \mathrm{P}) \cdot{ }^{13} \mathrm{C}$ NMR $\left(\mathrm{CDCl}_{3}\right): \delta_{\mathrm{C}} 170.8(2 \mathrm{C}, 2(\mathrm{C}=$ O) $\left.\mathrm{CH}_{3}\right), 170.3\left(2 \mathrm{C}, 2(\mathrm{C}=\mathrm{O}) \mathrm{CH}_{3}\right), 169.7\left(2 \mathrm{C}, 2(\mathrm{C}=\mathrm{O}) \mathrm{CH}_{3}\right), 169.6$ (2C, $\left.2(\mathrm{C}=\mathrm{O}) \mathrm{CH}_{3}\right), 165.4(2 \mathrm{C}, 2 \mathrm{O}(\mathrm{C}=\mathrm{O})(\mathrm{Ar}) \mathrm{CCHCHCP}), 135.8$ $\left(2 \mathrm{C}, \mathrm{d}, 2 \mathrm{O}(\mathrm{C}=\mathrm{O})(A r) \mathrm{CCHCHCP},{ }^{1} J_{\mathrm{C}, \mathrm{P}}=53 \mathrm{~Hz}\right), 134.5(4 \mathrm{C}, \mathrm{d}$, $\left.4 \mathrm{P}(A r) \mathrm{CCHCHCH},{ }^{2} J_{\mathrm{C}, \mathrm{P}}=14 \mathrm{~Hz}\right), 134.4(4 \mathrm{C}, \mathrm{d}, 4 \mathrm{P}(\mathrm{Ar}) \mathrm{CCHCHCH}$, $\left.{ }^{2} J_{\mathrm{C}, \mathrm{P}}=14 \mathrm{~Hz}\right), 134.2\left(4 \mathrm{C}, \mathrm{d}, 4 \mathrm{O}(\mathrm{C}=\mathrm{O})(\mathrm{Ar}) \mathrm{CCHCHCP},{ }^{2} J_{\mathrm{C}, \mathrm{P}}=14\right.$ $\mathrm{Hz}), 132.5\left(2 \mathrm{C}, \mathrm{d}, 2 \mathrm{O}(\mathrm{C}=\mathrm{O})(A r) C \mathrm{CHCHCP},{ }^{4} J_{\mathrm{C}, \mathrm{P}}=2 \mathrm{~Hz}\right), 132.1$ $(4 \mathrm{C}, \mathrm{m}, 4 \mathrm{P}(\mathrm{Ar}) \mathrm{CCHCHCH}), 130.1 \quad(4 \mathrm{C}, \mathrm{d}, 4 \mathrm{O}(\mathrm{C}=\mathrm{O})(A r)-$ $\left.\mathrm{CCHCHCP},{ }^{3} J_{\mathrm{C}, \mathrm{P}}=12 \mathrm{~Hz}\right), 129.5\left(8 \mathrm{C}, \mathrm{d}, 8 \mathrm{P}(\mathrm{Ar}) \mathrm{CCHCHCH},{ }^{3} J_{\mathrm{C}, \mathrm{P}}\right.$ $=12 \mathrm{~Hz}), 128.8\left(4 \mathrm{C}, \mathrm{d}, 4 \mathrm{P}(\mathrm{Ar}) \mathrm{CCHCHCH},{ }^{1} J_{\mathrm{C}, \mathrm{P}}=57 \mathrm{~Hz}\right), 83.2(2 \mathrm{C}$, $2 \mathrm{CHCH}_{2} \mathrm{OAc}$ ), 77.8 (2C, SCHCHCHOAc), 75.9 (2C, SCHCHOAc), 74.2 (2C, SCHCHOAc), 69.0 (2C, $\left.2 \mathrm{CHCHCH}_{2} \mathrm{OAc}\right), 63.2$ (2C, $\left.2(\mathrm{Ar})(\mathrm{C}=\mathrm{O}) \mathrm{O}\left(\mathrm{CH}_{2}\right)_{2} \mathrm{O}\right), 62.9\left(2 \mathrm{C}, 2 \mathrm{CHCH}_{2}(\mathrm{C}=\mathrm{O}) \mathrm{CH}_{3}\right), 21.2$ $\left(2 \mathrm{C}, 2 \mathrm{CH}_{2} \mathrm{O}(\mathrm{C}=\mathrm{O}) \mathrm{CH}_{3}\right), 20.82\left(2 \mathrm{C}, 2(\mathrm{C}=\mathrm{O}) \mathrm{CH}_{3}\right), 20.77$ (2C, $\left.2(\mathrm{C}=\mathrm{O}) \mathrm{CH}_{3}\right), 20.72\left(2 \mathrm{C}, 2(\mathrm{C}=\mathrm{O}) \mathrm{CH}_{3}\right)$. ESI-MS $(+)$. Calcd for $\mathrm{C}_{68} \mathrm{H}_{70} \mathrm{Au}_{2} \mathrm{NaO}_{22} \mathrm{P}_{2} \mathrm{~S}_{2}^{+}: m / z 1781.2499\left([\mathrm{M}+\mathrm{Na}]^{+}\right)$. Found: $m / z$ 1781.2609. Calcd for $\mathrm{C}_{54} \mathrm{H}_{51} \mathrm{Au}_{2} \mathrm{O}_{13} \mathrm{P}_{2} \mathrm{~S}^{+}: \mathrm{m} / z$ 1395.1851 ([M $\left.\mathrm{RS}^{-}\right]^{+}$). Found: $m / z$ 1395.2070. UV-vis: $\lambda_{\max }=250 \mathrm{~nm}$.

Compound $2 d$. According to the general procedure, $\beta$-Dthioglucosetetraacetate $(0.107 \mathrm{~g}, 0.294 \mathrm{mmol}, 2$ equiv $), \mathrm{K}_{2} \mathrm{CO}_{3}$ $(0.082 \mathrm{~g}, 0.593 \mathrm{mmol}, 4$ equiv), and $2 \mathrm{c}(0.170 \mathrm{~g}, 0.148 \mathrm{mmol}, 1$ equiv) in acetone $(25 \mathrm{~mL})$ were stirred for $48 \mathrm{~h}$ at RT in the dark. The product was isolated as a white solid. Yield: $0.223 \mathrm{~g}, 0.123 \mathrm{mmol}, 84 \%$. Elem anal. Calcd for $\mathrm{C}_{70} \mathrm{H}_{74} \mathrm{Au}_{2} \mathrm{O}_{23} \mathrm{P}_{2} \mathrm{~S}_{2}$ : C, 46.62; $\mathrm{H}, 4.14$. Found: $\mathrm{C}$, 46.74; $\mathrm{H}, 4.10 .{ }^{1} \mathrm{H}$ NMR $\left(\mathrm{CDCl}_{3}\right): \delta_{\mathrm{H}} 8.08-8.12(4 \mathrm{H}, \mathrm{m}, 4 \mathrm{O}(\mathrm{C}=$ O) $\left.(A r) \mathrm{CCHCHCP},{ }^{3} J_{\mathrm{H}, \mathrm{H}}=8.5 \mathrm{~Hz},{ }^{4} J_{\mathrm{H}, \mathrm{H}}=2 \mathrm{~Hz}\right), 7.47-7.65(24 \mathrm{H}, \mathrm{m}$, $4 \mathrm{O}(\mathrm{C}=\mathrm{O})(A r) \mathrm{CCHCHCP}, 8 \mathrm{P}(A r) \mathrm{CCHCHCH}, 8 \mathrm{P}(A r)-$ $\mathrm{CCHCHCH}, 4 \mathrm{P}(\mathrm{Ar}) \mathrm{CCHCHCH}), 5.13-5.17$ (4H, m, SCHCHOAc, $\mathrm{SCHCHCHOAc}), 5.08\left(2 \mathrm{H}, \mathrm{t}, \mathrm{CHCHCH}_{2} \mathrm{OAc},{ }^{3} \mathrm{~J}_{\mathrm{H} . \mathrm{H}}=9.2 \mathrm{~Hz}\right), 5.04$ $\left(2 \mathrm{H}, \mathrm{t}, \mathrm{SCHCHOAc},{ }^{3} \mathrm{~J}_{\mathrm{H}, \mathrm{H}}=9.2 \mathrm{~Hz}\right), 4.48-4.50(4 \mathrm{H}, \mathrm{m}, 2 \mathrm{Ar}(\mathrm{C}=$ O) $\left.\mathrm{OCH}_{2} \mathrm{CH}_{2} \mathrm{O}\right), 4.21\left(2 \mathrm{H}, \mathrm{dd}, \mathrm{CH}_{2} \mathrm{OAc},{ }^{2} J_{\mathrm{H}, \mathrm{H}}=12.2 \mathrm{~Hz},{ }^{3} J_{\mathrm{H}, \mathrm{H}}=4.8\right.$ $\mathrm{Hz}), 4.09\left(2 \mathrm{H}, \mathrm{dd}, \mathrm{CH}_{2} \mathrm{OAc},{ }^{2} J_{\mathrm{H} H}=12.2 \mathrm{~Hz},{ }^{3} J_{\mathrm{H}}=2.4 \mathrm{~Hz}\right), 3.84-$ $3.87\left(4 \mathrm{H}, \mathrm{m}, 2 \mathrm{Ar}(\mathrm{C}=\mathrm{O}) \mathrm{OCH}_{2} \mathrm{CH}_{2} \mathrm{O}\right), 3.75\left(2 \mathrm{H}, \mathrm{ddd}, \mathrm{CHCH}_{2} \mathrm{OAc}\right.$, $\left.{ }^{3} J_{\mathrm{H}, \mathrm{H}}=9.7 \mathrm{~Hz},{ }^{3} J_{\mathrm{H}, \mathrm{H}}=4.8 \mathrm{~Hz},{ }^{3} J_{\mathrm{H}, \mathrm{H}}=2.4 \mathrm{~Hz}\right), 2.04(6 \mathrm{H}, \mathrm{s}$, $\left.2 \mathrm{CH}_{2} \mathrm{O}(\mathrm{C}=\mathrm{O}) \mathrm{CH}_{3}\right), 2.00\left(6 \mathrm{H}, \mathrm{s}, 2(\mathrm{C}=\mathrm{O}) \mathrm{CH}_{3}\right), 1.96(6 \mathrm{H}, \mathrm{s}$, $\left.2(\mathrm{C}=\mathrm{O}) \mathrm{CH}_{3}\right), 1.89\left(6 \mathrm{H}, \mathrm{s}, 2(\mathrm{C}=\mathrm{O}) \mathrm{CH}_{3}\right) .{ }^{31} \mathrm{P} \mathrm{NMR}\left(\mathrm{CDCl}_{3}\right): \delta_{\mathrm{P}}$ $38.77(2 \mathrm{P}) .{ }^{13} \mathrm{C} \mathrm{NMR}\left(\mathrm{CDCl}_{3}\right): \delta_{\mathrm{C}} 170.9\left(2 \mathrm{C}, 2(\mathrm{C}=\mathrm{O}) \mathrm{CH}_{3}\right), 170.4$ $\left(2 \mathrm{C}, 2(\mathrm{C}=\mathrm{O}) \mathrm{CH}_{3}\right), 169.8\left(2 \mathrm{C}, 2(\mathrm{C}=\mathrm{O}) \mathrm{CH}_{3}\right), 169.7(2 \mathrm{C}, 2(\mathrm{C}=$ O) $\left.\mathrm{CH}_{3}\right), 165.6(2 \mathrm{C}, 2 \mathrm{O}(\mathrm{C}=\mathrm{O})(\mathrm{Ar}) \mathrm{CCHCHCP}), 135.6(2 \mathrm{C}, \mathrm{d}$, $\left.2 \mathrm{O}(\mathrm{C}=\mathrm{O})(A r) \mathrm{CCHCHCP},{ }^{1} J_{\mathrm{C}, \mathrm{P}}=54 \mathrm{~Hz}\right), 134.6(4 \mathrm{C}, \mathrm{d}, 4 \mathrm{P}(A r)-$ $\left.\mathrm{CCHCHCH},{ }^{2} J_{\mathrm{Cp}}=14 \mathrm{~Hz}\right), 134.5\left(4 \mathrm{C}, \mathrm{d}, 4 \mathrm{P}(\mathrm{Ar}) \mathrm{CCHCHCH},{ }^{2} J_{\mathrm{CP}}=\right.$ $14 \mathrm{~Hz}), 134.2\left(4 \mathrm{C}, \mathrm{d}, 4 \mathrm{O}(\mathrm{C}=\mathrm{O})(\mathrm{Ar}) \mathrm{CCHCHCP},{ }^{2} J_{\mathrm{C}, \mathrm{P}}=14 \mathrm{~Hz}\right)$, $132.9\left(2 \mathrm{C}, \mathrm{d}, 2 \mathrm{O}(\mathrm{C}=\mathrm{O})(A r) C \mathrm{CHCHCP},{ }^{4} J_{\mathrm{C}, \mathrm{P}}=2 \mathrm{~Hz}\right), 132.1(4 \mathrm{C}, \mathrm{d}$, $\left.4 \mathrm{P}(\mathrm{Ar}) \mathrm{CCHCHCH},{ }^{4} \mathrm{~J}_{\mathrm{C}, \mathrm{P}}=2 \mathrm{~Hz}\right), 130.1(4 \mathrm{C}, \mathrm{d}, 4 \mathrm{O}(\mathrm{C}=\mathrm{O})(A r)-$ $\left.\mathrm{CCHCHCP},{ }^{3} J_{\mathrm{C}, \mathrm{P}}=12 \mathrm{~Hz}\right), 129.5\left(8 \mathrm{C}, \mathrm{d}, 8 \mathrm{P}(\mathrm{Ar}) \mathrm{CCHCHCH},{ }^{3} J_{\mathrm{C}, \mathrm{P}}=\right.$ $12 \mathrm{~Hz}), 129.0\left(4 \mathrm{C}, \mathrm{d}, 4 \mathrm{P}(\mathrm{Ar}) \mathrm{CCHCHCH},{ }^{1} J_{\mathrm{C}, \mathrm{P}}=57 \mathrm{~Hz}\right), 83.3(2 \mathrm{C}$, $2 \mathrm{CHCH}_{2} \mathrm{OAc}$ ), 77.8 (2C, SCHCHCHOAc), 75.9 (2C, SCHCHOAc), 74.3 (2C, SCHCHOAc), 69.2 (2C, $\left.2 \mathrm{CHCHCH}_{2} \mathrm{OAc}\right), 69.1$ (2C, $\left.2(\mathrm{Ar})(\mathrm{C}=\mathrm{O}) \mathrm{OCH}_{2} \mathrm{CH}_{2} \mathrm{O}\right), 64.6\left(2 \mathrm{C}, 2(\mathrm{Ar})(\mathrm{C}=\mathrm{O}) \mathrm{OCH}_{2} \mathrm{CH}_{2} \mathrm{O}\right)$, $63.0\left(2 \mathrm{C}, 2 \mathrm{CHCH}_{2}(\mathrm{C}=\mathrm{O}) \mathrm{CH}_{3}\right), 21.3\left(2 \mathrm{C}, 2 \mathrm{CH}_{2} \mathrm{O}(\mathrm{C}=\mathrm{O}) \mathrm{CH}_{3}\right)$, $20.86\left(2 \mathrm{C}, 2(\mathrm{C}=\mathrm{O}) \mathrm{CH}_{3}\right), 20.82\left(2 \mathrm{C}, 2(\mathrm{C}=\mathrm{O}) \mathrm{CH}_{3}\right), 20.77(2 \mathrm{C}$, $\left.2(\mathrm{C}=\mathrm{O}) \mathrm{CH}_{3}\right)$. ESI-MS $(+)$. Calcd for $\mathrm{C}_{70} \mathrm{H}_{74} \mathrm{Au}_{2} \mathrm{NaO}_{23} \mathrm{P}_{2} \mathrm{~S}_{2}{ }^{+}: \mathrm{m} / z$ $1825.2761\left([\mathrm{M}+\mathrm{Na}]^{+}\right)$. Found: $m / z$ 1825.2839. Calcd for $\mathrm{C}_{56} \mathrm{H}_{55} \mathrm{Au}_{2} \mathrm{O}_{14} \mathrm{P}_{2} \mathrm{~S}^{+}: \mathrm{m} / z 1439.2113\left(\left[\mathrm{M}-\mathrm{RS}^{-}\right]^{+}\right)$. Found: $\mathrm{m} / z$ 1439.2242. UV-vis: $\lambda_{\max }=250 \mathrm{~nm}$.

Compound $3 d$. According to the general procedure, $\beta$-Dthioglucosetetraacetate $(0.107 \mathrm{~g}, 0.294 \mathrm{mmol}, 2$ equiv $), \mathrm{K}_{2} \mathrm{CO}_{3}$ (0.081 g, $0.586 \mathrm{mmol}, 4$ equiv), and $3 \mathrm{c}(0.175 \mathrm{~g}, 0.147 \mathrm{mmol}, 1$ equiv) in acetone $(25 \mathrm{~mL})$ were stirred for $48 \mathrm{~h}$ at $\mathrm{RT}$ in the dark. The product was isolated as a white solid. Yield: $0.258 \mathrm{~g}, 0.140 \mathrm{mmol}, 95 \%$. Elem anal. Calcd for $\mathrm{C}_{72} \mathrm{H}_{78} \mathrm{Au}_{2} \mathrm{O}_{24} \mathrm{P}_{2} \mathrm{~S}_{2}: \mathrm{C}, 46.81 ; \mathrm{H}, 4.26$. Found: $\mathrm{C}$, 47.02; $\mathrm{H}, 3.98 .{ }^{1} \mathrm{H}$ NMR $\left(\mathrm{CDCl}_{3}\right): \delta_{\mathrm{H}} 8.09-8.12(4 \mathrm{H}, \mathrm{m}, 4 \mathrm{O}(\mathrm{C}=$ O) $\left.(A r) \mathrm{CCHCHCP},{ }^{3} J_{\mathrm{H}, \mathrm{H}}=8.5 \mathrm{~Hz},{ }^{4} J_{\mathrm{H}, \mathrm{H}}=2.0 \mathrm{~Hz}\right), 7.47-7.67(24 \mathrm{H}$, $\mathrm{m}, \quad 4 \mathrm{O}(\mathrm{C}=\mathrm{O})(A r) \mathrm{CCHCHCP}, 8 \mathrm{P}(A r) \mathrm{CCHCHCH}, 8 \mathrm{P}(A r)-$ $\mathrm{CCHCHCH}, 4 \mathrm{P}(\mathrm{Ar}) \mathrm{CCHCHCH}), 5.11-5.18(4 \mathrm{H}, \mathrm{m}, \mathrm{SCHCHOAc}$, 
SCHCHCHOAc), $5.07\left(2 \mathrm{H}, \mathrm{t}, \mathrm{CHCHCH}_{2} \mathrm{OAc},{ }^{3} \mathrm{~J}_{\mathrm{H} . \mathrm{H}}=9.1 \mathrm{~Hz}\right), 5.04$ $\left(2 \mathrm{H}, \mathrm{t}, \mathrm{SCHCHOAc},{ }^{3} J_{\mathrm{H}, \mathrm{H}}=9.2 \mathrm{~Hz}\right), 4.46-4.48(4 \mathrm{H}, \mathrm{m}, 2 \operatorname{Ar}(\mathrm{C}=$ O) $\left.\mathrm{OCH}_{2} \mathrm{CH}_{2} \mathrm{O}\right), 4.22\left(2 \mathrm{H}, \mathrm{dd}, \mathrm{CH}_{2} \mathrm{OAc},{ }^{2} \mathrm{~J}_{\mathrm{H}, \mathrm{H}}=12.2 \mathrm{~Hz},{ }^{3} J_{\mathrm{H}, \mathrm{H}}=4.8\right.$ $\mathrm{Hz}), 4.10\left(2 \mathrm{H}, \mathrm{dd}, \mathrm{CH}_{2} \mathrm{OAc},{ }^{2} J_{\mathrm{H}, \mathrm{H}}=12.2 \mathrm{~Hz},{ }^{3} J_{\mathrm{H}, \mathrm{H}}=2.4 \mathrm{~Hz}\right), 3.80-$ $3.83\left(4 \mathrm{H}, \mathrm{m}, 2 \mathrm{Ar}(\mathrm{C}=\mathrm{O}) \mathrm{OCH}_{2} \mathrm{CH}_{2} \mathrm{O}\right), 3.76\left(2 \mathrm{H}, \mathrm{ddd}, \mathrm{CHCH}_{2} \mathrm{OAc}\right.$, $\left.{ }^{3} J_{\mathrm{H}, \mathrm{H}}=9.8 \mathrm{~Hz},{ }^{3} J_{\mathrm{H}, \mathrm{H}}=4.8 \mathrm{~Hz},{ }^{3} J_{\mathrm{H}, \mathrm{H}}=2.4 \mathrm{~Hz}\right), 3.69(4 \mathrm{H}, \mathrm{s}, 2 \operatorname{Ar}(\mathrm{C}=$ O) $\left.\mathrm{O}\left(\mathrm{CH}_{2}\right)_{2} \mathrm{OCH}_{2}\right), 2.05\left(6 \mathrm{H}, \mathrm{s}, 2 \mathrm{CH}_{2} \mathrm{O}(\mathrm{C}=\mathrm{O}) \mathrm{CH}_{3}\right), 2.01(6 \mathrm{H}, \mathrm{s}$, $\left.2(\mathrm{C}=\mathrm{O}) \mathrm{CH}_{3}\right), 1.97\left(6 \mathrm{H}, \mathrm{s}, 2(\mathrm{C}=\mathrm{O}) \mathrm{CH}_{3}\right), 1.90(6 \mathrm{H}, \mathrm{s}, 2(\mathrm{C}=$ O) $\left.\mathrm{CH}_{3}\right) .{ }^{31} \mathrm{P}$ NMR $\left(\mathrm{CDCl}_{3}\right): \delta_{\mathrm{P}} 38.79(2 \mathrm{P}) .{ }^{13} \mathrm{C} \mathrm{NMR}\left(\mathrm{CDCl}_{3}\right): \delta_{\mathrm{C}}$ $170.9\left(2 \mathrm{C}, 2(\mathrm{C}=\mathrm{O}) \mathrm{CH}_{3}\right), 170.4\left(2 \mathrm{C}, 2(\mathrm{C}=\mathrm{O}) \mathrm{CH}_{3}\right), 169.8(2 \mathrm{C}$, $\left.2(\mathrm{C}=\mathrm{O}) \mathrm{CH}_{3}\right), 169.7 \quad\left(2 \mathrm{C}, 2(\mathrm{C}=\mathrm{O}) \mathrm{CH}_{3}\right), 165.6(2 \mathrm{C}, 2 \mathrm{O}(\mathrm{C}=$ $\mathrm{O})(\mathrm{Ar}) \mathrm{CCHCHCP}), 135.6\left(2 \mathrm{C}, \mathrm{d}, 2 \mathrm{O}(\mathrm{C}=\mathrm{O})(\mathrm{Ar}) \mathrm{CCHCHCP},{ }^{1} J_{\mathrm{C}, \mathrm{P}}\right.$ $=53 \mathrm{~Hz}), 134.6\left(4 \mathrm{C}, \mathrm{d}, 4 \mathrm{P}(\mathrm{Ar}) \mathrm{CCHCHCH},{ }^{2} \mathrm{~J}_{\mathrm{C}, \mathrm{P}}=14 \mathrm{~Hz}\right), 134.5$ $\left(4 \mathrm{C}, \mathrm{d}, 4 \mathrm{P}(\mathrm{Ar}) \mathrm{CCHCHCH},{ }^{2} J_{\mathrm{C}, \mathrm{P}}=14 \mathrm{~Hz}\right), 134.2(4 \mathrm{C}, \mathrm{d}, 4 \mathrm{O}(\mathrm{C}=$ $\left.\mathrm{O})(\mathrm{Ar}) \mathrm{CCHCHCP},{ }^{2} \mathrm{~J}_{\mathrm{C}, \mathrm{P}}=14 \mathrm{~Hz}\right), 133.0(2 \mathrm{C}, \mathrm{d}, 2 \mathrm{O}(\mathrm{C}=\mathrm{O})(\mathrm{Ar})$ CCHCHCP, $\left.{ }^{4} J_{\mathrm{C}, \mathrm{P}}=2 \mathrm{~Hz}\right), 132.1(4 \mathrm{C}, \mathrm{m}, 4 \mathrm{P}(\mathrm{Ar}) \mathrm{CCHCHCH}), 130.1$ $\left(4 \mathrm{C}, \mathrm{d}, 4 \mathrm{O}(\mathrm{C}=\mathrm{O})(\mathrm{Ar}) \mathrm{CCHCHCP},{ }^{3} \mathrm{C}_{\mathrm{C}, \mathrm{P}}=12 \mathrm{~Hz}\right), 129.5(8 \mathrm{C}, \mathrm{d}$, $\left.8 \mathrm{P}(\mathrm{Ar}) \mathrm{CCHCHCH},{ }^{3} \mathrm{~J}_{\mathrm{C}, \mathrm{P}}=12 \mathrm{~Hz}\right), 129.0(4 \mathrm{C}, \mathrm{d}, 4 \mathrm{P}(\mathrm{Ar}) \mathrm{CCHCHCH}$, $\left.{ }^{1} J_{\mathrm{C}, \mathrm{P}}=57 \mathrm{~Hz}\right), 83.3\left(2 \mathrm{C}, 2 \mathrm{CHCH}_{2} \mathrm{OAc}\right), 77.8$ (2C, SCHCHCHOAc), 75.9 (2C, SCHCHOAc), 74.3 (2C, SCHCHOAc), 70.8 (2C, $\left.2(A r)(\mathrm{C}=\mathrm{O}) \mathrm{O}\left(\mathrm{CH}_{2}\right)_{2} \mathrm{OCH}_{2}\right), 69.3(2 \mathrm{C}, \quad 2(A r)(\mathrm{C}=\mathrm{O})-$ $\left.\mathrm{OCH}_{2} \mathrm{CH}_{2} \mathrm{O}\right), 69.1\left(2 \mathrm{C}, 2 \mathrm{CHCHCH}_{2} \mathrm{OAc}\right), 64.6(2 \mathrm{C}, 2(\mathrm{Ar})(\mathrm{C}=$ $\left.\mathrm{O}) \mathrm{OCH}_{2} \mathrm{CH}_{2} \mathrm{O}\right), 63.0\left(2 \mathrm{C}, 2 \mathrm{CHCH}_{2}(\mathrm{C}=\mathrm{O}) \mathrm{CH}_{3}\right), 21.3(2 \mathrm{C}$, $\left.2 \mathrm{CH}_{2} \mathrm{O}(\mathrm{C}=\mathrm{O}) \mathrm{CH}_{3}\right), 20.87\left(2 \mathrm{C}, 2(\mathrm{C}=\mathrm{O}) \mathrm{CH}_{3}\right), 20.84(2 \mathrm{C}, 2(\mathrm{C}=$ O) $\left.\mathrm{CH}_{3}\right), 20.79\left(2 \mathrm{C}, 2(\mathrm{C}=\mathrm{O}) \mathrm{CH}_{3}\right)$. ESI-MS $(+)$. Calcd for $\mathrm{C}_{72} \mathrm{H}_{78} \mathrm{Au}_{2} \mathrm{NaO}_{24} \mathrm{P}_{2} \mathrm{~S}_{2}^{+}: m / z$ 1869.3023 $\left([\mathrm{M}+\mathrm{Na}]^{+}\right)$. Found: $m / z$ 1869.3127. Calcd for $\mathrm{C}_{58} \mathrm{H}_{59} \mathrm{Au}_{2} \mathrm{O}_{15} \mathrm{P}_{2} \mathrm{~S}^{+}: m / z$ 1483.2375 ([M $\left.\mathrm{RS}^{-}\right]^{+}$). Found: $m / z$ 1483.2581. UV-vis: $\lambda_{\max }=250 \mathrm{~nm}$.

Compound 4d. According to the general procedure, $\beta$-Dthioglucosetetraacetate $(0.103 \mathrm{~g}, 0.283 \mathrm{mmol}, 2$ equiv $), \mathrm{K}_{2} \mathrm{CO}_{3}$ $(0.079 \mathrm{~g}, 0.572 \mathrm{mmol}, 4$ equiv), and $4 \mathrm{c}(0.175 \mathrm{~g}, 1$ equiv) in acetone $(25 \mathrm{~mL})$ were stirred for $48 \mathrm{~h}$ at $\mathrm{RT}$ in the dark. The product was isolated as a white solid. Yield: $0.233 \mathrm{~g}, 0.123 \mathrm{mmol}, 87 \%$. Elem anal. Calcd for $\mathrm{C}_{74} \mathrm{H}_{82} \mathrm{Au}_{2} \mathrm{O}_{25} \mathrm{P}_{2} \mathrm{~S}_{2}$ : C, 46.99; $\mathrm{H}, 4.37$. Found: C, 47.02; $\mathrm{H}$, 4.29. ${ }^{1} \mathrm{H}$ NMR $\left(\mathrm{CDCl}_{3}\right): \delta_{\mathrm{H}} 8.09-8.13(4 \mathrm{H}, \mathrm{m}, 4 \mathrm{O}(\mathrm{C}=\mathrm{O})(A r)-$ $\left.\mathrm{CCHCHCP},{ }^{3} J_{\mathrm{H}, \mathrm{H}}=8.5 \mathrm{~Hz},{ }^{4} J_{\mathrm{H}, \mathrm{H}}=2.0 \mathrm{~Hz}\right), 7.46-7.64(24 \mathrm{H}, \mathrm{m}$, $4 \mathrm{O}(\mathrm{C}=\mathrm{O})(A r) \mathrm{CCHCHCP}, 8 \mathrm{P}(A r) \mathrm{CCHCHCH}, 8 \mathrm{P}(A r)-$ $\mathrm{CCHCHCH}, 4 \mathrm{P}(A r) \mathrm{CCHCHCH}), 5.13-5.18(4 \mathrm{H}, \mathrm{m}, \mathrm{SCHCHOAc}$, $\mathrm{SCHCHCHOAc}), 5.09\left(2 \mathrm{H}, \mathrm{t}, \mathrm{CHCHCH}_{2} \mathrm{OAc},{ }^{3} \mathrm{~J}_{\mathrm{H}, \mathrm{H}}=9.6 \mathrm{~Hz}\right), 5.04$ $\left(2 \mathrm{H}, \mathrm{t}, \mathrm{SCHCHOAc},{ }^{3} J_{\mathrm{H}, \mathrm{H}}=9.2 \mathrm{~Hz}\right), 4.46-4.48(4 \mathrm{H}, \mathrm{m}, 2 \mathrm{Ar}(\mathrm{C}=$ O) $\left.\mathrm{OCH}_{2} \mathrm{CH}_{2} \mathrm{O}\right), 4.21\left(2 \mathrm{H}\right.$, dd, $\mathrm{CH}_{2} \mathrm{OAc},{ }^{2} J_{\mathrm{H}, \mathrm{H}}=12.3 \mathrm{~Hz},{ }^{3} J_{\mathrm{H}, \mathrm{H}}=4.8$ $\mathrm{Hz}), 4.10\left(2 \mathrm{H}, \mathrm{dd}, \mathrm{CH}_{2} \mathrm{OAc},{ }^{2} \mathrm{~J}_{\mathrm{H}, \mathrm{H}}=12.3 \mathrm{~Hz},{ }^{3} \mathrm{~J}_{\mathrm{H}, \mathrm{H}}=2.3 \mathrm{~Hz}\right), 3.79-$ $3.81\left(4 \mathrm{H}, \mathrm{m}, 2 \mathrm{Ar}(\mathrm{C}=\mathrm{O}) \mathrm{OCH}_{2} \mathrm{CH}_{2} \mathrm{O}\right), 3.75\left(2 \mathrm{H}, \mathrm{ddd}, \mathrm{CHCH}_{2} \mathrm{OAc}\right.$, $\left.{ }^{3} J_{\mathrm{H}, \mathrm{H}}=9.8 \mathrm{~Hz},{ }^{3} J_{\mathrm{H}, \mathrm{H}}=4.8 \mathrm{~Hz},{ }^{3} J_{\mathrm{H}, \mathrm{H}}=2.3 \mathrm{~Hz}\right), 3.63-3.70(8 \mathrm{H}, \mathrm{m}$, $\left.2 \mathrm{Ar}(\mathrm{C}=\mathrm{O}) \mathrm{O}\left(\mathrm{CH}_{2}\right)_{2} \mathrm{O}\left(\mathrm{CH}_{2}\right)_{2}\right), 2.04\left(6 \mathrm{H}, \mathrm{s}, 2 \mathrm{CH}_{2} \mathrm{O}(\mathrm{C}=\mathrm{O}) \mathrm{CH}_{3}\right)$, $2.01\left(6 \mathrm{H}, \mathrm{s}, 2(\mathrm{C}=\mathrm{O}) \mathrm{CH}_{3}\right), 1.97\left(6 \mathrm{H}, \mathrm{s}, 2(\mathrm{C}=\mathrm{O}) \mathrm{CH}_{3}\right), 1.90(6 \mathrm{H}, \mathrm{s}$, $\left.2(\mathrm{C}=\mathrm{O}) \mathrm{CH}_{3}\right) .{ }^{31} \mathrm{P}$ NMR $\left(\mathrm{CDCl}_{3}\right): \delta_{\mathrm{P}} 38.71(2 \mathrm{P}) .{ }^{13} \mathrm{C}$ NMR $\left(\mathrm{CDCl}_{3}\right): \delta_{\mathrm{C}} 170.9\left(2 \mathrm{C}, 2(\mathrm{C}=\mathrm{O}) \mathrm{CH}_{3}\right), 170.4\left(2 \mathrm{C}, 2(\mathrm{C}=\mathrm{O}) \mathrm{CH}_{3}\right)$, $169.8\left(2 \mathrm{C}, 2(\mathrm{C}=\mathrm{O}) \mathrm{CH}_{3}\right), 169.7\left(2 \mathrm{C}, 2(\mathrm{C}=\mathrm{O}) \mathrm{CH}_{3}\right), 165.6(2 \mathrm{C}$, $2 \mathrm{O}(\mathrm{C}=\mathrm{O})(A r) \mathrm{CCHCHCP}), 135.5 \quad(2 \mathrm{C}, \mathrm{d}, 2 \mathrm{O}(\mathrm{C}=\mathrm{O})(A r)-$ $\left.\mathrm{CCHCHCP},{ }^{1} J_{\mathrm{C}, \mathrm{P}}=54 \mathrm{~Hz}\right), 134.5\left(4 \mathrm{C}, \mathrm{d}, 4 \mathrm{P}(\mathrm{Ar}) \mathrm{CCHCHCH},{ }^{2} J_{\mathrm{C}, \mathrm{P}}\right.$ $=14 \mathrm{~Hz}), 134.4\left(4 \mathrm{C}, \mathrm{d}, 4 \mathrm{P}(\mathrm{Ar}) \mathrm{CCHCHCH},{ }^{2} J_{\mathrm{C}, \mathrm{P}}=14 \mathrm{~Hz}\right), 134.1$ $\left(4 \mathrm{C}, \mathrm{d}, 4 \mathrm{O}(\mathrm{C}=\mathrm{O})(\mathrm{Ar}) \mathrm{CCHCHCP},{ }^{2} \mathrm{~J}_{\mathrm{C}, \mathrm{P}}=14 \mathrm{~Hz}\right), 133.0(2 \mathrm{C}, \mathrm{d}$, $\left.2 \mathrm{O}(\mathrm{C}=\mathrm{O})(A r) C \mathrm{CHCHCP},{ }^{4} J_{\mathrm{C}, \mathrm{P}}=2 \mathrm{~Hz}\right), 132.1(4 \mathrm{C}, \mathrm{m}, 4 \mathrm{P}(A r)-$ $\mathrm{CCHCHCH}), 130.1\left(4 \mathrm{C}, \mathrm{d}, 4 \mathrm{O}(\mathrm{C}=\mathrm{O})(\mathrm{Ar}) \mathrm{CCHCHCP},{ }^{3} \mathrm{~J}_{\mathrm{P}, \mathrm{P}}=12\right.$ $\mathrm{Hz}), 129.5\left(8 \mathrm{C}, \mathrm{d}, 8 \mathrm{P}(\mathrm{Ar}) \mathrm{CCHCHCH},{ }^{3} J_{\mathrm{C}, \mathrm{P}}=12 \mathrm{~Hz}\right), 129.0(4 \mathrm{C}, \mathrm{d}$, $\left.4 \mathrm{P}(\mathrm{Ar}) \mathrm{CCHCHCH},{ }^{1} \mathrm{~J}_{\mathrm{C}, \mathrm{P}}=57 \mathrm{~Hz}\right), 83.3\left(2 \mathrm{C}, 2 \mathrm{CHCH}_{2} \mathrm{OAc}\right), 77.8$ (2C, SCHCHCHOAc), 75.9 (2C, SCHCHOAc), 74.3 (2C, SCHCHOAc), $70.80,70.78\left(4 \mathrm{C}, 2(\mathrm{Ar})(\mathrm{C}=\mathrm{O}) \mathrm{O}\left(\mathrm{CH}_{2}\right)_{2} \mathrm{OCH}_{2}\right.$, $\left.2(\mathrm{Ar})(\mathrm{C}=\mathrm{O}) \mathrm{O}\left(\mathrm{CH}_{2}\right)_{2} \mathrm{OCH}_{2} \mathrm{CH}_{2}\right), 69.2(2 \mathrm{C}, 2(\mathrm{Ar})(\mathrm{C}=\mathrm{O})-$ $\left.\mathrm{OCH}_{2} \mathrm{CH}_{2} \mathrm{O}\right), 69.1\left(2 \mathrm{C}, 2 \mathrm{CHCHCH}_{2} \mathrm{OAc}\right), 64.7(2 \mathrm{C}, 2(\mathrm{Ar})(\mathrm{C}=$ O) $\left.\mathrm{OCH}_{2} \mathrm{CH}_{2} \mathrm{O}\right), 63.0\left(2 \mathrm{C}, 2 \mathrm{CHCH}_{2}(\mathrm{C}=\mathrm{O}) \mathrm{CH}_{3}\right), 21.3(2 \mathrm{C}$, $\left.2 \mathrm{CH}_{2} \mathrm{O}(\mathrm{C}=\mathrm{O}) \mathrm{CH}_{3}\right), 20.86\left(2 \mathrm{C}, 2(\mathrm{C}=\mathrm{O}) \mathrm{CH}_{3}\right), 20.82(2 \mathrm{C}, 2(\mathrm{C}=$ O) $\left.\mathrm{CH}_{3}\right), 20.77\left(2 \mathrm{C}, 2(\mathrm{C}=\mathrm{O}) \mathrm{CH}_{3}\right)$. ESI-MS(+). Calcd for $\mathrm{C}_{74} \mathrm{H}_{82} \mathrm{Au}_{2} \mathrm{NaO}_{25} \mathrm{P}_{2} \mathrm{~S}_{2}^{+}: m / z$ 1913.3285 $\left([\mathrm{M}+\mathrm{Na}]^{+}\right)$. Found: $m / z$ 1913.3390. Calcd for $\mathrm{C}_{60} \mathrm{H}_{63} \mathrm{Au}_{2} \mathrm{O}_{16} \mathrm{P}_{2} \mathrm{~S}^{+}: \mathrm{m} / z$ 1527.2638 ([M $\left.\mathrm{RS}^{-}\right]^{+}$). Found: $m / z$ 1527.2816. UV-vis: $\lambda_{\max }=250 \mathrm{~nm}$.

Compound $5 \boldsymbol{d}$. According to the general procedure, $\beta$-Dthioglucosetetraacetate $(0.100 \mathrm{~g}, 0.274 \mathrm{mmol}, 2$ equiv $), \mathrm{K}_{2} \mathrm{CO}_{3}$ $(0.076 \mathrm{~g}, 0.550 \mathrm{mmol}, 4$ equiv), and $5 \mathrm{c}(0.175 \mathrm{~g}, 0.137 \mathrm{mmol}, 1$ equiv) in acetone $(25 \mathrm{~mL})$ were stirred for $48 \mathrm{~h}$ at $\mathrm{RT}$ in the dark. The product was isolated as a white solid. Yield: $0.253 \mathrm{~g}, 0.131 \mathrm{mmol}, 96 \%$. Elem anal. Calcd for $\mathrm{C}_{76} \mathrm{H}_{86} \mathrm{Au}_{2} \mathrm{O}_{26} \mathrm{P}_{2} \mathrm{~S}_{2}$ : $\mathrm{C}, 47.16 ; \mathrm{H}, 4.48$. Found: $\mathrm{C}$, 47.30; $\mathrm{H}, 4.20 .{ }^{1} \mathrm{H}$ NMR $\left(\mathrm{CDCl}_{3}\right): \delta_{\mathrm{H}} 8.09-8.13(4 \mathrm{H}, \mathrm{m}, 4 \mathrm{O}(\mathrm{C}=$ O) $\left.(\mathrm{Ar}) \mathrm{CCHCHCP},{ }^{3} \mathrm{~J}_{\mathrm{H}, \mathrm{H}}=8.5 \mathrm{~Hz},{ }^{4} \mathrm{~J}_{\mathrm{H}, \mathrm{H}}=2.0 \mathrm{~Hz}\right), 7.46-7.64(24 \mathrm{H}$, $\mathrm{m}, \quad 4 \mathrm{O}(\mathrm{C}=\mathrm{O})(A r) \mathrm{CCHCHCP}, 8 \mathrm{P}(A r) \mathrm{CCHCHCH}, 8 \mathrm{P}(A r)$ $\mathrm{CCHCHCH}, 4 \mathrm{P}(\mathrm{Ar}) \mathrm{CCHCHCH}), 5.11-5.17(4 \mathrm{H}, \mathrm{m}, \mathrm{SCHCHOAc}$, $\mathrm{SCHCHCHOAc}), 5.08\left(2 \mathrm{H}, \mathrm{t}, \mathrm{CHCHCH}_{2} \mathrm{OAc},{ }^{3} \mathrm{~J}_{\mathrm{H}, \mathrm{H}}=9.6 \mathrm{~Hz}\right), 5.04$ $\left(2 \mathrm{H}, \mathrm{t}, \mathrm{SCHCHOAc},{ }^{3} \mathrm{~J}_{\mathrm{H}, \mathrm{H}}=9.2 \mathrm{~Hz}\right), 4.46-4.48(4 \mathrm{H}, \mathrm{m}, 2 \mathrm{Ar}(\mathrm{C}=$ O) $\left.\mathrm{OCH}_{2} \mathrm{CH}_{2} \mathrm{O}\right), 4.21\left(2 \mathrm{H}\right.$, dd, $\mathrm{CH}_{2} \mathrm{OAc},{ }^{2} \mathrm{~J}_{\mathrm{H}, \mathrm{H}}=12.2 \mathrm{~Hz},{ }^{3} \mathrm{~J}_{\mathrm{H}, \mathrm{H}}=4.7$ $\mathrm{Hz}), 4.10\left(2 \mathrm{H}, \mathrm{dd}, \mathrm{CH}_{2} \mathrm{OAc},{ }^{2} \mathrm{~J}_{\mathrm{H}, \mathrm{H}}=12.2 \mathrm{~Hz},{ }^{3} J_{\mathrm{H}, \mathrm{H}}=2.3 \mathrm{~Hz}\right), 3.79-$ $3.82\left(4 \mathrm{H}, \mathrm{m}, 2 \mathrm{Ar}(\mathrm{C}=\mathrm{O}) \mathrm{OCH}_{2} \mathrm{CH}_{2} \mathrm{O}\right), 3.75\left(2 \mathrm{H}, \mathrm{ddd}, \mathrm{CHCH}_{2} \mathrm{OAc}\right.$, $\left.{ }^{3} J_{\mathrm{H}, \mathrm{H}}=10.0 \mathrm{~Hz},{ }^{3} J_{\mathrm{H}, \mathrm{H}}=4.7 \mathrm{~Hz},{ }^{3} J_{\mathrm{H}, \mathrm{H}}=2.3 \mathrm{~Hz}\right), 3.66-3.68(4 \mathrm{H}, \mathrm{m}$, $\left.2 \operatorname{Ar}(\mathrm{C}=\mathrm{O}) \mathrm{O}\left(\mathrm{CH}_{2}\right)_{2} \mathrm{OCH}_{2} \mathrm{CH}_{2}\right), 3.60-3.63(4 \mathrm{H}, \mathrm{m}, 2 \operatorname{Ar}(\mathrm{C}=\mathrm{O}) \mathrm{O}-$ $\left.\left(\mathrm{CH}_{2}\right)_{2} \mathrm{OCH}_{2} \mathrm{CH}_{2}\right), 3.62\left(4 \mathrm{H}, \quad \mathrm{s}, \quad 2 \mathrm{Ar}(\mathrm{C}=\mathrm{O}) \mathrm{O}\left(\mathrm{CH}_{2}\right)_{2} \mathrm{O}-\right.$ $\left.\left(\mathrm{CH}_{2}\right)_{2} \mathrm{OCH}_{2}\right), 2.04\left(6 \mathrm{H}, \mathrm{s}, 2 \mathrm{CH}_{2} \mathrm{O}(\mathrm{C}=\mathrm{O}) \mathrm{CH}_{3}\right), 2.00(6 \mathrm{H}, \mathrm{s}$, $\left.2(\mathrm{C}=\mathrm{O}) \mathrm{CH}_{3}\right), 1.96\left(6 \mathrm{H}, \mathrm{s}, 2(\mathrm{C}=\mathrm{O}) \mathrm{CH}_{3}\right), 1.89(6 \mathrm{H}, \mathrm{s}, 2(\mathrm{C}=$ O) $\left.\mathrm{CH}_{3}\right) .{ }^{31} \mathrm{P}$ NMR $\left(\mathrm{CDCl}_{3}\right): \delta_{\mathrm{P}} 38.73(2 \mathrm{P}) .{ }^{13} \mathrm{C} \mathrm{NMR}\left(\mathrm{CDCl}_{3}\right): \delta_{\mathrm{C}}$ $170.8\left(2 \mathrm{C}, 2(\mathrm{C}=\mathrm{O}) \mathrm{CH}_{3}\right), 170.4\left(2 \mathrm{C}, 2(\mathrm{C}=\mathrm{O}) \mathrm{CH}_{3}\right), 169.74(2 \mathrm{C}$, $\left.2(\mathrm{C}=\mathrm{O}) \mathrm{CH}_{3}\right), 169.67\left(2 \mathrm{C}, 2(\mathrm{C}=\mathrm{O}) \mathrm{CH}_{3}\right), 165.6(2 \mathrm{C}, 2 \mathrm{O}(\mathrm{C}=$ $\mathrm{O})(\mathrm{Ar}) \mathrm{CCHCHCP}), 135.4\left(2 \mathrm{C}, \mathrm{d}, 2 \mathrm{O}(\mathrm{C}=\mathrm{O})(\mathrm{Ar}) \mathrm{CCHCHCP},{ }^{1} \mathrm{~J}_{\mathrm{C}, \mathrm{P}}\right.$ $=54 \mathrm{~Hz}), 134.5\left(4 \mathrm{C}, \mathrm{d}, 4 \mathrm{P}(A r) \mathrm{CCHCHCH},{ }^{2} J_{\mathrm{C}, \mathrm{P}}=14 \mathrm{~Hz}\right), 134.4$ $\left(4 \mathrm{C}, \mathrm{d}, 4 \mathrm{P}(\mathrm{Ar}) \mathrm{CCHCHCH},{ }^{2} J_{\mathrm{C}, \mathrm{P}}=14 \mathrm{~Hz}\right), 134.1(4 \mathrm{C}, \mathrm{d}, 4 \mathrm{O}(\mathrm{C}=$ O) $\left.(A r) \mathrm{CCHCHCP},{ }^{2} \mathrm{~J}_{\mathrm{C}, \mathrm{P}}=14 \mathrm{~Hz}\right), 133.0(2 \mathrm{C}, \mathrm{d}, 2 \mathrm{O}(\mathrm{C}=\mathrm{O})(\mathrm{Ar})$ CCHCHCP, $\left.{ }^{4} J_{\mathrm{C}, \mathrm{P}}=3 \mathrm{~Hz}\right), 132.1\left(4 \mathrm{C}, \mathrm{d}, 4 \mathrm{P}(\mathrm{Ar}) \mathrm{CCHCHCH},{ }^{4} J_{\mathrm{C}, \mathrm{P}}=3\right.$ $\mathrm{Hz}), 130.1\left(4 \mathrm{C}, \mathrm{d}, 4 \mathrm{O}(\mathrm{C}=\mathrm{O})(\mathrm{Ar}) \mathrm{CCHCHCP},{ }^{3} J_{\mathrm{C}, \mathrm{P}}=12 \mathrm{~Hz}\right), 129.4$ $\left(8 \mathrm{C}, \mathrm{d}, 8 \mathrm{P}(A r) \mathrm{CCHCHCH},{ }^{3} J_{\mathrm{C}, \mathrm{P}}=12 \mathrm{~Hz}\right), 129.0(4 \mathrm{C}, \mathrm{d}, 4 \mathrm{P}(A r)$ $\left.\mathrm{CCHCHCH},{ }^{1} J_{\mathrm{C}, \mathrm{P}}=57 \mathrm{~Hz}\right), 83.2\left(2 \mathrm{C}, 2 \mathrm{CHCH}_{2} \mathrm{OAc}\right), 77.8(2 \mathrm{C}$, SCHCHCHOAc), 75.9 (2C, SCHCHOAc), 74.3 (2C, SCHCHOAc), $70.72,70.74\left(6 \mathrm{C}, 2(\mathrm{Ar})(\mathrm{C}=\mathrm{O}) \mathrm{O}\left(\mathrm{CH}_{2}\right)_{2} \mathrm{OCH}_{2}, 2(\mathrm{Ar})(\mathrm{C}=\mathrm{O}) \mathrm{O}-\right.$ $\left.\left(\mathrm{CH}_{2}\right)_{2} \mathrm{OCH}_{2} \mathrm{CH}_{2}, 2(\mathrm{Ar})(\mathrm{C}=\mathrm{O}) \mathrm{O}\left(\mathrm{CH}_{2}\right)_{2} \mathrm{O}\left(\mathrm{CH}_{2}\right)_{2} \mathrm{OCH}_{2}\right), \quad 69.2$ $\left(2 \mathrm{C}, 2(\mathrm{Ar})(\mathrm{C}=\mathrm{O}) \mathrm{OCH}_{2} \mathrm{CH}_{2} \mathrm{O}\right), 69.1\left(2 \mathrm{C}, 2 \mathrm{CHCHCH}_{2} \mathrm{OAc}\right), 64.7$ $\left(2 \mathrm{C}, 2(\mathrm{Ar})(\mathrm{C}=\mathrm{O}) \mathrm{OCH}_{2} \mathrm{CH}_{2} \mathrm{O}\right), 63.0\left(2 \mathrm{C}, 2 \mathrm{CHCH}_{2}(\mathrm{C}=\mathrm{O}) \mathrm{CH}_{3}\right)$, $21.2\left(2 \mathrm{C}, 2 \mathrm{CH}_{2} \mathrm{O}(\mathrm{C}=\mathrm{O}) \mathrm{CH}_{3}\right), 20.84\left(2 \mathrm{C}, 2(\mathrm{C}=\mathrm{O}) \mathrm{CH}_{3}\right), 20.80$ $\left(2 \mathrm{C}, 2(\mathrm{C}=\mathrm{O}) \mathrm{CH}_{3}\right), 20.75\left(2 \mathrm{C}, 2(\mathrm{C}=\mathrm{O}) \mathrm{CH}_{3}\right)$. ESI-MS $(+)$. Calcd for $\mathrm{C}_{76} \mathrm{H}_{86} \mathrm{Au}_{2} \mathrm{NaO}_{26} \mathrm{P}_{2} \mathrm{~S}_{2}^{+}: m / z$ 1957.3547 $\left([\mathrm{M}+\mathrm{Na}]^{+}\right)$. Found: $m / z$ 1957.3685. Calcd for $\mathrm{C}_{62} \mathrm{H}_{67} \mathrm{Au}_{2} \mathrm{O}_{17} \mathrm{P}_{2} \mathrm{~S}^{+}: \mathrm{m} / z$ 1571.2900 ([M $\left.\left.\mathrm{RS}^{-}\right]^{+}\right)$. Found: $m / z$ 1571.3116. UV-vis: $\lambda_{\max }=250 \mathrm{~nm}$.

Compound $6 d$. $\beta$-D-Thioglucosetetraacetate $(0.128 \mathrm{~g}, 0.351 \mathrm{mmol}$, 2 equiv), $\mathrm{K}_{2} \mathrm{CO}_{3}(0.053 \mathrm{~g}, 0.383 \mathrm{mmol}, 2.2$ equiv), and $6 \mathrm{c}(0.232 \mathrm{~g}$, $0.175 \mathrm{mmol}, 1$ equiv) in a mixture of $\mathrm{H}_{2} \mathrm{O} / \mathrm{EtOH} / \mathrm{CH}_{2} \mathrm{Cl}_{2}[30 \mathrm{~mL}$, 1:1:1 ( $/ \mathrm{v} / \mathrm{v})]$ were stirred for $72 \mathrm{~h}$ at $\mathrm{RT}$ in the dark. The reaction mixture was concentrated to dryness, and the crude was suspended in a mixture of acetone $/ \mathrm{CH}_{2} \mathrm{Cl}_{2}[30 \mathrm{~mL}, 1: 1(\mathrm{v} / \mathrm{v})]$. The inorganic salts were removed by filtration, and the filtrate was concentrated under reduced pressure and further dried under vacuum to afford the product as a white solid. Yield: $0.323 \mathrm{~g}, 0.163 \mathrm{mmol}, 94 \%$. Elem anal. Calcd for $\mathrm{C}_{78} \mathrm{H}_{90} \mathrm{Au}_{2} \mathrm{O}_{27} \mathrm{P}_{2} \mathrm{~S}_{2}$ : C, 47.33; $\mathrm{H}, 4.58$. Found: $\mathrm{C}, 47.43 ; \mathrm{H}, 4.55 .{ }^{1} \mathrm{H}$ NMR $\left(\mathrm{CDCl}_{3}\right): \delta_{\mathrm{H}} 8.10-8.13(4 \mathrm{H}, \mathrm{m}, 4 \mathrm{O}(\mathrm{C}=\mathrm{O})(\mathrm{Ar}) \mathrm{CCHCHCP}$, $\left.{ }^{3} J_{\mathrm{H}, \mathrm{H}}=8.4 \mathrm{~Hz},{ }^{4} J_{\mathrm{H}, \mathrm{H}}=2.0 \mathrm{~Hz}\right), 7.45-7.63(24 \mathrm{H}, \mathrm{m}, 4 \mathrm{O}(\mathrm{C}=$ $\mathrm{O})(A r) \mathrm{CCHCHCP}, 8 \mathrm{P}(A r) \mathrm{CCHCHCH}, 8 \mathrm{P}(A r) \mathrm{CCHCHCH}$, 4P$(A r) \mathrm{CCHCHCH}), 5.11-5.17(4 \mathrm{H}, \mathrm{m}, \mathrm{SCHCHOAc}$, $\mathrm{SCHCHCHOAc}), 5.08\left(2 \mathrm{H}, \mathrm{t}, \mathrm{CHCHCH}_{2} \mathrm{OAc},{ }^{3} J_{\mathrm{H}, \mathrm{H}}=9.6 \mathrm{~Hz}\right)$, $5.04\left(2 \mathrm{H}, \mathrm{t}, \mathrm{SCHCHOAc},{ }^{3} \mathrm{~J}_{\mathrm{H}, \mathrm{H}}=9.1 \mathrm{~Hz}\right), 4.46-4.49(4 \mathrm{H}, \mathrm{m}$, $\left.2 \mathrm{Ar}(\mathrm{C}=\mathrm{O}) \mathrm{OCH}_{2} \mathrm{CH}_{2} \mathrm{O}\right), 4.20\left(2 \mathrm{H}, \mathrm{dd}, \mathrm{CH}_{2} \mathrm{OAc},{ }^{2} J_{\mathrm{H}, \mathrm{H}}=12.2 \mathrm{~Hz}\right.$, $\left.{ }^{3} J_{\mathrm{H}, \mathrm{H}}=4.8 \mathrm{~Hz}\right), 4.10\left(2 \mathrm{H}, \mathrm{dd}, \mathrm{CH}_{2} \mathrm{OAc},{ }^{2} J_{\mathrm{H}, \mathrm{H}}=12.2 \mathrm{~Hz},{ }^{3} J_{\mathrm{H}, \mathrm{H}}=2.4\right.$ $\mathrm{Hz}), 3.80-3.82\left(4 \mathrm{H}, \mathrm{m}, 2 \mathrm{Ar}(\mathrm{C}=\mathrm{O}) \mathrm{OCH}_{2} \mathrm{CH}_{2} \mathrm{O}\right), 3.76(2 \mathrm{H}, \mathrm{ddd}$, $\left.\mathrm{CHCH}_{2} \mathrm{OAc},{ }^{3} J_{\mathrm{H}, \mathrm{H}}=9.8 \mathrm{~Hz},{ }^{3} J_{\mathrm{H}, \mathrm{H}}=4.8 \mathrm{~Hz},{ }^{3} J_{\mathrm{H}, \mathrm{H}}=2.8 \mathrm{~Hz}\right), 3.66-$ $3.68\left(4 \mathrm{H}, \mathrm{m}, 2 \mathrm{Ar}(\mathrm{C}=\mathrm{O}) \mathrm{O}\left(\mathrm{CH}_{2}\right)_{2} \mathrm{OCH}_{2} \mathrm{CH}_{2}\right), 3.62-3.64(4 \mathrm{H}, \mathrm{m}$, $\left.2 \mathrm{Ar}(\mathrm{C}=\mathrm{O}) \mathrm{O}\left(\mathrm{CH}_{2}\right)_{2} \mathrm{OCH}_{2} \mathrm{CH}_{2}\right), 3.60-3.63(8 \mathrm{H}, \mathrm{m}, 2 \mathrm{Ar}(\mathrm{C}=\mathrm{O}) \mathrm{O}-$ $\left.\left(\mathrm{CH}_{2}\right)_{2} \mathrm{O}\left(\mathrm{CH}_{2}\right)_{2} \mathrm{O}\left(\mathrm{CH}_{2}\right)_{2}\right), 2.04\left(6 \mathrm{H}, \mathrm{s}, 2 \mathrm{CH}_{2} \mathrm{O}(\mathrm{C}=\mathrm{O}) \mathrm{CH}_{3}\right), 2.00$ $\left(6 \mathrm{H}, \mathrm{s}, 2(\mathrm{C}=\mathrm{O}) \mathrm{CH}_{3}\right), 1.96\left(6 \mathrm{H}, \mathrm{s}, 2(\mathrm{C}=\mathrm{O}) \mathrm{CH}_{3}\right), 1.89(6 \mathrm{H}, \mathrm{s}$, $\left.2(\mathrm{C}=\mathrm{O}) \mathrm{CH}_{3}\right) .{ }^{31} \mathrm{P}$ NMR $\left(\mathrm{CDCl}_{3}\right): \delta_{\mathrm{P}} 38.78(2 \mathrm{P}) .{ }^{13} \mathrm{C} \quad \mathrm{NMR}$ $\left(\mathrm{CDCl}_{3}\right): \delta_{\mathrm{C}} 170.8\left(2 \mathrm{C}, 2(\mathrm{C}=\mathrm{O}) \mathrm{CH}_{3}\right), 170.4\left(2 \mathrm{C}, 2(\mathrm{C}=\mathrm{O}) \mathrm{CH}_{3}\right)$, $169.7\left(2 \mathrm{C}, 2(\mathrm{C}=\mathrm{O}) \mathrm{CH}_{3}\right), 169.6\left(2 \mathrm{C}, 2(\mathrm{C}=\mathrm{O}) \mathrm{CH}_{3}\right), 165.6(2 \mathrm{C}$, $2 \mathrm{O}(C=\mathrm{O})(A r) \mathrm{CCHCHCP}), 135.5(2 \mathrm{C}, \mathrm{d}, 2 \mathrm{O}(\mathrm{C}=\mathrm{O})(A r)-$ $\left.\mathrm{CCHCHCP},{ }^{1} J_{\mathrm{C}, \mathrm{P}}=53 \mathrm{~Hz}\right), 134.5\left(4 \mathrm{C}, \mathrm{d}, 4 \mathrm{P}(\mathrm{Ar}) \mathrm{CCHCHCH},{ }^{2} \mathrm{~J}_{\mathrm{C}, \mathrm{P}}\right.$ $=14 \mathrm{~Hz}), 134.4\left(4 \mathrm{C}, \mathrm{d}, 4 \mathrm{P}(\mathrm{Ar}) \mathrm{CCHCHCH},{ }^{2} \mathrm{~J}_{\mathrm{CP}}=14 \mathrm{~Hz}\right), 134.2$ $\left(4 \mathrm{C}, \mathrm{d}, 4 \mathrm{O}(\mathrm{C}=\mathrm{O})(\mathrm{Ar}) \mathrm{CCHCHCP},{ }^{2} J_{\mathrm{C}, \mathrm{P}}=14 \mathrm{~Hz}\right), 133.0(2 \mathrm{C}, \mathrm{d}$, $2 \mathrm{O}(\mathrm{C}=\mathrm{O})(A r)$ CСНCHCP, $\left.{ }^{4} J_{\mathrm{C}, \mathrm{P}}=2 \mathrm{~Hz}\right), 132.1(4 \mathrm{C}, \mathrm{m}, 4 \mathrm{P}(A r)-$ 
$\mathrm{CCHCHCH}), 130.1\left(4 \mathrm{C}, \mathrm{d}, 4 \mathrm{O}(\mathrm{C}=\mathrm{O})(\mathrm{Ar}) \mathrm{CCHCHCP},{ }^{3} J_{\mathrm{C}, \mathrm{P}}=11\right.$ $\mathrm{Hz}), 129.4\left(8 \mathrm{C}, \mathrm{d}, 8 \mathrm{P}(\mathrm{Ar}) \mathrm{CCHCHCH},{ }^{3} J_{\mathrm{C}, \mathrm{P}}=12 \mathrm{~Hz}\right), 129.0(4 \mathrm{C}, \mathrm{d}$, $\left.4 \mathrm{P}(\mathrm{Ar}) \mathrm{CCHCHCH},{ }^{1} J_{\mathrm{C}, \mathrm{P}}=56 \mathrm{~Hz}\right), 83.2\left(2 \mathrm{C}, 2 \mathrm{CHCH}_{2} \mathrm{OAc}\right), 77.8$ (2C, SCHCHCHOAc), 75.9 (2C, SCHCHOAc), 74.3 (2C, SCHCHOAc), 70.72, 70.70, $70.63\left(8 \mathrm{C}, 2(\mathrm{Ar})(\mathrm{C}=\mathrm{O}) \mathrm{O}\left(\mathrm{CH}_{2}\right)_{2} \mathrm{OCH}_{2}\right.$, $2(\mathrm{Ar})(\mathrm{C}=\mathrm{O}) \mathrm{O}\left(\mathrm{CH}_{2}\right)_{2} \mathrm{OCH}_{2} \mathrm{CH}_{2}, \quad 2(\mathrm{Ar})(\mathrm{C}=\mathrm{O}) \mathrm{O}\left(\mathrm{CH}_{2}\right)_{2} \mathrm{O}-$ $\left.\left(\mathrm{CH}_{2}\right)_{2} \mathrm{OCH}_{2}, 2(\mathrm{Ar})(\mathrm{C}=\mathrm{O}) \mathrm{O}\left(\mathrm{CH}_{2}\right)_{2} \mathrm{O}\left(\mathrm{CH}_{2}\right)_{2} \mathrm{OCH}_{2} \mathrm{CH}_{2}\right), 69.2$ $\left(2 \mathrm{C}, 2(\mathrm{Ar})(\mathrm{C}=\mathrm{O}) \mathrm{OCH}_{2} \mathrm{CH}_{2} \mathrm{O}\right), 69.1\left(2 \mathrm{C}, 2 \mathrm{CHCHCH}_{2} \mathrm{OAc}\right), 64.6$ $\left(2 \mathrm{C}, 2(\mathrm{Ar})(\mathrm{C}=\mathrm{O}) \mathrm{OCH}_{2} \mathrm{CH}_{2} \mathrm{O}\right), 62.9\left(2 \mathrm{C}, 2 \mathrm{CHCH}_{2}(\mathrm{C}=\mathrm{O}) \mathrm{CH}_{3}\right)$, $21.2\left(2 \mathrm{C}, 2 \mathrm{CH}_{2} \mathrm{O}(\mathrm{C}=\mathrm{O}) \mathrm{CH}_{3}\right), 20.80\left(2 \mathrm{C}, 2(\mathrm{C}=\mathrm{O}) \mathrm{CH}_{3}\right), 20.77$ (2C, $\left.2(\mathrm{C}=\mathrm{O}) \mathrm{CH}_{3}\right), 20.72\left(2 \mathrm{C}, 2(\mathrm{C}=\mathrm{O}) \mathrm{CH}_{3}\right)$. ESI-MS $(+)$. Calcd for $\mathrm{C}_{78} \mathrm{H}_{90} \mathrm{Au}_{2} \mathrm{NaO}_{27} \mathrm{P}_{2} \mathrm{~S}_{2}^{+}: m / z 2001.3809\left([\mathrm{M}+\mathrm{Na}]^{+}\right)$. Found: $m / z$ 2001.3790. Calcd for $\mathrm{C}_{64} \mathrm{H}_{71} \mathrm{Au}_{2} \mathrm{O}_{18} \mathrm{P}_{2} \mathrm{~S}^{+}: \mathrm{m} / z$ 1615.3162 ([M $\left.\mathrm{RS}^{-}\right]^{+}$). Found: $m / z$ 1615.3180. UV-vis: $\lambda_{\max }=250 \mathrm{~nm}$.

Compound 7d. $\beta$-D-Thioglucosetetraacetate $(0.130 \mathrm{~g}, 0.357 \mathrm{mmol}$, 2 equiv), $\mathrm{K}_{2} \mathrm{CO}_{3}(0.054 \mathrm{~g}, 0.391 \mathrm{mmol}, 2.2$ equiv), and $7 \mathrm{c}(0.252 \mathrm{~g}$, $0.178 \mathrm{mmol}, 1$ equiv) in a mixture of $\mathrm{H}_{2} \mathrm{O} / \mathrm{EtOH} / \mathrm{CH}_{2} \mathrm{Cl}_{2}[30 \mathrm{~mL}$, 1:1:1 ( $/ \mathrm{v} / \mathrm{v})]$ were stirred for $72 \mathrm{~h}$ at $\mathrm{RT}$ in the dark. The reaction mixture was concentrated to dryness, and the crude was suspended in a mixture of acetone $/ \mathrm{CH}_{2} \mathrm{Cl}_{2}[30 \mathrm{~mL}, 1: 1(\mathrm{v} / \mathrm{v})]$. The inorganic salts were removed by filtration, and the filtrate was concentrated under reduced pressure and further dried under vacuum to afford the product as a white solid. Yield: $0.317 \mathrm{~g}, 0.153 \mathrm{mmol}, 86 \%$. Elem anal. Calcd for $\mathrm{C}_{82} \mathrm{H}_{98} \mathrm{Au}_{2} \mathrm{O}_{29} \mathrm{P}_{2} \mathrm{~S}_{2}$ : C, 47.63; H, 4.78. Found: $\mathrm{C}, 47.85 ; \mathrm{H}, 4.60 .{ }^{1} \mathrm{H}$ NMR $\left(\mathrm{CDCl}_{3}\right): \delta_{\mathrm{H}} 8.10-8.13(4 \mathrm{H}, \mathrm{m}, 4 \mathrm{O}(\mathrm{C}=\mathrm{O})(\mathrm{Ar}) \mathrm{CCHCHCP}$, $\left.{ }^{3} J_{\mathrm{H}, \mathrm{H}}=8.4 \mathrm{~Hz},{ }^{4} J_{\mathrm{H}, \mathrm{H}}=2.0 \mathrm{~Hz}\right), 7.47-7.64(24 \mathrm{H}, \mathrm{m}, 4 \mathrm{O}(\mathrm{C}=$ $\mathrm{O})(\mathrm{Ar}) \mathrm{CCHCHCP}, 8 \mathrm{P}(\mathrm{Ar}) \mathrm{CCHCHCH}, 8 \mathrm{P}(\mathrm{Ar}) \mathrm{CCHCHCH}$, 4P$(A r) \mathrm{CCHCHCH}), 5.11-5.18(4 \mathrm{H}, \mathrm{m}, \mathrm{SCHCHOAc}$, SCHCHCHOAc), $5.09\left(2 \mathrm{H}, \mathrm{t}, \mathrm{CHCHCH}_{2} \mathrm{OAc},{ }^{3} \mathrm{~J}_{\mathrm{H}, \mathrm{H}}=9.6 \mathrm{~Hz}\right)$, $5.04\left(2 \mathrm{H}, \mathrm{t}, \mathrm{SCHCHOAc},{ }^{3} J_{\mathrm{H}, \mathrm{H}}=9.1 \mathrm{~Hz}\right), 4.47-4.49(4 \mathrm{H}, \mathrm{m}$, $\left.2 \operatorname{Ar}(\mathrm{C}=\mathrm{O}) \mathrm{OCH}_{2} \mathrm{CH}_{2} \mathrm{O}\right), 4.21\left(2 \mathrm{H}, \mathrm{dd}, \mathrm{CH}_{2} \mathrm{OAc},{ }^{2} J_{\mathrm{H}, \mathrm{H}}=12.2 \mathrm{~Hz}\right.$, $\left.{ }^{3} J_{\mathrm{H}, \mathrm{H}}=4.8 \mathrm{~Hz}\right), 4.10\left(2 \mathrm{H}, \mathrm{dd}, \mathrm{CH}_{2} \mathrm{OAc},{ }^{2} J_{\mathrm{H}, \mathrm{H}}=12.2 \mathrm{~Hz},{ }^{3} J_{\mathrm{H}, \mathrm{H}}=2.4\right.$ $\mathrm{Hz}), 3.80-3.83\left(4 \mathrm{H}, \mathrm{m}, 2 \mathrm{Ar}(\mathrm{C}=\mathrm{O}) \mathrm{OCH}_{2} \mathrm{CH}_{2} \mathrm{O}\right), 3.76(2 \mathrm{H}$, ddd, $\left.\mathrm{CHCH}_{2} \mathrm{OAc},{ }^{3} J_{\mathrm{H}, \mathrm{H}}=9.8 \mathrm{~Hz},{ }^{3} J_{\mathrm{H}, \mathrm{H}}=4.8 \mathrm{~Hz},{ }^{3} \mathrm{~J}_{\mathrm{H}, \mathrm{H}}=2.4 \mathrm{~Hz}\right), 3.66-$ $3.69\left(4 \mathrm{H}, \mathrm{m}, 2 \mathrm{Ar}(\mathrm{C}=\mathrm{O}) \mathrm{O}\left(\mathrm{CH}_{2}\right)_{2} \mathrm{OCH}_{2} \mathrm{CH}_{2}\right), 3.62-3.65(4 \mathrm{H}, \mathrm{m}$, $\left.2 \mathrm{Ar}(\mathrm{C}=\mathrm{O}) \mathrm{O}\left(\mathrm{CH}_{2}\right)_{2} \mathrm{OCH}_{2} \mathrm{CH}_{2}\right), 3.59-3.62(8 \mathrm{H}, \mathrm{m}, 2 \mathrm{Ar}(\mathrm{C}=\mathrm{O}) \mathrm{O}-$ $\left.\left(\mathrm{CH}_{2}\right)_{2} \mathrm{O}\left(\mathrm{CH}_{2}\right)_{2} \mathrm{O}\left(\mathrm{CH}_{2}\right)_{2}\right), 2.04\left(6 \mathrm{H}, \mathrm{s}, 2 \mathrm{CH}_{2} \mathrm{O}(\mathrm{C}=\mathrm{O}) \mathrm{CH}_{3}\right), 2.00$ $\left(6 \mathrm{H}, \mathrm{s}, 2(\mathrm{C}=\mathrm{O}) \mathrm{CH}_{3}\right), 1.97\left(6 \mathrm{H}, \mathrm{s}, 2(\mathrm{C}=\mathrm{O}) \mathrm{CH}_{3}\right), 1.90(6 \mathrm{H}, \mathrm{s}$, $\left.2(\mathrm{C}=\mathrm{O}) \mathrm{CH}_{3}\right) .{ }^{31} \mathrm{P}$ NMR $\left(\mathrm{CDCl}_{3}\right): \delta_{\mathrm{P}} 38.71(2 \mathrm{P}) .{ }^{13} \mathrm{C}$ NMR $\left(\mathrm{CDCl}_{3}\right): \delta_{\mathrm{C}} 170.8\left(2 \mathrm{C}, 2(\mathrm{C}=\mathrm{O}) \mathrm{CH}_{3}\right), 170.4\left(2 \mathrm{C}, 2(\mathrm{C}=\mathrm{O}) \mathrm{CH}_{3}\right)$, $169.71\left(2 \mathrm{C}, 2(\mathrm{C}=\mathrm{O})-\mathrm{CH}_{3}\right), 169.65\left(2 \mathrm{C}, 2(\mathrm{C}=\mathrm{O}) \mathrm{CH}_{3}\right), 165.6(2 \mathrm{C}$, $2 \mathrm{O}(\mathrm{C}=\mathrm{O})(A r) \mathrm{CCHCHCP}), 135.4 \quad(2 \mathrm{C}, \mathrm{d}, 2 \mathrm{O}(\mathrm{C}=\mathrm{O})(A r)-$ $\left.\mathrm{CCHCHCP},{ }^{1} J_{\mathrm{C}, \mathrm{P}}=53 \mathrm{~Hz}\right), 134.5\left(4 \mathrm{C}, \mathrm{d}, 4 \mathrm{P}(A r) \mathrm{CCHCHCH},{ }^{2} J_{\mathrm{C}, \mathrm{P}}\right.$ $=14 \mathrm{~Hz}), 134.4\left(4 \mathrm{C}, \mathrm{d}, 4 \mathrm{P}(\mathrm{Ar}) \mathrm{CCHCHCH},{ }^{2} J_{\mathrm{C}, \mathrm{P}}=14 \mathrm{~Hz}\right), 134.1$ $\left(4 \mathrm{C}, \mathrm{d}, 4 \mathrm{O}(\mathrm{C}=\mathrm{O})(\mathrm{Ar}) \mathrm{CCHCHCP},{ }^{2} J_{\mathrm{C}, \mathrm{P}}=14 \mathrm{~Hz}\right), 133.0(2 \mathrm{C}, \mathrm{d}$, $\left.2 \mathrm{O}(\mathrm{C}=\mathrm{O})(A r) C \mathrm{CHCHCP},{ }^{4} J_{\mathrm{C}, \mathrm{P}}=2 \mathrm{~Hz}\right), 132.1(4 \mathrm{C}, \mathrm{m}, 4 \mathrm{P}(A r)-$ $\mathrm{CCHCHCH}), 130.1\left(4 \mathrm{C}, \mathrm{d}, 4 \mathrm{O}(\mathrm{C}=\mathrm{O})(\mathrm{Ar}) \mathrm{CCHCHCP},{ }^{3} J_{\mathrm{C}, \mathrm{P}}=12\right.$ $\mathrm{Hz}), 129.4\left(8 \mathrm{C}, \mathrm{d}, 8 \mathrm{P}(\mathrm{Ar}) \mathrm{CCHCHCH},{ }^{3} J_{\mathrm{C}, \mathrm{P}}=12 \mathrm{~Hz}\right), 128.9(4 \mathrm{C}, \mathrm{d}$, $\left.4 \mathrm{P}(\mathrm{Ar}) \mathrm{CCHCHCH},{ }^{1} \mathrm{~J}_{\mathrm{C}, \mathrm{P}}=57 \mathrm{~Hz}\right), 83.2\left(2 \mathrm{C}, 2 \mathrm{CHCH}_{2} \mathrm{OAc}\right), 77.9$ (2C, SCHCHCHOAc), 75.8 (2C, SCHCHOAc), 74.2 (2C, SCHCHOAc), 70.70, 70.68, $70.61\left(8 \mathrm{C}, 2(\mathrm{Ar})(\mathrm{C}=\mathrm{O}) \mathrm{O}\left(\mathrm{CH}_{2}\right)_{2} \mathrm{OCH}_{2}\right.$, $2(A r)(\mathrm{C}=\mathrm{O}) \mathrm{O}\left(\mathrm{CH}_{2}\right)_{2} \mathrm{OCH}_{2} \mathrm{CH}_{2}, \quad 2(\mathrm{Ar})(\mathrm{C}=\mathrm{O}) \mathrm{O}\left(\mathrm{CH}_{2}\right)_{2} \mathrm{O}-$ $\left.\left(\mathrm{CH}_{2}\right)_{2} \mathrm{OCH}_{2}, 2(\mathrm{Ar})(\mathrm{C}=\mathrm{O}) \mathrm{O}\left(\mathrm{CH}_{2}\right)_{2} \mathrm{O}\left(\mathrm{CH}_{2}\right)_{2} \mathrm{OCH}_{2} \mathrm{CH}_{2}\right), 69.2$ (2C, $\left.2(\mathrm{Ar})(\mathrm{C}=\mathrm{O}) \mathrm{OCH}_{2} \mathrm{CH}_{2} \mathrm{O}\right), 69.0\left(2 \mathrm{C}, 2 \mathrm{CHCHCH}_{2} \mathrm{OAc}\right), 64.6$ (2C, $\left.2(\mathrm{Ar})(\mathrm{C}=\mathrm{O}) \mathrm{OCH}_{2} \mathrm{CH}_{2} \mathrm{O}\right), 62.9\left(2 \mathrm{C}, 2 \mathrm{CHCH}_{2}(\mathrm{C}=\mathrm{O}) \mathrm{CH}_{3}\right)$, $21.2\left(2 \mathrm{C}, 2 \mathrm{CH}_{2} \mathrm{O}(\mathrm{C}=\mathrm{O}) \mathrm{CH}_{3}\right), 20.82\left(2 \mathrm{C}, 2(\mathrm{C}=\mathrm{O}) \mathrm{CH}_{3}\right), 20.78$ $\left(2 \mathrm{C}, 2(\mathrm{C}=\mathrm{O}) \mathrm{CH}_{3}\right), 20.73\left(2 \mathrm{C}, 2(\mathrm{C}=\mathrm{O}) \mathrm{CH}_{3}\right)$. ESI-MS $(+)$. Calcd for $\mathrm{C}_{82} \mathrm{H}_{98} \mathrm{Au}_{2} \mathrm{NaO}_{29} \mathrm{P}_{2} \mathrm{~S}_{2}^{+}: m / z 2089.4334\left([\mathrm{M}+\mathrm{Na}]^{+}\right)$. Found: $m / z$ 2089.4307. Calcd for $\mathrm{C}_{68} \mathrm{H}_{79} \mathrm{Au}_{2} \mathrm{O}_{20} \mathrm{P}_{2} \mathrm{~S}^{+}: \mathrm{m} / z$ 1703.3686 ([M $\left.\mathrm{RS}^{-}\right]^{+}$). Found: $m / z$ 1703.3684. UV-vis: $\lambda_{\max }=250 \mathrm{~nm}$.

Compound $8 d$. According to the general procedure, $\beta$-Dthioglucosetetraacetate $(0.258 \mathrm{~g}, 0.708 \mathrm{mmol}, 1$ equiv $), \mathrm{K}_{2} \mathrm{CO}_{3}$ $(0.196 \mathrm{~g}, 1.418 \mathrm{mmol}, 2$ equiv), and $8 \mathrm{c}(0.350 \mathrm{~g}, 0.707 \mathrm{mmol}, 1$ equiv) in acetone $(25 \mathrm{~mL})$ were stirred for $24 \mathrm{~h}$ at $\mathrm{RT}$ in the dark. The product was isolated as a white solid. Yield: $0.548 \mathrm{~g}, 0.666 \mathrm{mmol}, 89 \%$. Elem anal. Calcd for $\mathrm{C}_{32} \mathrm{H}_{34} \mathrm{AuO}_{9} \mathrm{PS}$ : $\mathrm{C}, 46.72 ; \mathrm{H}, 4.17$. Found: $\mathrm{C}$, 46.97; $\mathrm{H}, 3.85 .{ }^{1} \mathrm{H}$ NMR $\left(\mathrm{CDCl}_{3}\right): \delta_{\mathrm{H}} 7.42-7.59(15 \mathrm{H}, \mathrm{m}$, 6P $(A r) \mathrm{CCHCHCH}, 6 \mathrm{P}(A r) \mathrm{CCHCHCH}, 3 \mathrm{P}(A r) \mathrm{CCHCHCH})$, 5.12-5.17 (2H, m, SCHCHOAc, SCHCHCHOAc), $5.08(1 \mathrm{H}, \mathrm{t}$, $\left.\mathrm{CHCHCH}_{2} \mathrm{OAc}^{3} J_{\mathrm{H}, \mathrm{H}}=9.5 \mathrm{~Hz}\right), 5.03\left(1 \mathrm{H}, \mathrm{t}, \mathrm{SCHCHOAc},{ }^{3} \mathrm{~J}_{\mathrm{H}, \mathrm{H}}=9.3\right.$
$\mathrm{Hz}), 4.19\left(1 \mathrm{H}, \mathrm{dd}, \mathrm{CH}_{2} \mathrm{OAc},{ }^{2} J_{\mathrm{H}, \mathrm{H}}=12.2 \mathrm{~Hz},{ }^{3} \mathrm{~J}_{\mathrm{H}, \mathrm{H}}=4.8 \mathrm{~Hz}\right), 4.09$ $\left(1 \mathrm{H}, \mathrm{dd}, \mathrm{CH}_{2} \mathrm{OAc},{ }^{2} J_{\mathrm{H}, \mathrm{H}}=12.2 \mathrm{~Hz},{ }^{3} J_{\mathrm{H}, \mathrm{H}}=2.4 \mathrm{~Hz}\right), 3.74(1 \mathrm{H}, \mathrm{ddd}$, $\left.\mathrm{CHCH}_{2} \mathrm{OAc}^{3} \mathrm{~J}_{\mathrm{H}, \mathrm{H}}=9.7 \mathrm{~Hz},{ }^{3} \mathrm{~J}_{\mathrm{H}, \mathrm{H}}=4.8 \mathrm{~Hz},{ }^{3} J_{\mathrm{H}, \mathrm{H}}=2.4 \mathrm{~Hz}\right), 2.02(3 \mathrm{H}$, s, $\left.\mathrm{CH}_{2} \mathrm{O}(\mathrm{C}=\mathrm{O}) \mathrm{CH}_{3}\right), 1.99\left(3 \mathrm{H}, \mathrm{s},(\mathrm{C}=\mathrm{O}) \mathrm{CH}_{3}\right), 1.95(3 \mathrm{H}, \mathrm{s},(\mathrm{C}=$ O) $\left.\mathrm{CH}_{3}\right), 1.87\left(3 \mathrm{H}, \mathrm{s},(\mathrm{C}=\mathrm{O}) \mathrm{CH}_{3}\right) \cdot{ }^{31} \mathrm{P}$ NMR $\left(\mathrm{CDCl}_{3}\right): \delta_{\mathrm{p}} 38.83$ (1P). ${ }^{13} \mathrm{C}$ NMR $\left(\mathrm{CDCl}_{3}\right): \delta_{\mathrm{C}} 170.9\left(1 \mathrm{C},(\mathrm{C}=\mathrm{O}) \mathrm{CH}_{3}\right), 170.4(1 \mathrm{C}$, $\left.(\mathrm{C}=\mathrm{O}) \mathrm{CH}_{3}\right), 169.7\left(1 \mathrm{C},(\mathrm{C}=\mathrm{O}) \mathrm{CH}_{3}\right), 169.7\left(1 \mathrm{C},(\mathrm{C}=\mathrm{O}) \mathrm{CH}_{3}\right)$, $134.4\left(6 \mathrm{C}, \mathrm{d}, 6 \mathrm{P}(\mathrm{Ar}) \mathrm{CCHCHCH},{ }^{2} J_{\mathrm{C}, \mathrm{P}}=14 \mathrm{~Hz}\right), 131.7(3 \mathrm{C}, \mathrm{d}$, $\left.3 \mathrm{P}(\mathrm{Ar}) \mathrm{CCHCHCH},{ }^{4} J_{\mathrm{C}, \mathrm{P}}=2 \mathrm{~Hz}\right), 129.8(3 \mathrm{C}, \mathrm{d}, 3 \mathrm{P}(\mathrm{Ar}) \mathrm{CCHCHCH}$, $\left.{ }^{1} J_{\mathrm{C}, \mathrm{P}}=57 \mathrm{~Hz}\right), 129.3\left(6 \mathrm{C}, \mathrm{d}, 6 \mathrm{P}(\mathrm{Ar}) \mathrm{CCHCHCH},{ }^{3} J_{\mathrm{C}, \mathrm{P}}=11 \mathrm{~Hz}\right), 83.2$ (1C, $\mathrm{CHCH}_{2} \mathrm{OAc}$ ), 77.8 (1C, SCHCHCHOAc), 75.8 (1C, SCHCHOAc), 74.3 (1C, SCHCHOAc), 69.1 (1C, $\mathrm{CHCHCH}_{2} \mathrm{OAc}$ ), $63.0\left(1 \mathrm{C}, \mathrm{CHCH}_{2}(\mathrm{C}=\mathrm{O}) \mathrm{CH}_{3}\right), 21.2\left(1 \mathrm{C}, \mathrm{CH}_{2} \mathrm{O}(\mathrm{C}=\mathrm{O}) \mathrm{CH}_{3}\right), 20.80$ $\left(2 \mathrm{C}, 2(\mathrm{C}=\mathrm{O}) \mathrm{CH}_{3}\right), 20.75\left(1 \mathrm{C},(\mathrm{C}=\mathrm{O}) \mathrm{CH}_{3}\right)$. ESI-MS $(+)$. Calcd for $\mathrm{C}_{32} \mathrm{H}_{34} \mathrm{AuO}_{9} \mathrm{PS}: m / z$ 822.1327 $\left([\mathrm{M}+\mathrm{H}]^{+}\right)$. Found: $m / z 823.1407$. Calcd for $\mathrm{C}_{32} \mathrm{H}_{34} \mathrm{AuNaO}_{9} \mathrm{PS}^{+}: m / z 845.1224\left([\mathrm{M}+\mathrm{Na}]^{+}\right)$. Found: $m /$ $z$ 845.1239.

Compound 9d. According to the general procedure, $\beta$-Dthioglucosetetraacetate $\left(0.304 \mathrm{~g}, 0.834 \mathrm{mmol}, 1\right.$ equiv), $\mathrm{K}_{2} \mathrm{CO}_{3}$ (0.231 g, $1.671 \mathrm{mmol}, 2$ equiv), and $9 \mathrm{c}(0.450 \mathrm{~g}, 0.835 \mathrm{mmol}, 1$ equiv) in acetone $(35 \mathrm{~mL})$ were stirred for $48 \mathrm{~h}$ at RT in the dark. The product was isolated as a white solid. Yield: $0.687 \mathrm{~g}, 0.793 \mathrm{mmol}, 95 \%$. Elem anal. Calcd for $\mathrm{C}_{33} \mathrm{H}_{34} \mathrm{AuO}_{11} \mathrm{PS} \cdot \mathrm{CDCl}_{3}$ : C, 40.56; H, 3.70. Found: $\mathrm{C}, 39.96 ; \mathrm{H}, 3.57 . \mathrm{CDCl}_{3}$ originates from the NMR solvent. ${ }^{1} \mathrm{H}$ $\operatorname{NMR}\left(\mathrm{CDCl}_{3}\right): \delta_{\mathrm{H}} 7.81-7.90(2 \mathrm{H}, \mathrm{m}, 2 \mathrm{O}(\mathrm{C}=\mathrm{O})(\mathrm{Ar}) \mathrm{CCHCHCP})$, $7.30-7.48(12 \mathrm{H}, \quad \mathrm{m}, \quad 2 \mathrm{O}(\mathrm{C}=\mathrm{O})(A r) \mathrm{CCHCHCP}, 4 \mathrm{P}(A r)$ $\mathrm{CCHCHCH}, 4 \mathrm{P}(\mathrm{Ar}) \mathrm{CCHCHCH}, 2 \mathrm{P}(\mathrm{Ar}) \mathrm{CCHCHCH}), \quad 5.06-5.13$ $(2 \mathrm{H}, \mathrm{m}, \mathrm{SCHCHOAc}, \mathrm{SCHCHCHOAc}), 4.99(1 \mathrm{H}, \mathrm{t}$, $\left.\mathrm{CHCHCH}_{2} \mathrm{OAc},{ }^{3} J_{\mathrm{H}, \mathrm{H}}=9.2 \mathrm{~Hz}\right), 4.97\left(1 \mathrm{H}, \mathrm{t}, \mathrm{SCHCHOAc},{ }^{3} \mathrm{~J}_{\mathrm{H}, \mathrm{H}}=\right.$ $9.3 \mathrm{~Hz}), 4.07\left(1 \mathrm{H}, \mathrm{dd}, \mathrm{CH}_{2} \mathrm{OAc},{ }^{2} J_{\mathrm{H}, \mathrm{H}}=12.1 \mathrm{~Hz},{ }^{3} J_{\mathrm{H}, \mathrm{H}}=5.1 \mathrm{~Hz}\right), 4.02$ $\left(1 \mathrm{H}, \mathrm{d}, \mathrm{CH}_{2} \mathrm{OAc},{ }^{2} J_{\mathrm{H}, \mathrm{H}}=12.1 \mathrm{~Hz}\right), 3.68\left(1 \mathrm{H}, \mathrm{m}, \mathrm{CHCH}_{2} \mathrm{OAc}\right), 2.16$ $\left(6 \mathrm{H}, \mathrm{s}, 2 \mathrm{CH}_{2} \mathrm{O}(\mathrm{C}=\mathrm{O}) \mathrm{CH}_{3}\right), 2.00\left(3 \mathrm{H}, \mathrm{s},(\mathrm{C}=\mathrm{O}) \mathrm{CH}_{3}\right), 1.91(3 \mathrm{H}, \mathrm{s}$, $\left.(\mathrm{C}=\mathrm{O}) \mathrm{CH}_{3}\right), 1.90\left(3 \mathrm{H}, \mathrm{s},(\mathrm{C}=\mathrm{O}) \mathrm{CH}_{3}\right) \cdot{ }^{31} \mathrm{P} \mathrm{NMR}\left(\mathrm{CDCl}_{3}\right): \delta_{\mathrm{P}}$ $38.82(1 \mathrm{P}) .{ }^{13} \mathrm{C}$ NMR $\left(\mathrm{CDCl}_{3}\right): \delta_{\mathrm{C}} 171.8\left(1 \mathrm{C},(\mathrm{C}=\mathrm{O}) \mathrm{CH}_{3}\right), 171.4$ $\left(1 \mathrm{C}, \quad(\mathrm{C}=\mathrm{O}) \mathrm{CH}_{3}\right), 170.2\left(1 \mathrm{C}, \quad(\mathrm{C}=\mathrm{O}) \mathrm{CH}_{3}\right), 170.1 \quad(1 \mathrm{C}, \quad(\mathrm{C}=$ $\left.\mathrm{O}) \mathrm{CH}_{3}\right), 169.8(1 \mathrm{C}, \mathrm{O}(\mathrm{C}=\mathrm{O})(\mathrm{Ar}) \mathrm{CCHCHCP}), 141.7(1 \mathrm{C}, \mathrm{m}$, $\mathrm{O}(\mathrm{C}=\mathrm{O})(\mathrm{Ar}) \mathrm{CCHCHCP}), 134.3\left(2 \mathrm{C}, \mathrm{d}, 2 \mathrm{P}(\mathrm{Ar}) \mathrm{CCHCHCH},{ }^{2} J_{\mathrm{C}, \mathrm{P}}=\right.$ $15 \mathrm{~Hz}), 134.2\left(2 \mathrm{C}, \mathrm{d}, 2 \mathrm{P}(\mathrm{Ar}) \mathrm{CCHCHCH},{ }^{2} J_{\mathrm{C}, \mathrm{P}}=15 \mathrm{~Hz}\right), 133.7(2 \mathrm{C}$, $\left.\mathrm{d}, 2 \mathrm{O}(\mathrm{C}=\mathrm{O})(\mathrm{Ar}) \mathrm{CCHCHCP},{ }^{2} \mathrm{~J}_{\mathrm{C}, \mathrm{P}}=14 \mathrm{~Hz}\right), 131.9(3 \mathrm{C}, \mathrm{m}, \mathrm{O}(\mathrm{C}=$ O) $(A r) C C H C H C P, 2 P(A r) \mathrm{CCHCHCH}), 129.9(2 \mathrm{C}, \mathrm{m}, 2 \mathrm{P}(A r)$ $\mathrm{CCHCHCH}), 129.7\left(2 \mathrm{C}, \mathrm{d}, 2 \mathrm{O}(\mathrm{C}=\mathrm{O})(\mathrm{Ar}) \mathrm{CCHCHCP},{ }^{3} J_{\mathrm{C}, \mathrm{P}}=11\right.$ $\mathrm{Hz}), 129.4\left(2 \mathrm{C}, \mathrm{d}, 2 \mathrm{P}(\mathrm{Ar}) \mathrm{CCHCHCH},{ }^{3} \mathrm{~J}_{\mathrm{C}, \mathrm{P}}=11 \mathrm{~Hz}\right), 129.3(2 \mathrm{C}, \mathrm{d}$, $\left.2 \mathrm{P}(\mathrm{Ar}) \mathrm{CCHCHCH},{ }^{3} \mathrm{~J}_{\mathrm{C}, \mathrm{P}}=12 \mathrm{~Hz}\right), 83.2\left(1 \mathrm{C}, \mathrm{CHCH}_{2} \mathrm{OAc}\right), 77.5$ (1C, SCHCHCHOAc), 75.6 (1C, SCHCHOAc), 74.1 (1C, $\mathrm{SCHCHOAc}), 69.3$ (1C, $\left.\mathrm{CHCHCH}_{2} \mathrm{OAc}\right), 63.3\left(1 \mathrm{C}, \mathrm{CHCH}_{2}(\mathrm{C}=\right.$ $\left.\mathrm{O}) \mathrm{CH}_{3}\right), 21.2\left(1 \mathrm{C}, \mathrm{CH}_{2} \mathrm{O}(\mathrm{C}=\mathrm{O}) \mathrm{CH}_{3}\right), 20.8\left(2 \mathrm{C}, 2(\mathrm{C}=\mathrm{O}) \mathrm{CH}_{3}\right)$, $20.7\left(1 \mathrm{C},(\mathrm{C}=\mathrm{O}) \mathrm{CH}_{3}\right)$. ESI-MS $(+)$. Calcd for $\mathrm{C}_{33} \mathrm{H}_{34} \mathrm{AuO}_{11} \mathrm{PS}: \mathrm{m} / z$ 866.1225 $\left([\mathrm{M}+\mathrm{H}]^{+}\right)$. Found: $m / z$ 867.1310. Calcd for $\mathrm{C}_{33} \mathrm{H}_{34} \mathrm{AuNaO}_{11} \mathrm{PS}^{+}: m / z$ 889.1123 $\left([\mathrm{M}+\mathrm{Na}]^{+}\right)$. Found: $m / z$ 889.1143. Calcd for $\mathrm{C}_{33} \mathrm{H}_{34} \mathrm{AuKO}_{11} \mathrm{PS}^{+}: m / z 905.0862\left([\mathrm{M}+\mathrm{K}]^{+}\right)$. Found: $m / z$ 905.0881.

Stability Studies. The stability of complexes $\mathbf{1 b}-7 \mathbf{b}$ and $\mathbf{1 d}-\mathbf{7 d}$ in DMSO- $d^{6}$ was assessed via ${ }^{1} \mathrm{H}(400 \mathrm{MHz})$ and ${ }^{31} \mathrm{P}(162 \mathrm{MHz}) \mathrm{NMR}$ at $298 \mathrm{~K}$ for $20 \mathrm{~min}$. The stability of complexes $2 \mathrm{~d}, \mathbf{4 d}$, and $\mathbf{6 d}$ in pseudocell culture conditions was assessed in aqueous $100 \mathrm{mM} \mathrm{NaCl}$ and 5\% DMSO for $72 \mathrm{~h}$ at $298 \mathrm{~K}$ and monitored via ESI-MS(+).

Cell Culture and in Vitro Antiproliferative Activity. The human ovarian carcinoma (A2780 and A2780cisR) cell lines were obtained from the European Collection of Cell Cultures. The human embryonic kidney (HEK-293) cell line was obtained from ATCC (Sigma, Buchs, Switzerland). Penicillin streptomycin, RPMI 1640 GlutaMAX (where RPMI = Roswell Park Memorial Institute), and DMEM GlutaMAX media (where DMEM = Dulbecco's modified Eagle medium) were obtained from Life Technologies, and fetal bovine serum (FBS) was obtained from Sigma. The cells were cultured in RPMI 1640 GlutaMAX (A2780 and A2780cisR) and DMEM GlutaMAX (HEK-293) media containing 10\% heat-inactivated FBS and $1 \%$ penicillin streptomycin at $37{ }^{\circ} \mathrm{C}$ and $\mathrm{CO}_{2}$ (5\%).The A2780cisR cell line was routinely treated with cisplatin $(2 \mu \mathrm{M})$ in the media. The cytotoxicity was determined using the 3-(4,5-dimethyl- 
2-thiazolyl)-2,5-diphenyl-2H-tetrazolium bromide (MTT) assay. ${ }^{83}$ Cells were seeded in flat-bottomed 96-well plates as a suspension in a prepared medium (100 $\mu \mathrm{L}$ aliquots and approximately 4300 cells/ well) and preincubated for $24 \mathrm{~h}$. Stock solutions of compounds were prepared in DMSO and were rapidly diluted in a medium. The solutions were sequentially diluted to give a final DMSO concentration of $0.5 \%$ and a final compound concentration range $(0-500 \mu \mathrm{M})$. Cisplatin was tested as a positive control $(0-100 \mu \mathrm{M})$. The compounds were added to the preincubated 96-well plates in 100 $\mu \mathrm{L}$ aliquots, and the plates were incubated for $72 \mathrm{~h}$. MTT $(20 \mu \mathrm{L}, 5$ $\mathrm{mg} / \mathrm{mL}$ in Dulbecco's phosphate buffered saline) was added to the cells, and the plates were incubated for a further $4 \mathrm{~h}$. The culture medium was aspirated, and the purple formazan crystals, formed by the mitochondrial dehydrogenase activity of vital cells, were dissolved in DMSO (100 $\mu \mathrm{L} /$ well). The absorbance of the resulting solutions, directly proportional to the number of surviving cells, was quantified at $590 \mathrm{~nm}$ using a microplate reader. The percentage of surviving cells was calculated from the absorbance of wells corresponding to the untreated control cells. The reported $\mathrm{IC}_{50}$ values (Table 3 ) are based on the means from three independent experiments, each comprising four tests per concentration level.

\section{ASSOCIATED CONTENT}

\section{S Supporting Information}

The Supporting Information is available free of charge on the ACS Publications website at DOI: 10.1021/acs.inorgchem.7b01082.

Crystal data and refinement for $9 \mathbf{b}$ and $9 c$, partition coefficient data, NMR spectroscopy stability data for $\mathbf{1 b}-\mathbf{7 b}$ and $\mathbf{1 d - 7 d , ~ E S I - M S ( + ) ~ s t a b i l i t y ~ d a t a ~ o f ~} \mathbf{2 d}, \mathbf{4 d}$, and $\mathbf{6 d}$, and NMR spectra of all compounds (PDF)

\section{Accession Codes}

CCDC 1542726 and 1542742 contain the supplementary crystallographic data for this paper. These data can be obtained free of charge via www.ccdc.cam.ac.uk/data_request/cif, or by emailing data_request@ccdc.cam.ac.uk, or by contacting The Cambridge Crystallographic Data Centre, 12 Union Road, Cambridge CB2 1EZ, UK; fax: +44 1223336033.

\section{AUTHOR INFORMATION}

\section{Corresponding Author}

*E-mail: paul.dyson@epfl.ch.

ORCID

Paul J. Dyson: 0000-0003-3117-3249

Notes

The authors declare no competing financial interest.

\section{ACKNOWLEDGMENTS}

We thank the Swiss National Science Foundation for financial support and Dr. Euro Solari (EPFL) for elemental analysis and assistance with crystal structure refinement.

\section{REFERENCES}

(1) Johnstone, T. C.; Suntharalingam, K.; Lippard, S. J. The Next Generation of Platinum Drugs: Targeted Pt(II) Agents, Nanoparticle Delivery, and Pt(IV) Prodrugs. Chem. Rev. 2016, 116, 3436.

(2) Jakupec, M. A.; Galanski, M.; Arion, V. B.; Hartinger, C. G.; Keppler, B. K. Antitumour metal compounds: more than theme and variations. Dalton Trans. 2008, 2, 183.

(3) Leijen, S.; Burgers, S. A.; Baas, P.; Pluim, D.; Tibben, M.; van Werkhoven, E.; Alessio, E.; Sava, G.; Beijnen, J. H.; Schellens, J. H. M. Phase I/II study with ruthenium compound NAMI-A and gemcitabine in patients with non-small cell lung cancer after first line therapy. Invest. New Drugs 2015, 33, 201.
(4) Hartinger, C. G.; Zorbas-Seifried, S.; Jakupec, M. A.; Kynast, B.; Zorbas, H.; Keppler, B. K. From bench to bedside-preclinical and early clinical development of the anticancer agent indazolium trans[tetrachlorobis(1H-indazole)ruthenate(III)] (KP1019 or FFC14A). J. Inorg. Biochem. 2006, 100, 891.

(5) Hartinger, C. G.; Jakupec, M. A.; Zorbas-Seifried, S.; Groessl, M.; Egger, A.; Berger, W.; Zorbas, H.; Dyson, P. J.; Keppler, B. K. KP1019, A New Redox-Active Anticancer Agent - Preclinical Development and Results of a Clinical Phase I Study in Tumor Patients. Chem. Biodiversity 2008, 5, 2140.

(6) Lentz, F.; Drescher, A.; Lindauer, A.; Henke, M.; Hilger, R. A.; Hartinger, C. G.; Scheulen, M. E.; Dittrich, C.; Keppler, B. K.; Jaehde, $\mathrm{U}$. Pharmacokinetics of a novel anticancer ruthenium complex (KP1019, FFC14A) in a phase I dose-escalation study. Anti-Cancer Drugs 2009, 20, 97.

(7) Trondl, R; Heffeter, P.; Kowol, C. R.; Jakupec, M. A.; Berger, W.; Keppler, B. K. NKP-1339, the first ruthenium-based anticancer drug on the edge to clinical application. Chem. Sci. 2014, 5, 2925.

(8) Singh, A. K.; Pandey, D. S.; Xu, Q.; Braunstein, P. Recent advances in supramolecular and biological aspects of arene ruthenium (II) complexes. Coord. Chem. Rev. 2014, 270-271, 31.

(9) Ang, W. H.; Casini, A.; Sava, G.; Dyson, P. J. Organometallic ruthenium-based antitumor compounds with novel modes of action. J. Organomet. Chem. 2011, 696, 989.

(10) Nazarov, A. A.; Hartinger, C. G.; Dyson, P. J. Opening the lid on piano-stool complexes: An account of ruthenium(II)-arene complexes with medicinal applications. J. Organomet. Chem. 2014, 751, 251.

(11) Murray, B. S.; Babak, M. V.; Hartinger, C. G.; Dyson, P. J. The development of RAPTA compounds for the treatment of tumors. Coord. Chem. Rev. 2016, 306, 86.

(12) Habtemariam, A.; Melchart, M.; Fernández, R.; Parsons, S.; Oswald, I. D. H.; Parkin, A.; Fabbiani, F. P. A.; Davidson, J. E.; Dawson, A.; Aird, R. E.; Jodrell, D. I.; Sadler, P. J. Structure-Activity Relationships for Cytotoxic Ruthenium(II) Arene Complexes Containing N,N-, N,O-, and O,O-Chelating Ligands. J. Med. Chem. 2006, 49, 6858 .

(13) Allardyce, C. S.; Dyson, P. J.; Ellis, D. J.; Salter, P. A.; Scopelliti, R. Synthesis and characterisation of some water soluble ruthenium(II)-arene complexes and an investigation of their antibiotic and antiviral properties. J. Organomet. Chem. 2003, 668, 35.

(14) Morris, R. E.; Aird, R. E.; del Socorro Murdoch, P.; Chen, H.; Cummings, J.; Hughes, N. D.; Parsons, S.; Parkin, A.; Boyd, G.; Jodrell, D. I.; Sadler, P. J. Inhibition of cancer cell growth by ruthenium(II) arene complexes. J. Med. Chem. 2001, 44, 3616.

(15) Scolaro, C.; Bergamo, A.; Brescacin, L.; Delfino, R.; Cocchietto, M.; Laurenczy, G.; Geldbach, T. J.; Sava, G.; Dyson, P. J. In vitro and in vivo evaluation of ruthenium(II)-arene PTA complexes. J. Med. Chem. 2005, 48, 4161.

(16) Weiss, A.; Berndsen, R. H.; Dubois, M.; Muller, C.; Schibli, R.; Griffioen, A. W.; Dyson, P. J.; Nowak-Sliwinska, P. In vivo anti-tumor activity of the organometallic ruthenium(II)-arene complex $\left[\mathrm{Ru}\left(\eta_{6}-\mathrm{p}\right.\right.$ cymene) $\mathrm{Cl}_{2}$ (pta)] (RAPTA-C) in human ovarian and colorectal carcinomas. Chem. Sci. 2014, 5, 4742.

(17) Nowak-Sliwinska, P.; van Beijnum, J. R.; Casini, A.; Nazarov, A. A.; Wagnieres, G.; van den Bergh, H.; Dyson, P. J.; Griffioen, A. W. Organometallic ruthenium(II) arene compounds with antiangiogenic activity. J. Med. Chem. 2011, 54, 3895.

(18) Weiss, A.; Ding, X.; van Beijnum, J. R.; Wong, I.; Wong, T. J.; Berndsen, R. H.; Dormond, O.; Dallinga, M.; Shen, L.; Schlingemann, R. O.; Pili, R.; Ho, C. M.; Dyson, P. J.; van den Bergh, H.; Griffioen, A. W.; Nowak-Sliwinska, P. Rapid optimization of drug combinations for the optimal angiostatic treatment of cancer. Angiogenesis 2015, 18, 233.

(19) Bergamo, A.; Masi, A.; Peacock, A. F. A.; Habtemariam, A.; Sadler, P. J.; Sava, G. In vivo tumour and metastasis reduction and in vitro effects on invasion assays of the ruthenium RM175 and osmium AFAP51 organometallics in the mammary cancer model. J. Inorg. Biochem. 2010, 104, 79.

(20) Adhireksan, Z.; Davey, G. E.; Campomanes, P.; Groessl, M.; Clavel, C. M.; Yu, H.; Nazarov, A. A.; Yeo, C. H. F.; Ang, W. H.; 
Dröge, P.; Rothlisberger, U.; Dyson, P. J.; Davey, C. A. Ligand substitutions between ruthenium-cymene compounds can control protein versus DNA targeting and anticancer activity. Nat. Commun. 2014, 5, 3462.

(21) Palermo, G.; Magistrato, A.; Riedel, T.; von Erlach, T.; Davey, C. A.; Dyson, P. J.; Rothlisberger, U. Fighting Cancer with Transition Metal Complexes: From Naked DNA to Protein and Chromatin Targeting Strategies. ChemMedChem 2016, 11, 1199.

(22) Tiekink, E. R. T. Gold derivatives for the treatment of cancer. Crit. Rev. Oncol. Hematol. 2002, 42, 225.

(23) Tiekink, E. R.T. Anti-cancer potential of gold complexes. Inflammopharmacology 2008, 16, 138.

(24) Mirabelli, C. K.; Johnson, R. K.; Hill, D. T.; Faucette, L. F.; Girard, G. R.; Kuo, G. Y.; Sung, C. M.; Crooke, S. T. Correlation of the in vitro cytotoxic and in vivo antitumor activities of gold (I) coordination complexes. J. Med. Chem. 1986, 29, 218.

(25) Messori, L.; Marcon, G. Gold Complexes for the Treatment of Rheumatoid Arthritus. Met. Ions Biol. Syst. 2004, 41, 279.

(26) Kean, W. F.; Hart, L.; Buchanan, W. W. Auranofin. Rheumatology 1997, 36, 560.

(27) Mirabelli, C. K.; Johnson, R. K.; Sung, C. M.; Faucette, L.; Muirhead, K.; Crooke, S. T. Evaluation of the In Vivo Antitumor Activity and In Vitro Cytotoxic Properties of Auranofin, a Coordinated Gold Compound, in Murine Tumor Models. Cancer Res. 1985, 45, 32.

(28) Madeira, J. M.; Gibson, D. L.; Kean, W. F.; Klegeris, A. The biological activity of auranofin: implications for novel treatment of diseases. Inflammopharmacology 2012, 20, 297.

(29) Liu, C.; Liu, Z.; Li, M.; Li, X.; Wong, Y. S.; Ngai, S. M.; Zheng, W.; Zhang, Y.; Chen, T. Enhancement of auranofin-induced apoptosis in MCF-7 human breast cells by selenocystine, a synergistic inhibitor of thioredoxin reductase. PLoS One 2013, 8, e53945.

(30) Park, S. H.; Lee, J. H.; Berek, J. S.; Hu, M. C. T Auranofin displays anticancer activity against ovarian cancer cells through FOXO3 activation independent of p53. Int. J. Oncol. 2014, 45, 1691.

(31) Fan, C.; Zheng, W.; Fu, X.; Li, X.; Wong, Y. S.; Chen, T. Enhancement of auranofin-induced lung cancer cell apoptosis by selenocystine, a natural inhibitor of TrxR1 in vitro and in vivo. Cell Death Dis. 2014, 5, e1191.

(32) Li, H.; Hu, J.; Wu, S.; Wang, L.; Cao, X.; Zhang, X.; Dai, B.; Cao, M.; Shao, R.; Zhang, R.; et al. Auranofin-mediated inhibition of $\mathrm{PI} 3 \mathrm{~K} / \mathrm{AKT} / \mathrm{mTOR}$ axis and anticancer activity in non-small cell lung cancer cells. Oncotarget 2016, 7, 3548 .

(33) Casini, A.; Gabbiani, C.; Sorrentino, F.; Rigobello, M. P.; Bindoli, A.; Geldbach, T. J.; Marrone, A.; Re, N.; Hartinger, C. G.; Dyson, P. J.; Messori, L. Emerging Protein Targets for Anticancer Metallodrugs: Inhibition of Thioredoxin Reductase and Cathepsin B by Antitumor Ruthenium(II)-Arene Compounds. J. Med. Chem. 2008, 51, 6773.

(34) Ang, W. H.; Parker, L. J.; De Luca, A.; Juillerat-Jeanneret, L.; Morton, C. J.; Lo Bello, M.; Parker, M. W.; Dyson, P. J. Rational Design of an Organometallic Glutathione Transferase Inhibitor. Angew. Chem., Int. Ed. 2009, 48, 3854.

(35) Barnard, P. J.; Berners-Price, S. J. Targeting the mitochondrial cell death pathway with gold compounds. Coord. Chem. Rev. 2007, 251, 1889.

(36) Zou, T.; Lum, C. T.; Lok, C. N.; Zhang, J. J.; Che, C. M. Chemical biology of anticancer gold(iii) and gold(i) complexes. Chem. Soc. Rev. 2015, 44, 8786.

(37) Roder, C.; Thomson, M. J. Auranofin: Repurposing an Old Drug for a Golden New Age. Drugs $R \& D$ 2015, 15, 13.

(38) Hartinger, C. G.; Phillips, A. D.; Nazarov, A. A. Polynuclear ruthenium, osmium and gold complexes. The quest for innovative anticancer chemotherapeutics. Curr. Top. Med. Chem. 2011, 11, 2688.

(39) Manzotti, C.; Pratesi, G.; Menta, E.; Di Domenico, R.; Cavalletti, E.; Fiebig, H. H.; Kelland, L. R.; Farrell, N.; Polizzi, D.; Supino, R.; Pezzoni, G.; Zunino, F. BBR 3464: A Novel Triplatinum Complex, Exhibiting a Preclinical Profile of Antitumor Efficacy Different from Cisplatin. Clin. Cancer Res. 2000, 6, 2626.
(40) Jodrell, D. I.; Evans, T. R. J.; Steward, W.; Cameron, D.; Prendiville, J.; Aschele, C.; Noberasco, C.; Lind, M.; Carmichael, J.; Dobbs, N.; Camboni, G.; Gatti, B.; De Braud, F. Phase II studies of BBR3464, a novel tri-nuclear platinum complex, in patients with gastric or gastro-oesophageal adenocarcinoma. Eur. J. Cancer 2004, 40, 1872.

(41) Hensing, T. A.; Hanna, N. H.; Gillenwater, H. H.; Camboni, M. G.; Allievi, C.; Socinski, M. A. Phase II study of BBR 3464 as treatment in patients with sensitive or refractory small cell lung cancer. AntiCancer Drugs 2006, 17, 697.

(42) Stringer, T.; Therrien, B.; Hendricks, D. T.; Guzgay, H.; Smith, G. S. Mono- and dinuclear ( $\eta 6$-arene) ruthenium(II) benzaldehyde thiosemicarbazone complexes: Synthesis, characterization and cytotoxicity. Inorg. Chem. Commun. 2011, 14, 956.

(43) Su, W.; Tang, Z.; Li, P.; Wang, G.; Xiao, Q.; Li, Y.; Huang, S.; Gu, Y.; Lai, Z.; Zhang, Y. New dinuclear ruthenium arene complexes containing thiosemicarbazone ligands: synthesis, structure and cytotoxic studies. Dalton Trans. 2016, 45, 19329.

(44) Mendoza-Ferri, M. G.; Hartinger, C. G.; Eichinger, R. E.; Stolyarova, N.; Severin, K.; Jakupec, M. A.; Nazarov, A. A.; Keppler, B. $\mathrm{K}$. Influence of the Spacer Length on the in Vitro Anticancer Activity of Dinuclear Ruthenium-Arene Compounds. Organometallics 2008, 27, 2405.

(45) Mendoza-Ferri, M. G.; Hartinger, C. G.; Mendoza, M. A.; Groessl, M.; Egger, A. E.; Eichinger, R. E.; Mangrum, J. B.; Farrell, N. P.; Maruszak, M.; Bednarski, P. J.; Klein, F.; Jakupec, M. A.; Nazarov, A. A.; Severin, K.; Keppler, B. K. Transferring the Concept of Multinuclearity to Ruthenium Complexes for Improvement of Anticancer Activity. J. Med. Chem. 2009, 52, 916.

(46) Nováková, O.; Nazarov, A. A.; Hartinger, C. G.; Keppler, B. K.; Brabec, V. DNA interactions of dinuclear Ru II arene antitumor complexes in cell-free media. Biochem. Pharmacol. 2009, 77, 364.

(47) Chen, H.; Parkinson, J. A.; Nováková, O.; Bella, J.; Wang, F.; Dawson, A.; Gould, R.; Parsons, S.; Brabec, V.; Sadler, P. J. Induced-fit recognition of DNA by organometallic complexes with dynamic stereogenic centers. Proc. Natl. Acad. Sci. U. S. A. 2003, 100, 14623.

(48) Pettinari, R.; Marchetti, F.; Pettinari, C.; Petrini, A.; Skelton, B. W.; White, A. H.; Bonfili, L.; Cuccioloni, M.; Eleuteri, A. M. Dinuclear ( 6-arene) ruthenium(II) acylpyrazolone complexes: Synthesis, characterization and cytotoxicity. J. Organomet. Chem. 2015, 791, 1.

(49) Eichhorn, T.; Hey-Hawkins, E.; Maksimović-Ivanić, D.; Mojić, M.; Schmidt, J.; Mijatović, S.; Schmidt, H.; Kaluđerović, G. N. Binuclear dichlorido( $\eta$ 6-p-cymene)ruthenium(II) complexes with bis(nicotinate)- and bis(isonicotinate)-polyethylene glycol ester ligands. Appl. Organomet. Chem. 2015, 29, 20.

(50) Murray, B. S.; Menin, L.; Scopelliti, R.; Dyson, P. J. Conformational control of anticancer activity: the application of arene-linked dinuclear ruthenium(ii) organometallics. Chem. Sci. 2014, $5,2536$.

(51) Murray, B. S.; Crot, S.; Siankevich, S.; Dyson, P. J. Potential of Cycloaddition Reactions To Generate Cytotoxic Metal Drugs In Vitro. Inorg. Chem. 2014, 53, 9315.

(52) Berners-Price, S. J.; Mirabelli, C. K.; Johnson, R. K.; Mattern, M. R.; McCabe, F. L.; Faucette, L. F.; Sung, C. M.; Mong, S. M.; Sadler, P. J.; Crooke, S. T. In vivo antitumor activity and in vitro cytotoxic properties of bis [1, 2-bis (diphenylphosphino) ethane] gold (I) chloride. Cancer Res. 1986, 46, 5486.

(53) Mirabelli, C. K.; Jensen, B. D.; Mattern, M. R.; Sung, C. M.; Mong, S. M.; Hill, D. T.; Dean, S. W.; Schein, P. S.; Johnson, R. K.; Crooke, S. T. Cellular pharmacology of $\mu$-[1,2-bis(diphenylphosphino)ethane] bis[(1-thio-beta-D-gluco pyranosato-S) gold(I)]: a novel antitumor agent. Anticancer Drug Des. 1986, 1, 223.

(54) Humphreys, A. S.; Filipovska, A.; Berners-Price, S. J.; Koutsantonis, G. A.; Skelton, B. W.; White, A. H. Gold(i) chloride adducts of 1,3-bis(di-2-pyridylphosphino)propane: synthesis, structural studies and antitumour activity. Dalton Trans. 2007, 43, 4943.

(55) Liu, J. J.; Galettis, P.; Farr, A.; Maharaj, L.; Samarasinha, H.; McGechan, A. C.; Baguley, B. C.; Bowen, R. J.; Berners-Price, S. J.; McKeage, M. J. In vitro antitumour and hepatotoxicity profiles of 
$\mathrm{Au}(\mathrm{I})$ and $\mathrm{Ag}(\mathrm{I})$ bidentate pyridyl phosphine complexes and relationships to cellular uptake. J. Inorg. Biochem. 2008, 102, 303.

(56) Tian, S.; Siu, F. M.; Kui, S. C. F.; Lok, C. N.; Che, C. M. Anticancer gold (i)-phosphine complexes as potent autophagyinducing agents. Chem. Commun. 2011, 47, 9318.

(57) Li, B. B.; Jia, Y. X.; Zhu, P. C.; Chew, R. J.; Li, Y.; Tan, N. S.; Leung, P. H. Highly selective anti-cancer properties of ester functionalized enantiopure dinuclear gold(I)-diphosphine. Eur. J. Med. Chem. 2015, 98, 250.

(58) Atrián-Blasco, E.; Gascón, S.; Rodríguez-Yoldi, M. J.; Laguna, M.; Cerrada, E. Synthesis of Gold(I) Derivatives Bearing Alkylated 1,3,5-Triaza-7-phosphaadamantane as Selective Anticancer Metallodrugs. Eur. J. Inorg. Chem. 2016, 17, 2791.

(59) Mirabelli, C. K.; Hill, D. T.; Faucette, L. F.; McCabe, F. L.; Girard, G. R.; Bryan, D. B.; Sutton, B. M.; Barus, J. O. L.; Crooke, S. T.; Johnson, R. K. Antitumor activity of bis (diphenylphosphino) alkanes, their gold (I) coordination complexes, and related compounds. J. Med. Chem. 1987, 30, 2181.

(60) Meyer, A.; Gutiérrez, A.; Ott, I.; Rodríguez, L. Phosphinebridged dinuclear gold (I) alkynyl complexes: Thioredoxin reductase inhibition and cytotoxicity. Inorg. Chim. Acta 2013, 398, 72.

(61) Barreiro, E.; Casas, J. S.; Couce, M. D.; Sánchez, A.; SánchezGonzalez, A.; Sordo, J.; Vázquez-López, E. M. Mono and dinuclear phosphinegold(I) sulfanylcarboxylates: Influence of nuclearity and substitution of PPh3 for PEt3 on cytotoxicity. J. Inorg. Biochem. 2014, $138,89$.

(62) Zou, T.; Lum, C. T.; Lok, C.; To, W.; Low, K.; Che, C. A Binuclear Gold(I) Complex with Mixed Bridging Diphosphine and Bis(N-Heterocyclic Carbene) Ligands Shows Favorable Thiol Reactivity and Inhibits Tumor Growth and Angiogenesis In Vivo. Angew. Chem., Int. Ed. 2014, 53, 5810.

(63) Barnard, P. J.; Wedlock, L. E.; Baker, M. V.; Berners-Price, S. J.; Joyce, D. A.; Skelton, B. W.; Steer, J. H. Luminescence Studies of the Intracellular Distribution of a Dinuclear Gold(I) N-Heterocyclic Carbene Complex. Angew. Chem., Int. Ed. 2006, 45, 5966.

(64) Rieb, J.; Dominelli, B.; Mayer, D.; Jandl, C.; Drechsel, J.; Heydenreuter, W.; Sieber, S. A.; Kuhn, F. E. Influence of Wing-tip Substituents and Reaction Conditions on Structure, Properties and Cytotoxicity of $\mathrm{Ag}(\mathrm{I})$ - and $\mathrm{Au}(\mathrm{I})$-bis(NHC) Complexes. Dalton Trans. 2017, 46, 2722.

(65) De Nisi, A.; Bergamini, C.; Leonzio, M.; Sartor, G.; Fato, R.; Naldi, M.; Monari, M.; Calonghi, N.; Bandini, M. Synthesis, cytotoxicity and anti-cancer activity of new alkynyl-gold (I) complexes. Dalton Trans. 2016, 45, 1546.

(66) Vergara, E.; Cerrada, E.; Casini, A.; Zava, O.; Laguna, M.; Dyson, P. J. Antiproliferative activity of gold (i) alkyne complexes containing water-soluble phosphane ligands. Organometallics 2010, 29, 2596.

(67) Chui, C. H.; Wong, R. S. M.; Gambari, R.; Cheng, G. Y. M.; Yuen, M. C. W.; Chan, K. W.; Tong, S. W.; Lau, F. Y.; Lai, P. B. S.; Lam, K. H.; Ho, C. L.; Kan, C. W.; Leung, K. S. Y.; Wong, W. Y. Antitumor activity of diethynylfluorene derivatives of gold (I). Bioorg. Med. Chem. 2009, 17, 7872.

(68) Altaf, M.; Monim-ul-Mehboob, M.; Isab, A. A.; Dhuna, V.; Bhatia, G.; Dhuna, K.; Altuwaijri, S. The synthesis, spectroscopic characterization and anticancer activity of new mono and binuclear phosphanegold(i) dithiocarbamate complexes. New J. Chem. 2015, 39, 377.

(69) Sun, R. W. Y.; Zhang, M.; Li, D.; Zhang, Z. F.; Cai, H.; Li, M.; Xian, Y. J.; Ng, S. W.; Wong, A. S. T. Dinuclear Gold(I) Pyrrolidinedithiocarbamato Complex: Cytotoxic and Antimigratory Activities on Cancer Cells and the Use of Metal-Organic Framework. Chem. - Eur. J. 2015, 21, 18534.

(70) Uson, R.; Laguna, A. Polyaryl Derivatives of gold(I), silver(I) and gold(III); Elsevier: Amsterdam, The Netherlands, 1986; Vol. 3, p 322.

(71) Vergara, E.; Miranda, S.; Mohr, F.; Cerrada, E.; Tiekink, E. R. T.; Romero, P.; Mendía, A.; Laguna, M. Gold (I) and Palladium (II) Thiolato Complexes Containing Water-Soluble Phosphane Ligands. Eur. J. Inorg. Chem. 2007, 18, 2926.
(72) Gutiérrez, A.; Gracia-Fleta, L.; Marzo, I.; Cativiela, C.; Laguna, A.; Gimeno, M. C. Gold (I) thiolates containing amino acid moieties. Cytotoxicity and structure-activity relationship studies. Dalton Trans. 2014, 43, 17054.

(73) Allardyce, C. S.; Dyson, P. J.; Ellis, D. J.; Heath, S. L. [Ru ( $76-$-pcymene) $\mathrm{Cl} 2$ (pta)] $(\mathrm{pta}=1,3$, 5-triaza-7-phosphatricyclo-[3.3. 1.1] decane): a water soluble compound that exhibits $\mathrm{pH}$ dependent DNA binding providing selectivity for diseased cells. Chem. Commun. 2001, 15, 1396.

(74) Hill, D. T.; Sutton, B. M. (2, 3, 4, 6-TETRA-O-ACETYL-1T H I O - B E T A - D - G L U C O P Y R A N O S A T O - S ) (TRIETHYLPHOSPHINE) GOLD, C20H34AUO9PS. Cryst. Struct. Commun. 1980, 9, 679.

(75) Tetko, I. V.; Gasteiger, J.; Todeschini, R.; Mauri, A.; Livingstone, D.; Ertl, P.; Palyulin, V. A.; Radchenko, E. V.; Zefirov, N. S.; Makarenko, A. S.; Tanchuk, V. Y.; Prokopenko, V. V. Virtual Computational Chemistry Laboratory - Design and Description. J. Comput.-Aided Mol. Des. 2005, 19, 453.

(76) Tetko, I. V.; Bruneau, P. Application of ALOGPS to predict 1octanol/water distribution coefficients, $\log \mathrm{P}$, and $\log \mathrm{D}$, of AstraZeneca in-house database. J. Pharm. Sci. 2004, 93, 3103.

(77) Sangster, J. Octanol-water partition coeficients of simple organic compounds. J. Phys. Chem. Ref. Data 1989, 18, 1111.

(78) Tönnemann, J.; Risse, J.; Grote, Z.; Scopelliti, R.; Severin, K. Efficient and Rapid Synthesis of Chlorido-Bridged Half-Sandwich Complexes of Ruthenium, Rhodium, and Iridium by Microwave Heating. Eur. J. Inorg. Chem. 2013, 26, 4558.

(79) Duisenberg, A. J. M.; Kroon-Batenburg, L. M. J.; Schreurs, A. M. M. An intensity evaluation method: EVAL-14. J. Appl. Crystallogr. 2003, 36, 220.

(80) Blessing, R. H. An empirical correction for absorption anisotropy. Acta Crystallogr., Sect. A: Found. Crystallogr. 1995, 51, 33.

(81) Sheldrick, G. M. A short history of SHELX. Acta Crystallogr., Sect. A: Found. Crystallogr. 2008, 64, 112.

(82) Sheldrick, G. M. Crystal structure refinement with SHELXL. Acta Crystallogr., Sect. C: Struct. Chem. 2015, 71, 3.

(83) Mosmann, T. Rapid colormetric assay for cellular growth and survival: Application to proliferation and cytotoxicity assays. $J$. Immunol. Methods 1983, 65, 55. 